\title{
KNOTS AND DISTRIBUTIVE HOMOLOGY: FROM ARC COLORINGS TO YANG-BAXTER HOMOLOGY
}

\author{
JÓZEF H. PRZYTYCKI
}

\section{Contents}

1. Introduction

1.1. Invariants of arc colorings

2. Monoid of binary operations 6

3. Homology of magmas $\quad 10$

3.1. Homology of abstract simplicial complexes 10

3.2. Degenerate subcomplex $\quad 14$

3.3. Bicomplex for a degenerate subcomplex $C_{n}^{D} \quad 17$

3.4. Homology with coefficients in a $k$-Mod functor $\quad 17$

4. Group homology of a semigroup 21

5. Hochschild homology of a semigroup and an algebra 24

6. Homology of distributive structures 26

6.1. One-term distributive homology 27

6.2. Multi-term distributive homology 28

7. Bloh-Leibniz-Loday algebra 31

8. Semigroup extensions and shelf extensions 34

8.1. Extensions in right distributive case 37

8.2. Extensions in entropic case 40

9. Degeneracy for a weak and very weak simplicial module 41

10. Degeneracy for a weak simplicial module 42

10.1. Right filtration of degenerate distributive elements 45

10.2. Integration maps $\hat{u}_{i}: \hat{F}_{n}^{p} \rightarrow \hat{F}_{n}^{p-1} \quad 4$

10.3. Weak simplicial modules with integration 47

11. Degeneracy for a very weak simplicial module 47

\footnotetext{
${ }^{1}$ This paper has its roots in two series of talks I gave: in Russia (Lomonosov Moscow State University, May 29-June 1, 2012), where the visualization in Figure 6.2 was observed, Korea (TAPU Workshop on Knot Theory, July 23-27, 2012), and in a talk at Oberwolfach Conference (June 3-9, 2012). The short version of this paper was published in Oberwolfach Proceedings [Prz-6]. While I keep novelty of the talks (many new ideas were crystallized then), I added a lot of supporting material so the paper is mostly self-sufficient. I also kept, to some extent, the structure of talks; it may lead to some repetitions but I hope it is useful for a reader.
} 
11.1. Introduction to t-simplicial objects 50

12. From distributive homology to Yang-Baxter homology 50

12.1. Graphical visualization of Yang-Baxter face maps 52

13. Geometric realization of simplicial and cubic sets $\quad 53$

13.1. Geometric realization of a (pre)cubic set 54

14. Higher dimensional knot theory $M^{n} \rightarrow \mathbb{R}^{n+2} \quad 57$

15. Acknowledgements 58

References $\quad 58$

\section{INTRODUCTION}

While homology theory of associative structures, such as groups and rings, has been extensively studied in the past beginning with the work of Hurewicz, Hopf, Eilenberg, and Hochschild, the non-associative structures, such as racks or quandles, were neglected until recently. The distributive structures 1 have been studied for a long time and already C.S. Peirce (1839-1914) in 1880 [Peir emphasized the importance of (right) self-distributivity in algebraic structures, and his friend E. Schröder, Schr] gave an example of a three element magma $(X ; *)$ which is not associative 2 (compare Section 2).

However, homology for such universal algebras was introduced only between 1990 and 1995 by Fenn, Rourke, and Sanderson [FRS-1, FRS-2, FRS-3, Fenn . We develop theory in the historical context and propose a general framework to study homology of distributive structures. We outline potential relations to Khovanov homology and categorification, via Yang-Baxter operators. We use here the fact that Yang-Baxter equation can be thought of as a generalization of self-distributivity.

1.1. Invariants of arc colorings. Consider a link diagram $D$, say , and a finite set $X$. We may define a diagram invariant to be the

\footnotetext{
${ }^{1}$ The word distributivity was coined in 1814 by French mathematician Francois Servois (1767-1847).

${ }^{2}$ The example Schröder (1841-1902) gave is

\begin{tabular}{l|lll}
$*$ & 0 & 1 & 2 \\
\hline 0 & 0 & 2 & 1 \\
1 & 2 & 1 & 0 \\
2 & 1 & 0 & 2
\end{tabular}

and we named elements of the magma by 0,1 and 2 as this example is the base for Fox three colorings of links (developed about 1956) [C-F, Cro, Prz-3, and the operation can be written as $x * y=2 y-x$ modulo 3 ; it happen to be self-distributive from both sides, that is $(x * y) * z=(x * z) *(y * z)$ and $x *(y * z)=(x * y) *(x * z)$ (see Example 2.3(5)).
} 
number of colorings of arcs 3 of $D$ by elements of $X, \operatorname{col}_{X}(D)$. Even such a naive definition leads to a link invariant $\operatorname{col}_{X}(L)=\min _{D \in L} \operatorname{col}_{X}(D)$, where $D \in L$ means that $D$ is a diagram of $L 4$ More sensible approach would start with a magma $(X ; *)$, that is a set with binary operation, and with the coloring convention of Figure 1.1.

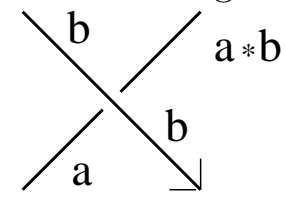

Figure 1.1; convention for a magma coloring of a crossing

Again, let for a finite $X, \operatorname{col}_{(X ; *)}(D)$ denote the number of colorings of arcs of $D$ by elements of $X$, according to the convention given in Figure 1.1, at every crossing. We can define an oriented link invariant by considering $\operatorname{col}_{(X ; *)}(L)=\min _{D \in L} \operatorname{col}_{(X ; *)}(D)$; Alternatively, we can minimize $\operatorname{col}_{(X ; *)}(L)$ over minimal crossing diagrams of $L$ only. Such an invariant would be very difficult to compute so it is better to look for properties of $(X ; *)$ so that $\operatorname{col}_{(X ; *)}(D)$ is invariant under Reidemeister moves: $R_{1}(\leftrightarrow \rho)$ gives idempotent relation $a * a=a$ 占 $R_{2}$ ()$(-\zeta)$ forces $*$ to be an invertible operation, and the third move illustrated in detail in Figure 1.2 below, forces on $*$ a right self-distributivity $(a * b) * c=(a * c) *(b * c)$.
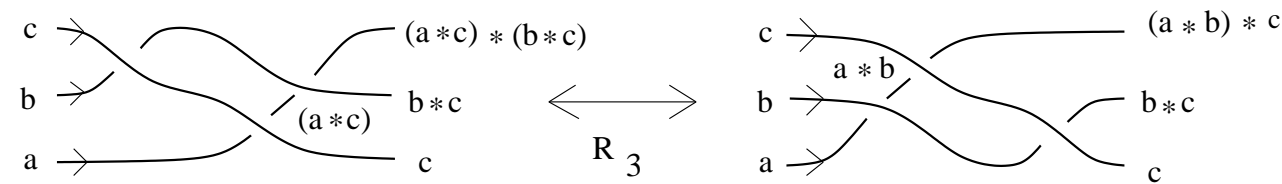

Figure 1.2; magma coloring of a third Reidemeister move and distributivity

\footnotetext{
${ }^{3}$ We use the term arc for a part of the diagram from an undercrossing to the next undercrossing (including possibility of a trivial component), and the term semi-arc for a part of the diagram from a crossing to the next crossing. Thus, for example, a standard trefoil knot diagram has three arcs and six semi-arcs.

${ }^{4}$ One can say that it is nonsense but an invariant is nontrivial: $\operatorname{col}_{X}(L)=$ $|X|^{\operatorname{cr}(L)+t(L)}$, where $\operatorname{cr}(L)$ is the crossing number of $L$ and $t(L)$ the number of trivial components in $L$. This is the case as for a knot diagram $D$ with at least one crossing the number of arcs equals to the number of crossings.

${ }^{5}$ The names idempotent (same power) and nilpotent (zero power) were introduced in 1870 by Benjamin Peirce [Pei], page 20.
} 
The magma $(X ; *)$ satisfying all three conditions is called a quandle, the last two - a rack, and only the last condition - a shelf or RDS (right distributive system). Thus, if $(X ; *)$ is a quandle then $\operatorname{col}_{(X ; *)}(D)$ (which we denote from now on succinctly $\operatorname{col}_{X}(D)$ ) is a link invariant. We also use the notation $\operatorname{Col}_{X}(D)$ for the set of $X$-colorings of $D$, thus $\operatorname{col}_{X}(D)=\left|\operatorname{Col}_{X}(D)\right|$. We also can try more generally to color semi-arcs of a diagram by elements of $X$ and declare for each colored crossing a weight of the crossing. This approach leads to state sums and Yang-Baxter operators (see Section [6] and Figures 6.2, 12.1, and 12.3).

We also can do more with distributive magmas (after S.Carter, S.Kamada, and M.Saito [CKS]; compare also M.Greene thesis [Gr]). We can sum over all crossings the pairs $\pm(a, b)$ according to the convention
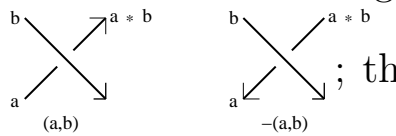
the investigation of invariance of $\sum \pm(a, b)$ under

Reidemeister moves was a hint toward construction of (co)homology of quandles.

We also encounter right distributivity by asking the following question: for a given coloring $\phi \in \operatorname{Col}_{X}(D)$ by a magma $(X ; *)$, and an element $x \in X$ is coloring $\phi * x$ also a magma coloring? If, as before, coloring is given by $a, b$, and $c=a * b$ then the new coloring of a crossing is given by $a * x, b * x$, and $c * x$. For a magma coloring we need $(a * b) * x=(a * x) *(b * x)$ which is exactly right self-distributivity. To put it on a more solid footing, we observe that the map $*_{x}: X \rightarrow X$ with $*_{x}(a)=a * x$ is a magma homomorphism: $*_{x}(a * b)=(a * b) * x=(a * x) *(b * x)=*_{x}(a) * *_{x}(b)$. For any magma homomorphism $g: X \rightarrow X$ if $f: \operatorname{arcs}(D) \rightarrow X$ is a magma coloring then $g f$ defined by $(g f)(\operatorname{arc})=g(f(\operatorname{arc}))$ is a magma coloring of $D$. These are classical observations thus it is interesting to notice the slightly more general fact concerning the following question:

Let $\left(X ; *_{1}\right)$ be a magma and $f$ and $g$ two $\left(X ; *_{1}\right)$ magma colorings of a diagram $D$. Find the magma operation $*_{2}$ so that $f *_{2} g$ is also a $\left(X ; *_{1}\right)$ magma coloring. The question reduces to the previous one if $g$ is a trivial (i.e. constant) coloring and $*_{1}=*_{2}$. The nice condition which answers the question was first discussed by M.Niebrzydowski at his talk at Knots in Washington XXXV conference in December of 2012 [Nieb] (compare also [C-N]).

Lemma 1.1. Let $f, g: \operatorname{arcs}(D) \rightarrow X$ be two colorings of a diagram $D$ by $\left(X ; *_{1}\right)$ (that is $f, g \in \operatorname{Col}_{\left(X ; *_{1}\right)}(D)$ ). Then $f *_{2} g$ where $*_{2}$ is another binary operation on $X$ and $\left(f *_{2} g\right)(\operatorname{arc})=f(\operatorname{arc}) *_{2} g(\operatorname{arc})$, is 
an $\left(X ; *_{1}\right)$ coloring of $X$ if operations $*_{1}$, and $*_{2}$ are entropic one with respect to the other, that is: $\left(a *_{1} b\right) *_{2}\left(c *_{1} d\right)=\left(a *_{2} c\right) *_{1}\left(b *_{2} d\right)$.

Proof. For every crossing with initial under-arc $a$ and over-arc $b$ we need

$$
\left(f(a) *_{2} g(a)\right) *_{1}\left(f(b) *_{2} g(b)\right)=\left(f(a) *_{1} f(b)\right) *_{2}\left(g(a) *_{1} g(b)\right)
$$

which is exactly the entropic condition in Figure 1.3 (compare Subsection 8.2).

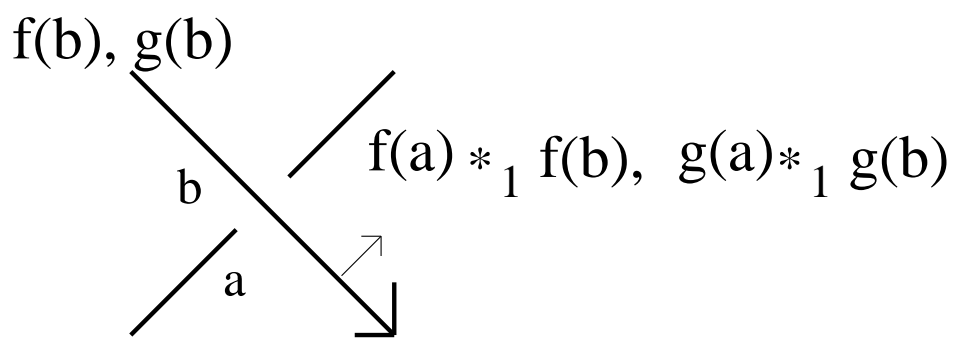

\section{$\mathrm{f}(\mathrm{a}), \mathrm{g}(\mathrm{a}) \quad$ colorings by $\mathrm{f}$ and $\mathrm{g}$}

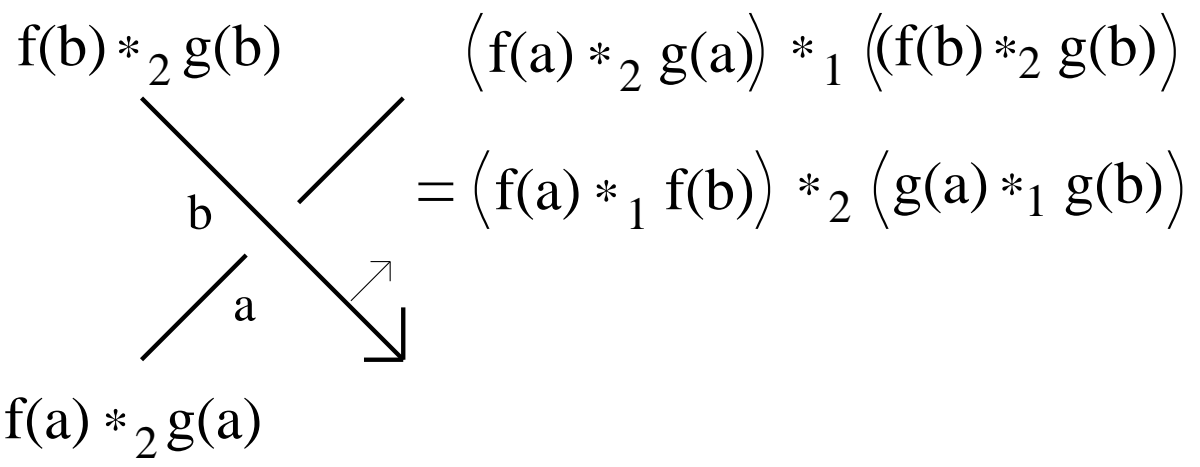

Figure 1.3; Entropy condition for composition of $f$ and $g: f *_{2} g$

Notice that if $g$ is a trivial coloring, say $g(\operatorname{arc})=x$ for any arc then any crossing forces $x *_{1} x=x$ and the entropic equation reduces to $\left(f(a) *_{2} x\right) *_{1}\left(f(b) *_{2} x\right)=\left(f(a) *_{1} f(b)\right) *_{2}\left(x *_{1} x\right)=\left(f(a) *_{1} f(b)\right) *_{2} x$ (right distributivity). For use of entropic magmas in Knot Theory, see [N-P-4, Prz-1, Prz-2, Prz-8, P-T-1, P-T-2, Si]; compare also Proposition 2.6 .

We introduce now a monoid of binary operations and show that distributivity can be studied in the context of this monoid. Then we compare homology for associative structures (semigroups) with that 
for distributive structures (shelves). We also compare extensions in associative and distributive cases.

The paper is planned as a continuation of pioneering essay [Prz-5] and for completeness we recall parts of the essay.

\section{MONOID OF BINARY OPERATIONS}

Let $X$ be a set and $*: X \times X \rightarrow X$ a binary operation. We call $(X ; *)$ a magm 6 . For any $b \in X$ the adjoint map $*_{b}: X \rightarrow X$, is defined by $*_{b}(a)=a * b$. Let $\operatorname{Bin}(X)$ be the set of all binary operations on $X$.

Proposition 2.1. Bin $(X)$ is a monoid (i.e. semigroup with identity) with the composition $*_{1} *_{2}$ given by $a *_{1} *_{2} b=\left(a *_{1} b\right) *_{2} b$, and the identity $*_{0}$ being the right trivial operation, that is, $a *_{0} b=a$ for any $a, b \in X$.

If $* \in \operatorname{Bin}(X)$ is invertible then $*^{-1}$ is usually denoted by $\bar{*}$. One should remark that $*_{0}$ is distributive with respect to any other operation, that is, $(a * b) *_{0} c=a * b=\left(a *_{0} c\right) *\left(b *_{0} c\right)$, and $\left(a *_{0} b\right) * c=$ $a * c=(a * c) *_{0}(b * c)$.

Definition 2.2. Let $(X ; *)$ be a magma, then:

(i) If $*$ is right self-distributive, that is, $(a * b) * c=(a * c) *(b * c)$, then $(X ; *)$ is called an $R D S$ (right distributive structure) or a shelf (the term coined by Alissa Crans and used in knot theory [Cr]).

(ii) If a shelf $(X ; *)$ satisfies the idempotent condition, $a * a=a$ for any $a \in X$, then it is called an RDI structure or right spindle, or just a spindle (again the term coined by Crans).

(iii) If a shelf $(X ; *)$ has $*$ invertible in Bin $(X)$ (equivalently $*_{b}$ is a bijection for any $b \in X$ ), then it is called a rack (the term wrack, like in "wrack and ruin", of J.H.Conway from 1959 [C-W], was modified to rack in $[\mathrm{F}-\mathrm{R}])$.

(iv) If a rack $(X ; *)$ satisfies the idempotent condition, then it is called a quandle (the term coined in Joyce's PhD thesis of 1979 Joy-1, Joy-2]). Axioms of a quandle were motivated by three Reidemeister moves (idempotent condition by the first move, invertibility by the second, and right self-distributivity by the third move).

\footnotetext{
${ }^{6}$ The term magma was used by J-P. Serre [Ser] and Bourbaki [Bou, replacing the older term groupoid which started to mean a category with every morphism invertible.
} 
(v) If a quandle $(X ; *)$ satisfies $* *=*_{0}$ (i.e. $\left.(a * b) * b=a\right)$ then it is called kei or an involutive quandle. The term kei (圭) was coined in a pioneering paper by M. Takasak in 1942 [Tak]

The main early example of a rack (and a quandle) was a group $G$ with a $*$ operation given by conjugation, that is, $a * b=b^{-1} a b$ (Conway thought of this as a "wrack" of a group). Another example, considered already in Conway-Wraith correspondence [C-W], is defined for any group with $a * b=b a^{-1} b$ and called by Joyce (after Bruck [Bru]) a core quandle. This example, for a group, $H$, abelian was already studied by Takasaki so we call it Takasaki kei (or quandle) and denote by $T(H)$ (in abelian notation we have $a * b=2 b-a$ ), compare [N-P-1]. $T\left(Z_{n}\right)$ is often called a dihedral quandle and denoted by $R_{n}$; it can be interpreted as composed of reflections of the dihedral group $D_{2 n}$ (we can mention that rack and quandle homology of $T\left(Z_{n}\right)$ for prime $n$ has been computed in [N-P-2, Cla, Nos]).

More general examples still starting from a group are given in Joyce paper Joy-2:

Example 2.3. Let $G$ be a group and $t: G \rightarrow G$ a group homomorphism then we have the following spindle structures on $G$ :

(1) $a *_{1} b=t\left(a b^{-1}\right) b$,

(2) $a *_{2} b=t\left(b^{-1} a\right) b$,

(3) If $t$ is invertible both examples give quandles where $\bar{*}_{1}$ and $\bar{*}_{2}$ are given by the formulas:

(i) $a \bar{*}_{1} b=t^{-1}\left(a b^{-1}\right) b$ thus $\bar{*}_{1}$ yielded by the automorphism $t$ is equal to $*_{1}$ yielded by the automorphism $t^{-1}$.

(ii) $a \bar{*}_{2} b=b t^{-1}\left(a b^{-1}\right)$, e.g. we check that

$\left(a *_{2} b\right) \bar{*}_{2} b=b t^{-1}\left(\left(t\left(b^{-1} a\right) b b^{-1}\right)=a\right.$. This example is related to the fundamental group of cyclic (branched) covers of $S^{3}$ along a link. Locally, at every crossing we have relations $C=\tau^{-1}\left(B^{-1} A\right) B$ and $A=B \tau\left(C B^{-1}\right)$, as illustrated in Figure 2.1 [Prz-3, Pr-Ro-1, DPT].

(4) If $G$ is an abelian group both examples lead to the same spindle called Alexander spindle (for $t$ invertible, Alexander quandle). In abelian notation we get $a * b=t a-t b+b=(1-t) b+t a$.

\footnotetext{
${ }^{7}$ Mituhisa Takasaki worked at Harbin Technical University, likely as an assistant to Kôshichi Toyoda. Both perished when Red Army entered Harbin in August 1945 .
} 
This two sided distributive structure was already considered in 1929 by C.Bursting and W.Mayen $[\mathrm{B}-\mathrm{M}]$.

(5) If $t=-1$ we get $a * b=2 b-a$ and this structure, as mentioned before, was the main example of Kei by Takasaki so we denote it by $T(G)$.

(6) $a *_{3} b=t\left(b a^{-1}\right) b$ with $t^{2}=t$. It is a quandle if and only if $t=I d$ in which case we get a quandle called the core quandle of $G$.

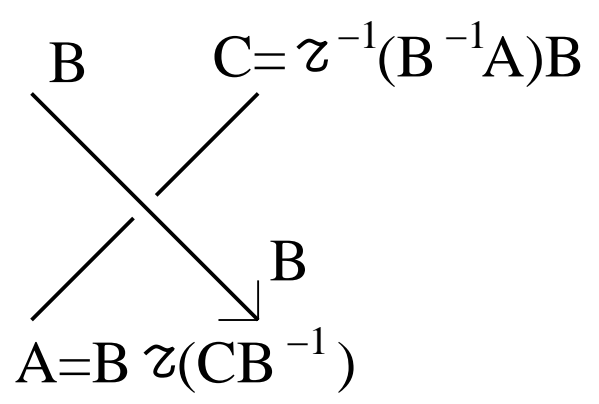

Figure 2.1; relations for cyclic covering; see Example 2.3(3)

Definition 2.2 describes properties of an individual magma $(X ; *)$. It is also useful to consider subsets or submonoids of $\operatorname{Bin}(X)$ satisfying the related conditions (compare [R-S, Mov, Deh-1, Prz-5].

Definition 2.4. We say that a subset $S \subset \operatorname{Bin}(X)$ is a distributive set if all pairs of elements $*_{\alpha}, *_{\beta} \in S$ are right distributive, that is, $\left(a *_{\alpha} b\right) *_{\beta} c=\left(a *_{\beta} c\right) *_{\alpha}\left(b *_{\beta} c\right)$ (we allow $\left.*_{\alpha}=*_{\beta}\right)$.

(i) The pair $(X ; S)$ is called a multi-shelf if $S$ is a distributive set. If $S$ is additionally a submonoid (resp. subgroup) of $\operatorname{Bin}(X)$, we say that it is a distributive monoid (resp. group).

(ii) If $S \subset \operatorname{Bin}(X)$ is a distributive set such that each * in $S$ satisfies the idempotent condition, we call $(X ; S)$ a multi-spindle.

\footnotetext{
${ }^{8}$ Celestyn Burstin (1888-1938) was born in Tarnopol, where he obtained "Matura" in 1907, he moved to Vienna where in 1911 he completed university. In 1929 he moved to Minsk where he was a member of the Belarusian National Academy of Sciences, and a Director of the Institute of Mathematics of the Academy. In December 1937, he was arrested on suspicion of activity as a spy for Poland and Austria. He died in October 1938, when interrogated in a prison in Minsk ("Minskaja Tjurma"); he was rehabilitated March 2, 1956 [Bur-1, Bur-M, Mal, Mio.

${ }^{9}$ Walter Mayer (1887-1948) is well known for Mayer-Vietoris sequence and for being assistant to A.Einstein at Institute for Advanced Study, Princeton [Isa].
} 
(iii) We say that $(X ; S)$ is a multi-rack if $S$ is a distributive set, and all elements of $S$ are invertible.

(iv) We say that $(X ; S)$ is a multi-quandle if $S$ is a distributive set, and elements of $S$ are invertible and satisfy the idempotent condition.

(v) We say that $(X ; S)$ is a multi-kei if it is a multi-quandle with $* *=*_{0}$ for any $* \in S$. Notice that if $*_{1}^{2}=*_{0}$ and $*_{2}^{2}=$ $*_{0}$ then $\left(*_{1} *_{2}\right)^{2}=*_{0}$; more generally if $*_{1}^{n}=*_{0}$ and $*_{2}^{n}=$ $*_{0}$ then $\left(*_{1} *_{2}\right)^{n}=*_{0}$. This follows from the fact that elements of a multi-quandle commute pairwise (this was observed by M.Jabtonowski [Prz-5]).

Proposition 2.5. [Prz-5]

(i) If $S$ is a distributive set and $* \in S$ is invertible, then $S \cup\{\bar{*}\}$ is also a distributive set.

(ii) If $S$ is a distributive set and $M(S)$ is the monoid generated by $S$ then $M(S)$ is a distributive monoid.

(iii) If $S$ is a distributive set of invertible operations and $G(S)$ is the group generated by $S$, then $G(S)$ is a distributive group.

We show, after G.Mezera [Mez, the fact that any group can be embedded in $\operatorname{Bin}(X)$ for some $X$, in particular the regular embedding of $G$ in $\operatorname{Bin}(G)$ is given by $g \rightarrow *_{g}$ with $a *_{g} b=a b^{-1} g b$ (compare [Lar] and Example X.3.15 of [Deh-2] where the operation $a *_{g} b=a b^{-1} g b$ is called a half-conjugacy). The expression $a b^{-1} g b$ was also discussed with respect to free rack by Fenn and Rourke (compare Remark 8.2).

Proposition 2.5 has its analogue for entropic magmas (that is magmas for which $(a * b) *(c * d)=(a * c) *(b * d))$. More precisely, we say that a subset $S \in \operatorname{Bin}(X)$ is an entropic set if for any $*_{\alpha}, *_{\beta} \in S$ we have the entropic condition:

$$
\left(a *_{\alpha} b\right) *_{\beta}\left(c *_{\alpha} d\right)=\left(a *_{\beta} c\right) *_{\alpha}\left(b *_{\beta} d\right) . \text { Then we have: }
$$

\section{Proposition 2.6. N-P-4}

(i) If $S$ is an entropic set and $* \in S$ is invertible, then $S \cup\{\bar{*}\}$ is also an entropic set.

(ii) If $S$ is an entropic set and $M(S)$ is the monoid generated by $S$ then $M(S)$ is an entropic monoid.

(iii) If $S$ is an entropic set of invertible operations and $G(S)$ is the group generated by $S$, then $G(S)$ is an entropic group.

In the next section we consider homology theory of various magmas, it is useful here to define, for any magma $(X ; *)$ a supporting structure which we call an $X$-set (it is an old concept for (semi)groups and for quandles it was first considered by S.Kamada). 
Definition 2.7. Let $(X ; *)$ a magma and $E$ a set. We say that $E$ is an $X$-set (or right $X$-set) if there is a function (right action) $*_{E}$ : $E \times X \rightarrow E$. In general we do not put any conditions on $*_{E}$ but if our magma satisfies some conditions (e.g. associativity or distributivity) then $*_{E}$ should satisfy some related conditions. In particular, we will look for a magma structure on $X \sqcup E$ having similar structure.

In the following few sections we discuss various homology theories for magmas (e.g. associative or distributive). In broad approach we follow [Prz-5] but we stress the use of $X$-sets in our definitions.

\section{Homology of MAgmas}

We survey in this section various homology theories, starting from homology of abstract simplicial complexes; then we extract (old and new) properties to define a presimplicial module and a (weak) simplicial module. Further we give two examples of homology for associative structures (semigroups), and, an important in knot theory, example of homology for right self-distributive structures (RDS or shelves). Later we go back to very general notion of homology of a small category with coefficient in a functor to $R$-Mod, and recall the notion of a geometric realization in the case of a (pre)simplicial set, and (pre)cubic set. Reader interested only in distributive homology can go directly to Section 6.

3.1. Homology of abstract simplicial complexes. Our goal is to introduce homology of distributive magmas but to keep a historical perspective we start with the standard (oriented and ordered) homology of abstract simplicial complexes as they provide the framework for all homology we consider.

Definition 3.1. The abstract simplicial complex $\mathcal{K}=(V, P)$ is a pair of sets where $V=V(\mathcal{K})$ is called a set of vertices and $P(\mathcal{K})=P \subset 2^{V}$, called the set of simplexes of $\mathcal{K}$ and it satisfies: elements of $P$ are finite subsets of $V$, include all one-element subsets, and if $s^{\prime} \subset s \in P$ then also $s^{\prime} \in P$ (that is a subsimplex of a simplex is a simplex) 10 simplex of $n+1$ vertices is called $n$-dimensional simplex, or succinctly, $n$-simplex (we write $s=\left\{v_{i_{0}}, v_{i_{1}}, \ldots, v_{i_{n}}\right\}$ ). We define $\operatorname{dim}(\mathcal{K})$ as the maximal dimension of a simplex in $\mathcal{K}$ (may be $\infty$ if there is no bound).

We consider, additionally, the category of abstract simplicial complexes with a class of objects composed of abstract simplicial complexes.

\footnotetext{
${ }^{10}$ Usually we do not allow $\emptyset$ as a simplex, but in some situations it is convenient to allow also an empty simplex, say of dimension -1 ; it will lead naturally to augmented chain complexes.
} 
$\operatorname{Mor}\left(K_{1}, K_{2}\right)$ is the set of maps from $V\left(K_{1}\right)$ to $V\left(K_{2}\right)$ which send a simplex to a simplex (that is if $f \in \operatorname{Mor}\left(K_{1}, K_{2}\right), s \in P\left(K_{1}\right)$ then $\left.f(s) \in P\left(K_{2}\right)\right)$.

We recall here three classical (equivalent) definitions of a homology of an abstract simplicial complex: ordered, normalized ordered, and oriented.

Definition 3.2. Recall that a chain complex $\mathcal{C}$ is a sequence of modules over a fixed ring $k$ (here always commutative with identity),

$$
\mathcal{C}: \quad \ldots \stackrel{\partial_{n+2}}{\longrightarrow} C_{n+1} \stackrel{\partial_{n+1}}{\longrightarrow} C_{n} \stackrel{\partial_{n}}{\longrightarrow} C_{n-1} \stackrel{\partial_{n-1}}{\longrightarrow} \ldots
$$

such that $\partial_{n} \partial_{n+1}=0$ (succinctly $\partial^{2}=0$ ). Thus we have $i m \partial_{n+1} \subset$ ker $\partial_{n}$, and we define homology $H_{n}(\mathcal{C})=$ ker $\partial_{n} /$ im $\partial_{n+1}$.

Now for an abstract simplicial complex $K=(V, P)$ one defines:

(I) (Ordered homology)

Consider a chain complex $\mathcal{C}^{\text {ord }}$ with $k$-modules $C_{n}^{\text {ord }}=C_{n}^{\text {Ord }}(\mathcal{C})$ a submodule of $k V^{n+1}$ generated by all sequences $\left(x_{0}, x_{1}, \ldots, x_{n}\right)$, allowing repetitions, such that the set $\left\{x_{0}, x_{1}, \ldots, x_{n}\right\}$ is a simplex in $P$ (possibly of dimension smaller from $n$ ). The boundary operation is given on the basis by:

$$
\partial\left(x_{0}, x_{1}, \ldots, x_{n}\right)=\sum_{i=0}^{n}(-1)^{i} d_{i}=\sum_{i=0}^{n}(-1)^{i}\left(x_{0}, \ldots, x_{i-1}, x_{i+1}, \ldots, x_{n}\right) .
$$

The ordered homology of $\mathcal{K}$ is defined

$$
H_{n}^{\text {ord }}(\mathcal{K}, k)=k e r \partial_{n} / \text { im } \partial_{n+1} .
$$

If $k=\mathbb{Z}$ we write $H_{n}^{\text {ord }}(\mathcal{K})$.

Notices, that the maps $d_{i}: C_{n}^{\text {ord }} \rightarrow C_{n-1}^{\text {ord }},(0 \leq i \leq n), d_{i}\left(x_{0}, x_{1}, \ldots, x_{n}\right)=$ $\left(x_{0}, \ldots, x_{i-1}, x_{i+1}, \ldots, x_{n}\right)$, called the face maps, satisfy:

(1) $d_{i} d_{j}=d_{j-1} d_{i}$ for any $i<j$.

The system $\left(C_{n}, d_{i}\right)$ satisfying the above equality is called a presimplicial modul 11 and if we limit ourselves to $\left(V^{n+1}, d_{i}\right)$ it is called a presimplicial set (compare Definition 3.3). The important basic observation is that if $\left(C_{n}, d_{i}\right)$ is a presimplicial module then $\left(C_{n}, \partial_{n}\right)$, for $\partial_{n}=\sum_{i=0}^{n}(-1)^{i} d_{i}$, is a chain complex.

Motivation for the boundary operation:

it is coming from the geometrical realization of an abstract simplicial complex as illustrated below (the general setting of geometric realization of a simplicial set is discussed in Section 13):

\footnotetext{
${ }^{11}$ The concept was introduced in 1950 by Eilenberg and Zilber under the name semi-simplicial complex, E-Z].
} 


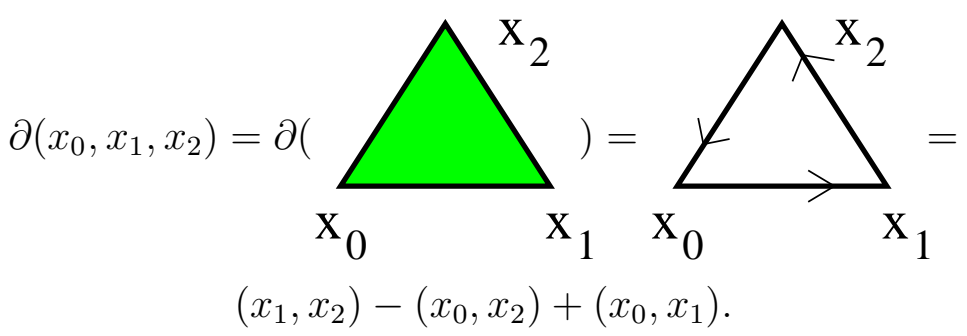

(II) (Normalized ordered homology).

The ordered chain complex allows degenerate simplexes (when vertices repeat, in particular neighboring vertices repeat). We define $i$-degeneracy maps $s_{i}: C_{n} \rightarrow C_{n+1}(0 \leq i \leq n)$ by $s_{i}\left(x_{0}, \ldots, x_{n}\right)=\left(x_{0}, \ldots, x_{i-1}, x_{i}, x_{i}, x_{i+1}, \ldots, x_{n}\right)$. We can check here easily that:

$$
\begin{gathered}
\text { (2) } s_{i} s_{j}=s_{j+1} s_{i}, \quad 0 \leq i \leq j \leq n, \\
\text { (3) } d_{i} s_{j}= \begin{cases}s_{j-1} d_{i} & \text { if } i<j \\
s_{j} d_{i-1} & \text { if } i>j+1\end{cases} \\
\text { (4) } d_{i} s_{i}=d_{i+1} s_{i}=I d_{C_{n}} .
\end{gathered}
$$

$\left(C_{n}, d_{i}, s_{i}\right)$ satisfying properties (1)-(4) is called a simplicial module. The notion was introduced by Eilenberg and Zilber in 1950 under the name of complete semi-simplicial complex [E-Z]. It is convenient to rephrase the definition so it can be used to any category:

Definition 3.3. Consider a category $\mathcal{C}$, the sequence of objects $X_{n}, n \geq 0$ and for any $n$ morphisms $d_{i}, s_{i}, 0 \leq i \leq n, d_{i} \in$ $\operatorname{Mor}\left(X_{n}, X_{n-1}\right)$, and $s_{i} \in \operatorname{Mor}\left(X_{n}, X_{n+1}\right)$. We call $\left(X_{n} ; d_{i}, s_{i}\right)$ a simplicial category (e.g. simplicial set, simplicial module, or simplicial space) if the following four conditions hold.

(1) $d_{i} d_{j}=d_{j-1} d_{i}$ for $i<j$,

(2) $s_{i} s_{j}=s_{j+1} s_{i}$ for $i \leq j$,

(3)

$$
\begin{aligned}
& s_{i} s_{j}=s_{j+1} s_{i}, \quad 0 \leq i \leq j \leq n, \\
& d_{i} s_{j}= \begin{cases}s_{j-1} d_{i} & \text { if } i<j \\
s_{j} d_{i-1} & \text { if } i>j+1\end{cases}
\end{aligned}
$$

(4) $d_{i} s_{i}=d_{i+1} s_{i}=I d_{X_{n}}$.

Eilenberg and Mac Lane proved in 1947 that the degenerate part of a presimplicial module is an acyclic chain complex (it has trivial homology) [E-M-1] (the prove was more specific but the method worked for all simplicial modules defined only 3 years 
later). We devote Subsection [3.2 to the proof, after [Lod-1], paying attention to which axioms of a simplicial module are used. In particular, axiom (4) cannot be ignored as the degenerate chain complex of quandles, which satisfies the property (4) only partially has often nontrivial homology.

Now back to normalized ordered homology:

Consider submodules $C_{n}^{D}(\mathcal{K}$ (named degenerated modules) and defined by

$$
C_{n}^{D}=\operatorname{span}\left(s_{0}\left(C_{n-1}\right), s_{1}\left(C_{n-1}\right), \ldots, s_{n-1}\left(C_{n-1}\right)\right) .
$$

One check that $\left(C_{n}^{D}, \partial_{n}\right)$ is a subchain complex of $C_{n}^{\text {ord }}(\mathcal{K})$. Details are given in Subsection 3.2. were it is also proved that this chain complex is acyclic. We have also quotient chain complex, called normalized ordered chain complex with $C_{n}^{N}(\mathcal{K})=$ $C_{n}(\mathcal{K}) / C_{n}^{D}(\mathcal{K})$. As homology groups of $C_{n}^{D}(\mathcal{K})$ are trivial we have isomorphism:

$$
H_{n}^{\text {ord }}(\mathcal{K}, k) \rightarrow H_{n}^{N}(\mathcal{K}, k) .
$$

(III) (Oriented homology). We can consider smaller chain complex giving the same homology of $\mathcal{K}$ by taking the quotient of $C^{N}(\mathcal{K})$ and considering only "oriented simplexes". Formally, let $\bar{C}_{n}(\mathcal{K})$ be a submodule of $C^{N}(\mathcal{K})$ generated by "transposition symmetrizers" $\left(x_{0}, \ldots x_{i-1}, x_{i}, x_{i+1}, x_{i+2}, \ldots, x_{n}\right)+\left(x_{0}, \ldots x_{i-1}, x_{i+1}, x_{i}, x_{i+2}, \ldots, x_{n}\right)$. One checks directly that $\left(\bar{C}_{n}(\mathcal{K}), \partial_{n}\right)$ is a subchain complex of $C^{N}(\mathcal{K})$. The oriented chain complex is the quotient: $C^{\text {ori }}(\mathcal{K})=$ $C^{N}(\mathcal{K}) / \bar{C}_{n}((\mathcal{K})$. It require some effort to prove that the quotient map $f: C^{N}(\mathcal{K}) \rightarrow C^{\text {ori }}(\mathcal{K})$ is a chain equivalence and thus $f_{*}: H^{N}(\mathcal{K}) \rightarrow H^{\text {ori }}(\mathcal{K})$ is is an isomorphism of homology modules. From this we conclude that all three definitions, ordered, normalized ordered, and oriented of homology of an abstract simplicial complex give the same result.

To have more concrete view of oriented chain complex and oriented homology we order vertices $V$ of $\mathcal{K}$ and interpret the chain group $C_{n}^{\text {ori }}(\mathcal{C})$ as a subgroup of $Z V^{n+1}$ freely generated by $n$-dimensional simplexes, $\left(x_{0}, x_{1}, \ldots, x_{n}\right)$ (we assume that $x_{0}<$ $x_{1}<\ldots<x_{n}$ in our ordering). In essence, with ordering, we are able to choose representatives of equivalence classes in $C_{n}^{\text {ori }}(\mathcal{K})$ and the boundary operation

$$
\partial\left(x_{0}, x_{1}, \ldots, x_{n}\right)=\sum_{i=0}^{n}(-1)^{i} d_{i} \text { where }
$$




$$
d_{i}\left(x_{0}, x_{1}, \ldots, x_{n}\right)=\left(x_{0}, \ldots, x_{i-1}, x_{i+1}, \ldots, x_{n}\right),
$$

preserves our choice so with given ordering we have a split chain map $g: C_{n}^{\text {ori }}(\mathcal{K}) \rightarrow C_{n}^{N}(\mathcal{K})$. The quotient map, with our ordering can be written as $f_{n}\left(x_{0}, \ldots, x_{n}\right)=(-1)^{|\pi|}\left(x_{0}^{\prime}, \ldots, x_{n}^{\prime}\right)$ where $\pi \in S_{n+1}$ is the permutation such that $x_{i}^{\prime}=x_{\pi(i)}$ and $x_{i}^{\prime}<x_{i+1}^{\prime}$ (if $x_{i}=x_{j}$ for some $i \neq j$ then we put $f\left(x_{0}, \ldots, x_{n}\right)=0$ ). Immediately, we have $f_{n} g_{n}=I d_{C_{n}^{\text {ori }}(\mathcal{K})}$. The proof that $g f$ is chain homotopic to identity on $C_{n}^{N}(\mathcal{K})$ (and so $f$ is chain equivalence) requires more effort 12

3.2. Degenerate subcomplex. Consider a presimplicial module $\left(C_{n}, d_{i}\right)$ with degenerate maps $s_{i}$. We define degenerate modules

$$
C_{n}^{D}=\operatorname{span}\left(s_{0}\left(C_{n-1}\right), s_{1}\left(C_{n-1}\right), \ldots, s_{n-1}\left(C_{n-1}\right)\right) .
$$

We check which conditions are needed so that $\left(C_{n}^{D}, d_{i}\right)$ is a sub-presimplicial module of $\left(C_{n}, d_{i}\right)$. We have:

$$
\begin{aligned}
\partial_{n} s_{p} & =\sum_{i=0}^{n+1}(-1)^{i} d_{i} s_{p}=\sum_{i=0}^{p-1}(-1)^{i} d_{i} s_{p}+(-1)^{p}\left(d_{p} s_{p}-d_{p-1} s_{p}+\sum_{i=p+2}^{n+1}(-1)^{i} d_{i} s_{p} \stackrel{(3)}{=}\right. \\
& \sum_{i=0}^{p-1}(-1)^{i} s_{p-1} d_{i}+(-1)^{p}\left(d_{p} s_{p}-d_{p+1} s_{p}+\sum_{i=p+2}^{n+1}(-1)^{i} s_{p} d_{i-1} \stackrel{\left(4^{\prime}\right)}{=}\right.
\end{aligned}
$$

\footnotetext{
${ }^{12}$ The standard Eilenberg-Mac Lane proof uses acyclic modules method E-M-2, $\mathrm{Spa}$, however in our case one can give shorter proof (the idea is still that of Eilenberg-Mac Lane): consider the chain map $f: \mathcal{C}^{N} \rightarrow \mathcal{C}^{\text {ori }}$ given by $f_{n}\left(x_{0}, \ldots, x_{n}\right)=(-1)^{|\pi|}\left(x_{0}^{\prime}, \ldots, x_{n}^{\prime}\right)$ where $\pi \in S_{n+1}$ is the permutation such that $x_{i}^{\prime}=x_{\pi(i)}$ and $x_{i}^{\prime}<x_{i+1}^{\prime}$ (if $x_{i}=x_{j}$ for some $i \neq j$ then we put $f\left(x_{0}, \ldots, x_{n}\right)=0$ ). The map $g: \mathcal{C}^{\text {ori }} \rightarrow \mathcal{C}^{N}$ is defined to be embedding; therefore $f g=I d_{\mathcal{C}}$. We show that $g f$ induces identity on homology of $\mathcal{C}^{N}$. We construct a chain homotopy between $g f$ and the identity inductively, starting from $F_{0}=0$. The main ingredient of the proof is the fact that for a simplex $s=\left(x_{0}, \ldots, x_{n}\right)$ the subchain complex $\bar{s}=\left(s, 2^{s}\right)$ of $\mathcal{C}^{N}$ is acyclic $\left(H_{n}^{N}(\bar{s})=0\right.$ for $n>0$ and $\left.H_{0}^{N}(\bar{s})=k\right)$.
}

Step n: assume that $F_{n-1}, \ldots, F_{0}$ are already constructed and we construct a map $F_{n}: C_{n}^{N} \rightarrow C_{n+1}^{N}$ such that $\partial_{n+1} F_{n}=-F_{n-1} \partial_{n}+I d-g f$.

We compute: that

$$
\begin{gathered}
\partial_{n}\left(-F_{n-1} \partial_{n}+I d-g f\right)=-\left(\partial_{n} F_{n-1}\right) \partial_{n}+\partial_{n}-\partial_{n}(g f)= \\
F_{n-2} \partial_{n-1} \partial_{n}-\partial_{n}+(g f) \partial_{n} \partial_{n}-\partial_{n}(g f)=0 .
\end{gathered}
$$

Because chain complex $C_{n}^{N}(\bar{s})$ is exact at place $n$ and $-F_{n-1} \partial_{n}+I d-g f$ is in the kernel of this chain complex, it is also in the image, say, $\partial_{n+1} c_{n+1}=-F_{n-1} \partial_{n}+$ $I d-g f$. Then we declare $F_{n}(s)=c_{n+1}$. In fact here $c_{n+1}$ can be obtained by putting any, fixed, vertex of $s$ in front of $\left(-F_{n-1} \partial_{n}+I d-g f\right)$. Such constructed $F_{n}$ satisfies $\partial_{n+1} F_{n}+F_{n-1} \partial_{n}=I d-g f$. Our map is well defined as we constructed it on the basis of $C_{n}^{N}$. 


$$
\sum_{i=0}^{p-1}(-1)^{i} s_{p-1} d_{i} \sum_{i=p+2}^{n+1}(-1)^{i} s_{p} d_{i-1} \in C_{n-1}^{D},
$$

where the $\left(4^{\prime}\right)$ is the condition:

$$
\left(4^{\prime}\right) d_{p} s_{p}=d_{p+1} s_{p} \text { for any } p \leq n .
$$

If $\left(C_{n}, d_{i}, s_{i}\right)$ satisfies conditions $(1),(2),(3)$, and (4') it is called a weak simplicial module [Prz-5]. As condition (2) was not use in calculation it is useful also to consider $\left(C_{n}, d_{i}, s_{i}\right)$ satisfying the condition $(1),(3)$, and (4'), we call this a weak-pseudo-simplicial module.

We strengthen the above calculation by considering the sequence of modules $F_{n}^{i}=\operatorname{span}\left(s_{0}\left(C_{n-1}\right), s_{1}\left(C_{n-1}\right), \ldots, s_{i}\left(C_{n-1}\right)\right)$ and the filtration:

$$
0 \subset F_{n}^{0} \subset F_{n}^{1} \subset \ldots \subset F_{n}^{n-1}=C_{n}^{D} .
$$

Our calculation gives $\partial_{n}\left(s_{p}\left(C_{n-1}\right)\right) \subset \operatorname{span}\left(s_{p-1}\left(C_{n-1}\right), s_{p}\left(C_{n-1}\right)\right)$ and consequently:

Corollary 3.4. If $\left(C_{n}, d_{i}, s_{i}\right)$ is a weak-pseudo-simplicial module then $\partial_{n}$ is filtration preserving, that is $\partial_{n}\left(F_{n}^{p}\right) \subset F_{n}^{p-1}$.

We will prove now the Eilenberg-Mac Lane theorem that the degenerate complex $\left(C_{n}^{D}, \partial_{n}\right)$ is acyclic, watching on the way which axioms are used. For a filtration $\left(F_{n}^{p}\right)$ the associated graded module is defined to be $\left\{G r_{n}^{p}=F_{n}^{p} / F_{n}^{p-1}\right\}$. We prove first that the chain complex $\left\{G r_{n}^{p}\right\}$ is acyclic for any $p$.

Lemma 3.5. Let $\left(C_{n}, d_{i}, s_{i}\right)$ satisfies the conditions (1), (2"), (3), and (4) where

$$
\text { (2") } s_{p-1} s_{p-1}=s_{p} s_{p-1} \text { for every } 0<p \leq n \text {. }
$$

We call such $\left(C_{n}, d_{i}, s_{i}\right)$ a co-almost-simplicial module. Then the chain complex $\left\{G r_{n}^{p}=F_{n}^{p} / F_{n}^{p-1}\right\}$ is acyclic, in particular $H_{n}\left(\left\{G r_{n}^{p}\right\}\right)=0$, and homology of $F_{n}^{p}$ and $F_{n}^{p-1}$ are isomorphic.

Proof. The classical idea of Eilenberg and Mac Lane is to use the degenerate map $s_{p}$ as a chain homotopy (we follow [Lod-1]):

It suffices to show that $\left(\partial s_{p}+s_{p} \partial\right) s_{p}=(-1)^{p} s_{p}$ modulo $s_{p-1} C_{n-1}$, so the map $s_{p}$ is a chain homotopy between $(-1)^{p} I d$ and the zero map on $G r_{n}^{p}$. In the calculation we stress which axioms are used:

$$
\begin{gathered}
\left(\partial s_{p}+s_{p} \partial\right) s_{p}=\left(\sum_{i=0}^{n+1}(-1)^{i} d_{i} s_{p}+s_{p} \sum_{i=0}^{n}(-1)^{i} d_{i}\right) s_{p}= \\
\sum_{i=0}^{p-1}(-1)^{i} d_{i} s_{p} s_{p}+(-1)^{p}\left(d_{p} s_{p}-d_{p+1} s_{p}\right) s_{p}+\sum_{i=p+2}^{n+1}(-1)^{i} d_{i} s_{p} s_{p}+
\end{gathered}
$$




$$
\begin{aligned}
& \sum_{i=0}^{p-1}(-1)^{i} s_{p} d_{i} s_{p}+(-1)^{p} s_{p}\left(d_{p} s_{p}-d_{p+1} s_{p}\right)+\sum_{i=p+2}^{n}(-1)^{i} s_{p} d_{i} s_{p} \stackrel{(3)}{=} \\
& \sum_{i=0}^{p-1}(-1)^{i} s_{p-1} s_{p-1} d_{i}+(-1)^{p}\left(d_{p} s_{p}-d_{p+1} s_{p}\right) s_{p}+\sum_{i=p+2}^{n+1}(-1)^{i} s_{p} d_{i-1} s_{p}+ \\
& \sum_{i=0}^{p-1}(-1)^{i} s_{p} s_{p-1} d_{i}+(-1)^{p} s_{p}\left(d_{p} s_{p}-d_{p+1} s_{p}\right)+\sum_{i=p+2}^{n}(-1)^{i} s_{p} d_{i} s_{p}= \\
& \sum_{i=0}^{p-1}(-1)^{i} s_{p-1} s_{p-1} d_{i}+(-1)^{p}\left(d_{p} s_{p}-d_{p+1} s_{p}\right) s_{p}+(-1)^{p} s_{p} d_{p+1} s_{p}+ \\
& \sum_{i=0}^{p-1}(-1)^{i} s_{p} s_{p-1} d_{i}+(-1)^{p} s_{p}\left(d_{p} s_{p}-d_{p+1} s_{p}\right) \stackrel{d_{p} s_{p}=d_{p+1} s_{p}}{=} \\
& \sum_{i=0}^{p-1}(-1)^{i} s_{p-1} s_{p-1} d_{i}+\sum_{i=0}^{p-1}(-1)^{i} s_{p} s_{p-1} d_{i}+(-1)^{p} s_{p} d_{p+1} s_{p} \stackrel{(2)}{=} \\
& 2 \sum_{i=0}^{p-1}(-1)^{i} s_{p-1} s_{p-1} d_{i}+(-1)^{p} s_{p} d_{p+1} s_{p} \quad \stackrel{\bmod 2 s_{p-1} M_{n-1}}{=} \\
& (-1)^{p} s_{p} d_{p+1} s_{p} \stackrel{d_{p+1} s_{p}=I d}{=}(-1)^{p} s_{p}
\end{aligned}
$$

Now consider the short exact sequence $0 \rightarrow F^{p-1} \rightarrow F^{p} \rightarrow F^{p} / F^{p-1} \rightarrow$ 0 and the corresponding long exact sequence of homology:

$$
\ldots \rightarrow H_{n+1}\left(F^{p} / F^{p-1}\right) \rightarrow H_{n}\left(F^{p-1}\right) \rightarrow H_{n}\left(F^{p}\right) \rightarrow H_{n}\left(F^{p} / F^{p-1}\right) \rightarrow \ldots
$$

Thus because homology of $F^{p} / F^{p-1}$ is trivial we obtain isomorphism $H_{n}\left(F^{p-1}\right) \rightarrow H_{n}\left(F^{p}\right)$. In conclusion, by induction on $p$ we get the Eilenberg-Mac Lane result: $H_{n}^{D}(C)=0$.

From our proof follows that working modulo $2 s_{p-1} M_{n-1}$, e.g. modulo 2 , gives directly $H_{n}\left(F^{p}\right)=0$. Also from axiom (2) we took only $s_{p} s_{p-1}=s_{p-1} s_{p-1}$ that is axiom (2").

The above consideration do not work for a distributive case (the axiom (4) usually does not hold as explained in Section 6] (see [CPP, $\mathrm{Pr}-\mathrm{Pu}-1$, $\mathrm{P}-\mathrm{S}]$. We proved however that the degenerate part of quandle homology can be obtained from the normalized one via Künneth type formula, see [Pr-Pu-2]). 
3.3. Bicomplex for a degenerate subcomplex $C_{n}^{D}$. One more important observation follows from our calculations. If $\left(C_{n}, d_{i}, s_{i}\right)$ is a weak simplicial module 13 (i.e. conditions (1)-(3),(4') hold then the formula

$$
\partial_{n} s_{p}=\sum_{i=0}^{p-1}(-1)^{i} s_{p-1} d_{i}+\sum_{i=p+2}^{n}(-1)^{i} s_{p} d_{i-1}
$$

allows us to define a bicomplex with entries $E_{p, q}^{0}=G r_{n, p}=F_{n}^{p} / F_{n}^{p-1}$, $n=p+q$, with horizontal and vertical boundary operation: $\partial_{p, q}^{h}=$ $\sum_{i=0}^{p-1}(-1)^{i}$ and $\partial_{p, q}^{v}=\sum_{i=p+2}^{n}(-1)^{i} s_{p} d_{i-1}$ with $\partial_{p, q-1}^{h} \partial_{p, q}^{v}=-\partial_{p-1, q}^{v} \partial_{p, q}^{h}$ : $E_{p, q}^{0} \rightarrow E_{p-1, q-1}^{0}$; see Figure 3.1.

$$
\begin{aligned}
& \downarrow \partial^{v} \quad \downarrow \partial^{v} \quad \downarrow \partial^{v} \\
& \ldots \stackrel{d^{h}}{\leftarrow} \quad E_{p-1, q+1}^{0} \stackrel{\partial^{h}}{\leftarrow} \quad E_{p, q+1}^{0} \stackrel{\partial^{h}}{\leftarrow} \quad E_{p+1, q+1}^{0} \stackrel{\partial^{h}}{\leftarrow} \ldots \\
& \downarrow \partial^{v} \quad \downarrow \partial^{v} \quad \downarrow \partial^{v}
\end{aligned}
$$

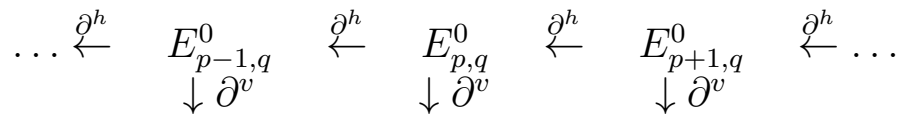

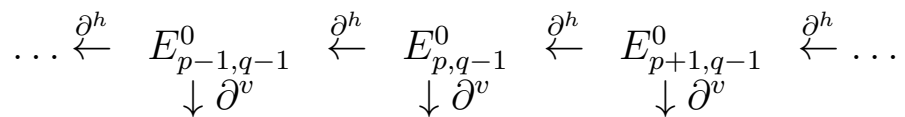

Figure 3.1; Bicomplex $\left(E_{p, q}^{0}, \partial^{v}, \partial^{h}\right)$

The bicomplex $\left(E_{p, q}^{0}, \partial^{v}, \partial^{h}\right)$ yields a spectral sequence, in fact two spectral sequences: starting from columns, that is ${ }^{c} E_{p q}^{1}=\frac{\operatorname{ker}\left(\partial^{v}: E_{p q}^{0} \rightarrow E_{p, q-1}^{0}\right)}{i m\left(\partial^{v}: E_{p, q+1}^{0} \rightarrow E_{p q}^{0}\right)}$, and the spectral sequence starting from rows: ${ }^{r} E_{p q}^{1}=\frac{\operatorname{ker}\left(\partial^{h}: E_{p q}^{0} \rightarrow E_{p-1, q}^{0}\right)}{i m\left(\partial^{h}: E_{p+1, q}^{0} \rightarrow E_{p q}^{0}\right)}$ which can be used to analyze homology of $\operatorname{Gr}\left(C^{D}\right)$ and $\left(C_{n}\right)$, see [Pr-Pu-2] for an application in the distributive case.

3.4. Homology with coefficients in a $k$-Mod functor. Each individual abstract simplicial complex $K=(V, \mathcal{P})$ is a small category 14 with simplexes as objects and inclusions of simplexes, $s \subset s^{\prime}$, as morphisms. As usually $K^{o p}$ will denote the opposite category so restrictions, $s \supset s^{\prime}$, are morphisms, more precisely $\operatorname{Mor}_{K^{o p}}\left(s, s^{\prime}\right)$ is empty if $s$ does not contain $s^{\prime}$ and otherwise $\operatorname{Mor}_{K^{o p}}\left(s, s^{\prime}\right)$ has one morphism denoted by $\left(s \supset s^{\prime}\right)$. Now for any (covariant) functor $\mathcal{F}: K^{o p} \rightarrow k-\mathrm{Mod}$, where $k$-Mod is a category of modules over a commutative ring $k$, we can define oriented homology $H_{n}^{\text {ori }}(K, \mathcal{F})$ of an abstract simplicial complex $K$ with coefficients in $\mathcal{F}$, as follows:

\footnotetext{
${ }^{13}$ In fact a pseudo weak simplicial module suffices, i.e. conditions $(1),(3),\left(4^{\prime}\right)$.

${ }^{14}$ Category is called small if objects form a set.
} 
Definition 3.6. Let $K=(V, P)$ be an abstract simplicial complex with ordered vertice 15 and $\mathcal{F}: K^{o p} \rightarrow k-M o d$ a functor. We define the presimplicial module $\left(C_{n}^{\text {ori }}(K, \mathcal{F}), d_{i}\right)$ as follows:

$$
C_{n}^{\text {ori }}(K, \mathcal{F})=\bigoplus_{\operatorname{dim}(s)=n} \mathcal{F}(s)
$$

the face map $d_{i}: C_{n}^{\text {ori }}(K, \mathcal{F}) \rightarrow C_{n-1}^{\text {ori }}(K, \mathcal{F})$ is defined by

$$
d_{i}=\mathcal{F}\left(s \supset\left(s-x_{i}\right)\right) \text { where } s=\left(x_{0}, \ldots, x_{n}\right) \text { and } x_{i}<x_{i+1}
$$

as usually for presimplicial modules $\partial_{n}=\sum_{i=1}^{n}(-1)^{i} d_{i}$ and $\left(C_{n}^{\text {ori }}(K, \mathcal{F}), \partial_{i}\right)$ is a chain complex whose homology is denoted by $H_{n}^{\text {ori }}(K, \mathcal{F})$.

The above definition can be thought of as a twisted version of an oriented homology of abstract simplicial complexes. Similarly we can define ordered homology of $(K, \mathcal{F})$ but oriented and ordered homology with coefficient in a functor are not necessarily isomorphic.

Definition 3.6 is related to more general Definition 3.7 on homology of a small category with a functor coefficient, usually thought to be first given by Wat, who in turn refers to the earlier paper Dehe in the case of the category of posets.

Definition 3.7. Let $\mathcal{P}$ be as small category (i.e. objects, $P=O b(\mathcal{P})$ form a set), and let $\mathcal{F}: \mathcal{P} \rightarrow k$-Mod be a functor from $\mathcal{P}$ to the category of modules over a commutative ring $k$. We call the sequence of objects and functors, $x_{0} \stackrel{f_{0}}{\rightarrow} x_{1} \stackrel{f_{1}}{\rightarrow} \ldots \stackrel{f_{n-1}}{\rightarrow} x_{n}$ an $n$-chain (more formally $n$-chain in the nerve of the category). We define the chain complex $C_{*}(\mathcal{P}, \mathcal{F})$ as follows:

$$
C_{n}=\bigoplus_{x_{0} \stackrel{f_{0}}{\rightarrow} x_{1} \stackrel{f_{1}}{\rightarrow} \stackrel{f_{n-1} x_{n}}{\rightarrow}} \mathcal{F}\left(x_{0}\right)
$$

where the sum is taken over all n-chains.

The boundary operation $\partial_{n}: C_{n}(\mathcal{P}, \mathcal{F}) \rightarrow C_{n+1}(\mathcal{P}, \mathcal{F})$ is an alternative sum of face maps, $\partial_{n}=\sum_{i=0}^{n}(-1)^{i} d_{i}$, where $d_{i}$ are given by:

$$
\begin{aligned}
& d_{0}\left(\lambda ; x_{0}\right.\left.\stackrel{f_{0}}{\rightarrow} x_{1} \stackrel{f_{1}}{\rightarrow} \ldots \stackrel{f_{n-1}}{\rightarrow} x_{n}\right)=\left(\mathcal{F}\left(x_{0} \stackrel{f_{0}}{\rightarrow} x_{1}\right)(\lambda) ; x_{1} \stackrel{f_{1}}{\rightarrow} \ldots \stackrel{f_{n-1}}{\rightarrow} x_{n}\right), \\
& d_{i}\left(\lambda ; x_{0} \stackrel{f_{0}}{\rightarrow} x_{1} \stackrel{f_{1}}{\rightarrow} \ldots \stackrel{f_{n-1}}{\rightarrow} x_{n}\right)=\left(\lambda ; x_{0} \stackrel{f_{0}}{\rightarrow} x_{1} \stackrel{f_{1}}{\rightarrow} \ldots \rightarrow x_{i-1} \stackrel{f_{i} f_{i-1}}{\rightarrow} x_{i+1} \rightarrow \ldots \stackrel{f_{n-1}}{\rightarrow} x_{n}\right)
\end{aligned}
$$

\footnotetext{
${ }^{15}$ It suffices to have $V$ partially ordered as long as for any simplex $s=\left(x_{0}, \ldots, x_{n}\right)$ the partial order on $V$ restricts to linear order on vertices of $s$. Even better we do not need a partial order, it suffices that vertices of every simplex are ordered in such a way that if $s_{1} \subset s_{2}$ then the ordering of vertices of $s_{1}$ is a restriction of the ordering of vertices of $s_{2}$.
} 
for $0<i<n$, and

$$
d_{n}\left(\lambda ; x_{0} \stackrel{f_{0}}{\rightarrow} x_{1} \stackrel{f_{1}}{\rightarrow} \ldots \stackrel{f_{n-1}}{\rightarrow} x_{n}\right)=\left(\lambda ; x_{0} \stackrel{f_{0}}{\rightarrow} x_{1} \stackrel{f_{1}}{\rightarrow} \ldots \stackrel{f_{n-2}}{\rightarrow} x_{n-1}\right) .
$$

We denote by $H_{n}(\mathcal{P}, \mathcal{F})$ the homology yielded by the above chain complex, and call this the homology of a small category $\mathcal{P}$ with coefficients in a functor $\mathcal{F}$.

Similarly, if $\mathcal{F}^{\prime}: \mathcal{P} \rightarrow k-$ Mod is a contravariant functor we may define a homology $H_{n}\left(\mathcal{P} ; \mathcal{F}^{\prime}\right)$, starting from

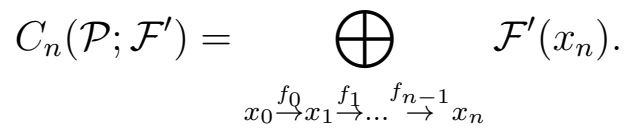

In particular, $d_{n}\left(x_{0} \stackrel{f_{0}}{\rightarrow} x_{1} \stackrel{f_{1}}{\rightarrow} \ldots \stackrel{f_{n-1}}{\rightarrow} x_{n} ; \lambda\right)=$ $\left.\left(x_{0} \stackrel{f_{0}}{\rightarrow} x_{1} \stackrel{f_{1}}{\rightarrow} \ldots \stackrel{f_{n-2}}{\rightarrow} x_{n-1}\right) ; \mathcal{F}^{\prime}\left(x_{n-1} \stackrel{f_{n-1}}{\rightarrow} x_{n}\right)(\lambda)\right)$, where $\lambda \in \mathcal{F}^{\prime}\left(x_{n}\right)$. One can also consider both functors, $\mathcal{F}$ and $\mathcal{F}^{\prime}$ in the definition starting from

$$
C_{n}\left(\mathcal{P} ; \mathcal{F}, \mathcal{F}^{\prime}\right)=\bigoplus_{x_{0} \stackrel{f_{0}}{\rightarrow} x_{1} \rightarrow \ldots \stackrel{f_{1}}{\rightarrow}{ }^{f_{n-1}} x_{n}} \mathcal{F}^{\prime}\left(x_{n}\right) \otimes \mathcal{F}\left(x_{0}\right) ;
$$

compare Definition 4.6. We can also start from from a bifunctor $\mathcal{D}$ : $\mathcal{P}^{o p} \times \mathcal{P} \rightarrow k-M o d$ and mimic the definition of the Hochschild homology (Section 5) [Lod-1].

Remark 3.8. Any subcategory $\mathcal{P}^{\prime}$ of $\mathcal{P}$ has its chain complex, and homology (we use the functor $\mathcal{F}^{\prime}=\mathcal{F} / \mathcal{P}^{\prime}$, that is, the restriction of $\mathcal{F}$ to $\left.\mathcal{P}^{\prime}\right) . \quad C_{*}\left(\mathcal{P}^{\prime}, \mathcal{F}^{\prime}\right)$ is a subchain complex of $C_{*}(\mathcal{P}, \mathcal{F})$ so we can consider the short exact sequence of chain complexes:

$$
0 \rightarrow C_{n}(\mathcal{P}, \mathcal{F}) \rightarrow C_{n}\left(\mathcal{P}^{\prime}, \mathcal{F}^{\prime}\right) \rightarrow C_{n}(\mathcal{P}, \mathcal{F}) / C_{n}\left(\mathcal{P}^{\prime}, \mathcal{F}^{\prime}\right) \rightarrow 0
$$

and yielded by it the long exact sequence of homology.

The pair $\left(C_{n}, d_{i}\right)$ form a presimplicial module by associativity of morphisms of a category and properties of a functor. More generally we have:

Proposition 3.9. Let $s_{i}: C_{n} \rightarrow C_{n+1}$ be a map inserting identity morphism on the ith place in the nth chain of of the nerve of the category, that is

$$
s_{i}\left(\left(\lambda ; x_{0} \stackrel{f_{0}}{\rightarrow} \ldots \stackrel{f_{n-1}}{\rightarrow} x_{n}\right)=\left(\lambda ; x_{0} \stackrel{f_{0}}{\rightarrow} x_{i} \stackrel{I d_{x_{i}}}{\rightarrow} x_{i} \ldots \stackrel{f_{n-1}}{\rightarrow} x_{n}\right) .\right.
$$

Then $\left(C_{n}, d_{i}, s_{i}\right)$ is a simplicial module.

The classical example is the homology of a simplicial complex with constant coefficients, that is $\mathcal{F}(s)=k$ and $\mathcal{F}(f)=I d_{k}$; in that case we write $H_{n}(K, \mathcal{F})=H_{n}(K, k)$ or just $H_{n}(K)$ if $k=\mathbb{Z}$. Related to this 
example is homology of posets: if $\mathcal{P}$ is a small category and for any objects $x$ and $y, \operatorname{Mor}(x, y)$ has at most one element and additionally if $\operatorname{Mor}(x, y) \neq \emptyset$ and $\operatorname{Mor}(y, x) \neq \emptyset$ then $x=y$, then $\mathcal{P}$ is a poset with $x \leq y$ iff $\operatorname{Mor}(x, y) \neq \emptyset$.

Another classical example concerns homology of groups, where the category has one object and $G$ morphisms (interpreted as multiplication by elements of $G$ [Bro, that is the morphism $g: G \rightarrow G$ is given by $g(h)=h g$ ); compare Section 4 .

More recent example is motivated by Khovanov homology so we call a related functor $\mathcal{F}_{(D, A, M)}$, a Khovanov functor. The functor depends on a choice of an $k$-Frobenius algebra $A, A$-Frobenius bimodule $M$ and a link diagram (possibly virtual link, or a link diagram on a surface, $L$ (equivalently we can work with graphs on a surface). Here for simplicity we assume that $M=A$ is an abelian Frobenius $k$-algebra $\sqrt{16}$ and $D$ is a classical link diagram.

Definition 3.10. For a link diagram $D$, let $V$ be the set of its crossings (in some order), and $P=2^{V}$. Thus $K=(V, P)$ is a simplex (we allow also the empty, -1-dimensional simplex). Let $A$ be a Frobenius algebra with a multiplication $\mu$ and a co-multiplication $\Delta$ (e.g. $A=Z[x] /\left(x^{m}\right)$, $\left.\Delta(1)=\sum_{i+j=m-1} x^{i} \otimes x^{j}\right)$. We define a functor $\mathcal{F}_{D, A}: K \rightarrow k$-Mod as follows. For any $s \in P$ we identify $s$ with a Kauffman state, where $s(v)=1$ (i.e. $\searrow$ ) iff $v \in s$. We denote by $D_{s}$ the collection of circles obtained from $D$ by smoothing it according to $s$, and by $\left|D_{s}\right|$ the number of circles in $D_{s}$. Then we define $\mathcal{F}(s)=A^{\otimes\left|D_{s}\right|}$. To define $\mathcal{F}\left(s \supset\left(s-v_{i}\right)\right)$ we first decorate circles of $D_{s}$ by algebra $A$, (that is each circle by one copy of $A)$; then we have two cases:

( $\mu$ ) $\left|D_{s-v_{i}}\right|=\left|D_{s}\right|-1$, thus two circles are glued together when we switch the state at $v_{i}$. In this case we multiply the element associated with glued circles (we use the fact that $A$ is commutative).

$(\Delta)\left|D_{s-v_{i}}\right|=\left|D_{s}\right|+1$, thus a circle of $D_{s}$ is split so we apply to the element of $A$ associated to this circle a co-multiplication (we use the fact that $\Delta$ is co-commutative). $\mathcal{F}$ is a functor as $A$ is a commutative Frobenius algebra.

This approach to Khovanov homology was first sketched in [Prz-4], where Khovanov homology was connected to Hochschild homology.

\footnotetext{
${ }^{16} A$ is a $k$ module with associative and commutative multiplication, $\mu$, with co-associative and co-commutative co-multiplication, $\Delta$, satisfying the Frobenius condition, that is $\Delta \mu=(\mu \times I d)(I d \times \Delta)$; graphically: $Y=N$. There is no need for unit and counit.
} 
It is a classical result that homology of a baricentric subdivision of an abstract simplicial complex is isomorphic to the homology of the complex. This was an ingredient of original proofs of topological invariance of homology. The generalization of the result holds also for a homology of a simplicial complex $\mathcal{K}$ with a coefficient in a functor and the homology of $\mathcal{K}$ treated as a small category. I was informed by S. Betley and Jolanta Słomińska about at least three proof of the fact, compare [Slo]. We are writing, with my student Jing Wang detailed survey with the proof following closely the classical proof with constant coefficients (in essence it is another case of acyclic model theorem of Eilenberg and Zilber [E-Z]).

In the next few sections we discuss homology related to various magmas (e.g. associative and right distributive) and look for the common traits, for example presimplicial or simplicial module structure, geometric realization etc.

\section{Group homology of a Semigroup}

In the homology of abstract simplicial complexes, the set of vertices, $X$, has no algebraic structure or, as we will see later, we can treat $X$ as a magma with the trivial operation $*_{0}, x *_{0} y=x$. We will now discuss homology of magma $(X, *)$ equipped with some specific structure, e.g. associativity, Jacobi identity, or distributivity.

According to [Bro]: The cohomology theory of groups arose from both topological and algebraic sources. The starting point for the topological aspect of the theory was the work of Hurewicz ([Hur], 1936 on "aspherical spaces". About a year earlier, Hurewicz had introduced the higher homotopy groups $\pi_{n} X$ of a space $X(n \geq 2)$. He now singled out for study those path-connected spaces $X$ whose higher homotopy groups are all trivial, but whose fundamental group $\pi=\pi_{1} X$ need not be trivial. Such spaces are called aspherical. Hurewicz proved, among other things, that the homotopy type of an aspherical pace $X$ is completely determined by its fundamental group $\pi$. ... Hopf ([Hop, 1942)... expressed $\mathrm{H}_{2} \pi$ in purely algebraic terms...

Let $(X, *)$ be a semigroup that is a set with associative binary operation. We associate with $(X, *)$ a presimplicial set, presimplicial module, chain complex, group homology and geometric realization as follows:

Definition 4.1. (i) Let $X_{n}=X^{n}$ and $d_{i}: X_{n} \rightarrow X_{n-1}$ for $0 \leq i \leq$ $n$ is given by:

$$
\begin{gathered}
d_{0}\left(x_{1}, x_{2}, \ldots, x_{n}\right)=\left(x_{2}, \ldots, x_{n}\right), \\
d_{i}\left(x_{1}, \ldots, x_{n}\right)=\left(x_{1}, \ldots, x_{i-1}, x_{i} * x_{i+1}, x_{i+2}, \ldots, x_{n}\right) \text { for } 0<i<n,
\end{gathered}
$$




$$
d_{0}\left(x_{1}, \ldots, x_{n-1}, x_{n}\right)=\left(x_{1},, \ldots, x_{n-1}\right) .
$$

Then $\left(X_{n}, d_{i}\right)$ is a presimplicial set.

(ii) If we choose a commutative ring $k$ and consider $C_{n}=k X^{n}$ and $d_{i}: C_{n} \rightarrow C_{n-1}$ the unique extension of the map $d_{i}$ from (i) then $\left(C_{n}, d_{i}\right)$ is a presimplicial module.

(iii) If $\partial_{n}=\sum_{i=0}^{n}(-1)^{i} d_{i}$, then $\left(C_{n}, \partial_{n}\right)$ is a chain complex; its homology are called group homology of a semigroup $X$ and denoted by $H_{n}(X ; k)$ or just $H_{n}(X)$ if $k=\mathbb{Z}$.

(iv) A presimplicial set has a standard geometric realization, $B X$ (as a CW-complex 17). Thus the semigroup homology has a natural interpretation as a homology of a $C W$-complex [Bro].

Definition 4.1 has a classical generalization, when a semigroup $(X ; *)$ is augmented by an $X$-right-semigroup-set $E$, that is a set with the right action (also denoted by $*$ ) of $X$ on $E$ such that $(e * a) * b=e *(a * b)$.

Definition 4.2. (i) Let $X_{n}=E \times X^{n}$ and $d_{i}: X_{n} \rightarrow X_{n-1}$ for $0 \leq i \leq n$ is given by:

$$
\begin{gathered}
d_{0}\left(e, x_{1}, x_{2}, \ldots, x_{n}\right)=\left(e * x_{1}, x_{2}, \ldots, x_{n}\right), \\
d_{i}\left(e, x_{1}, \ldots, x_{n}\right)=\left(e, x_{1}, \ldots, x_{i-1}, x_{i} * x_{i+1}, x_{i+2}, \ldots, x_{n}\right) \text { for } 0<i<n, \\
d_{n}\left(e, x_{1}, \ldots, x_{n-1}, x_{n}\right)=\left(e, x_{1}, \ldots, x_{n-1}\right) .
\end{gathered}
$$

Then $\left(X_{n}, d_{i}\right)$ is a presimplicial set.

(ii) For $C_{n}=k\left(E \times X^{n}\right),\left(C_{n}, d_{i}\right)$ is a presimplicial module and $\left(C_{n}, \partial_{n}\right)$ is a chain complex with homology denoted by $H_{n}(X, E)$ and geometric realization $B(X, E)$.

If $E$ has one element then we get the case of Definition 4.1.

Definition 4.2 has further generalization if, in addition to a semigroup $(X ; *)$ we have the right $X$-set $E_{0}$ and the left $X$ set $E_{w}$ (here we need $(a *(b * e)=(a * b) * e)$, compare [Ca-E], Chapter X.

Definition 4.3. (i) Let $X_{n}=E_{0} \times X^{n} \times E_{w}$ and $d_{i}: X_{n} \rightarrow X_{n-1}$ for $0 \leq i \leq n$ is given by:

$$
\begin{aligned}
& \left.\quad d_{0}\left(e_{0}, x_{1}, x_{2}, \ldots, x_{n}, e_{n+1}\right)=\left(e * x_{0}, x_{2}, \ldots, x_{n}, e_{n+1}\right)\right), \\
& d_{i}\left(e_{0}, x_{1}, \ldots, x_{n}, e_{n+1}\right)=\left(e_{0}, x_{1}, \ldots, x_{i-1}, x_{i} * x_{i+1}, x_{i+2}, \ldots, x_{n}, e_{n+1}\right) \text { for } 0<i<n, \\
& d_{n}\left(e_{0}, x_{1}, \ldots, x_{n-1}, x_{n}, e_{n+1}\right)=\left(e_{0}, x_{1}, \ldots, x_{n-1}, x_{n} * e_{n+1}\right) . \\
& \text { Then }\left(X_{n}, d_{i}\right) \text { is a presimplicial set. We call this pre-simplicial } \\
& \text { set a "two walls" presimplicial set due to visualization in Figure } \\
& \text { 4.1. }
\end{aligned}
$$

\footnotetext{
${ }^{17} B X$ can be made into geometric simplicial complex by second baricentric subdivision because $B X$ by the construction is glued from simplexes (such a space is called a $\Delta$-complex in Hat]), see Section [13, e.g. Definition 13.1
} 
(ii) For $C_{n}=k\left(E_{0} \times X^{n} \times E_{w}\right),\left(C_{n}, d_{i}\right)$ is a presimplicial module and $\left(C_{n}, \partial_{n}\right)$ is a chain complex with homology denoted by $H_{n}\left(X, E_{0}, E_{w}\right)$, and geometric realization $B\left(X, E_{0}, E_{w}\right)$.

If $E_{w}$ has one element then we get the case of Definition 4.2

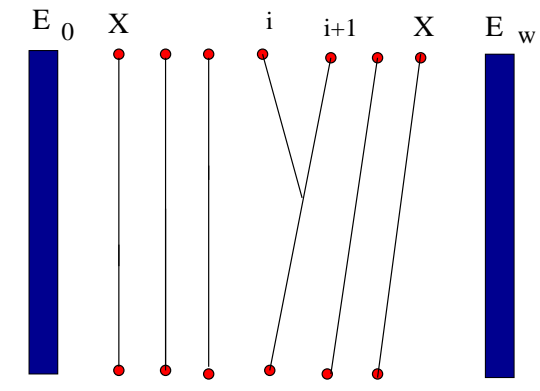

Figure 4.1; i'th face map in "two walls" presimplicial set for a semigroup

A version of Definition 4.3 when we assume that $E_{0}=E_{w}$ and $\left(e_{1} *\right.$ $x) * e_{2}=e_{1} *\left(x * e_{2}\right)$ that is $E_{0}$ is an $X$-biset leads to the Hochschild homology. In particular, for a semigroup $(X ; *)$ we have:

Definition 4.4. Let $(X ; *)$ be a semigroup and $E$ an $X$-biset then:

(i) Let $X_{n}=E \times X^{n}$ and $d_{i}: X_{n} \rightarrow X_{n-1}$ for $0 \leq i \leq n$ is given by:

$$
\begin{gathered}
d_{0}\left(e, x_{1}, x_{2}, \ldots, x_{n}\right)=\left(e * x_{1}, x_{2}, \ldots, x_{n}\right), \\
d_{i}\left(e, x_{1}, \ldots, x_{n}\right)=\left(e, x_{1}, \ldots, x_{i-1}, x_{i} * x_{i+1}, x_{i+2}, \ldots, x_{n}\right) \text { for } 0<i<n, \\
d_{n}\left(e, x_{1}, \ldots, x_{n-1}, x_{n}\right)=\left(x_{n} * e, x_{1}, \ldots, x_{n-1}\right) .
\end{gathered}
$$

Then $\left(X_{n}, d_{i}\right)$ is a presimplicial set.

(ii) For $C_{n}=k\left(E \times X^{n}\right),\left(C_{n}, d_{i}\right)$ is a presimplicial module and $\left(C_{n}, \partial_{n}\right)$ is a chain complex with (Hochschild) homology denoted by $H_{n}(X, E)$ and geometric realization $B H(X, E)$.

(iii) If $E_{0}$ is an $X$-right-semigroup-set and $E_{w}$ is an $X$-right-semigroupset then we can take $E=E_{w} \times E_{0}$ and $E$ has a natural structure of an X-biset. Thus the concepts of a "two-wall" semigroup homology and Hochschild semigroup homology are equivalent.

If $(X ; *)$ is a monoid (with a unit element 1 ) then we say that the set $E$ is $X$-right-monoid-set if it is $X$-right-monoid-set and additionally $e * 1=e$ for any $e \in E$ (that is 1 acts trivially on $E$ from the right. Similarly we define $X$-left-monoid-set (e.g. $1 * e=e$ ). For a monoid the presimplicial sets (modules) described in Definitions 4.2, 4.3, and 4.4 are in fact simplicial sets (modules) with the degeneracy maps $s_{i}$ placing 1 between $x_{i}$ and $x_{i+1}$, for example in the case of 4.3 .

$$
s_{0}\left(e_{0}, x_{1}, x_{2}, \ldots, x_{n}, e_{n+1}\right)=\left(e_{0}, 1, e_{0}, x_{1}, x_{2}, \ldots, x_{n}, e_{n+1}\right),
$$




$$
\begin{gathered}
s_{i}\left(e_{0}, x_{1}, x_{2}, \ldots, x_{n}, e_{n+1}\right)=\left(e_{0}, x_{1}, \ldots, x_{i}, 1, x_{i+1}, \ldots, x_{n}, e_{n+1}\right) \text { for } 0<i<n, \\
s_{n}\left(e_{0}, x_{1}, x_{2}, \ldots, x_{n}, e_{n+1}\right)=\left(e_{0}, x_{1}, x_{2}, \ldots, x_{n}, 1, e_{n+1}\right) .
\end{gathered}
$$

Example 4.5. We can check that $d_{i} d_{i+1}=d_{i} d_{i}(0<i<n)$ if and only if $*$ is associative.

Furthermore, $d_{0} d_{1}=d_{0} d_{0}$ iff $\left(e_{0} * x_{1}\right) * x_{2}=e_{0} *\left(x_{1} * x_{2}\right)$ that is $E_{0}$ is an $X$-right-semigroup-set.

Similarly $d_{n-1} d_{n}=d_{n-1} d_{n-1}$ iff $E_{w}$ is an $X$-left-semigroup-set.

Let $\partial^{(\ell)}$ be a boundary map obtained from the group homology boundary operation by dropping the first term from the sum. Analogously, let $\partial^{(r)}$ be a boundary map obtained from the group homology boundary operation by dropping the last term from the sum. It is a classical observation that $\left(C_{n}, \partial^{(\ell}\right)$ and $\left(C_{n}, \partial^{(r)}\right)$ are acyclic for a group (or a monoid). We show this in a slightly more general context of weak simplicial modules (used later in the distributive case) in Section 6. It would be of interest to analyze homology of $\left(C_{n}, \partial^{(\ell}\right)$ for a semigroup without identity. Can it have a torsion?.

Our definition (in the presented form) can be generalized to any $k$ algebra $V$ not only $V=k X$ Below we give the definition for "two wall" $k$-algebra, and in the next section we describe the mainstream Hochschild homology of $k$-algebra closely related to group homology.

Definition 4.6. Let $A$ be a k-algebra which acts from the right on a $k$-module $M_{0}((m * x) * y=m *(x * y))$ and from the left on a $k$-module $M_{w}(x *(y * m)=(x * y) * m)$, that is $M_{0}$ is a right $A$-module and $M_{w}$ a left $A$-module. We define chain groups $C_{n}=M_{0} \otimes A^{\otimes n} \otimes M_{w}$ and face maps $d_{i}\left(x_{0}, x_{1}, \ldots, x_{n}, x_{n+1}\right)=\left(x_{0}, \ldots, x_{i} * x_{i+1}, \ldots, x_{n+1}\right), 0 \leq$ $i \leq n, x_{0} \in M_{0}, x_{n+1} \in M_{w}$, and $x_{i} \in A$ for $0<i \leq n$. Then $\left(C_{n}, d_{i}\right)$ is a presimplicial module and $\left(C_{n}, \partial_{n}\right)$, with $\partial_{n}=\sum_{i=0}^{n}(-1)^{i} d_{i}$ is a chain complex, whose homology is denoted by $H_{n}\left(A, M_{0}, M_{w}\right)$. If $A$ is a unitary algebra (with unit 1 ) then we define degenerate maps $s_{i}\left(x_{0}, x_{1}, \ldots, x_{n}, x_{n+1}\right)=\left(x_{0}, x_{1}, \ldots, x_{i}, 1, x_{i+1}, \ldots, x_{n}, x_{n+1}\right), 0 \leq i \leq n$, and one checks directly that $\left(C_{n}, d_{i}, s_{i}\right)$ is a simplicial module.

If we glue together $M_{0}$ and $M_{w}$ to get 2-sided module (A-bimodule) $M=M_{w} \otimes M_{0}$ we obtain Hochschild homology $H_{n}(A, M)$, Hoch, Lod-1] see Section 5 .

\section{Hochschild homology of A SEMigroup AND AN ALGEBRA}

Hochschild homology was created to have a homology theory of algebras, as before homology was defined only for (semi)groups, $G$, and (semi)group algebras $k G$ (Definition 4.6 is only afterthought with Hochschild homology in mind). The history of discovering homology for 
algebra is described in Mac Lane autobiography [Mac]: Given his topological background and enthusiasm, Eilenberg was perhaps the first person to see this clearly. He was in active touch with Gerhard Hochschild, who was then a student of Chevalley at Princeton. Eilenberg suggested that there ought to be a cohomology (and a homology) for algebras. This turned out to be the case, and the complex used to describe the cohomology of groups (i.e. the bar resolution) was adapted to define the Hochschild cohomology of algebras.

Nevertheless, we start from Hochschild homology of semigroups as it leads to a presimplicial set, while the general Hochschild homology gives a presimplicial module.

Let $(X ; *)$ be a semigroup and $E$ a two sided $X$-semigroup-set that is $(e * a) * b=e *(a * b),(a * e) * b=a *(e * b)$, and $(a * b) * e=a *(b * e)$ We define a Hochschild presimplicial module $\left\{C_{n}(X, E), d_{i}\right\}$ as follows [Hoch, Lod-1]: $C_{n}(X)=k\left(E \times X^{n}\right)$ and the Hochschild face map is given by $d_{i}: k\left(E \times X^{n}\right) \rightarrow k\left(E \times X^{n-1}\right.$ where $d_{0}\left(e_{0}, x_{1}, \ldots x_{n}\right)=$ $\left(e_{0} * x_{1}, x_{2}, \ldots, x_{n}\right)$, $d_{i}\left(e_{0}, x_{1}, \ldots x_{n}\right)=\left(e_{0}, x_{1}, \ldots, x_{i-1}, x_{i} * x_{i+1}, \ldots, x_{n}\right.$ for $0<i<n$, and $d_{n}\left(e_{0}, x_{1}, \ldots x_{n}\right)=\left(x_{n} * e_{0}, x_{1}, \ldots, x_{n-1}\right)$.

$\partial_{n}: \mathbb{Z} X^{n} \rightarrow \mathbb{Z} X^{n-1}$ is defined by:

$$
\begin{gathered}
\partial\left(x_{0}, x_{1}, \ldots x_{n}\right)= \\
\sum_{i=0}^{n-1}(-1)^{i}\left(x_{0}, \ldots, x_{i-1}, x_{i} * x_{i+1}, x_{i+2}, \ldots, x_{n}\right)+ \\
(-1)^{n}\left(x_{n} * x_{0}, x_{1}, \ldots x_{n-1}\right)
\end{gathered}
$$

The resulting homology is called the Hochschild homology of a semigroup $(X, *)$ and denoted by $H H_{n}(X)$ (introduced by Hochschild in $1945[$ Hoch $]$. It is useful to define $C_{-1}=\mathbb{Z}$ and define $\partial_{0}(x)=1$ to obtain the augmented Hochschild chain complex and augmented Hochschild homology.

Again if $(X, *)$ is a monoid then dropping the last term gives an acyclic chain complex.

Notice that $\partial_{n}=\sum_{i=0}^{n}(-1)^{i} d_{i}$, where $d_{i}\left(x_{0}, \ldots, x_{n}\right)=\left(x_{0}, \ldots, x_{i-1}, x_{i}\right.$ * $\left.x_{i+1}, x_{i+2}, \ldots, x_{n}\right)$, for $0 \leq i<n$ and $d_{n}\left(x_{0}, \ldots, x_{n}\right)=\left(x_{n} * x_{0}, \ldots, x_{n-1}\right)$.

Again, $\left(C_{n}, d_{i}\right)$ is a presimplicial module. If $(X, *)$ is a monoid, one can define $n+1$ homomorphisms $s_{i}: C_{n} \rightarrow C_{n+1}$, called degeneracy maps, by $s_{i}\left(x_{0}, \ldots, x_{n}\right)=\left(x_{0}, \ldots, x_{i}, 1, x_{i+1}, \ldots, x_{n}\right)$ (similarly, in the case of group homology of a semigroup, we put, $s_{i}\left(x_{1}, \ldots, x_{n}\right)=$ $\left.\left(x_{1}, \ldots, x_{i}, 1, x_{i+1}, \ldots, x_{n}\right)\right)$. We check that in both cases the following 
conditions hold:

$$
\begin{gathered}
\text { (1) } d_{i} d_{j}=d_{j-1} d_{i} \text { for } i<j . \\
\text { (2) } s_{i} s_{j}=s_{j+1} s_{i}, \quad 0 \leq i \leq j \leq n, \\
\text { (3) } d_{i} s_{j}= \begin{cases}s_{j-1} d_{i} & \text { if } i<j \\
s_{j} d_{i-1} & \text { if } i>j+1\end{cases} \\
\text { (4) } d_{i} s_{i}=d_{i+1} s_{i}=I d_{C_{n}} .
\end{gathered}
$$

$\left(C_{n}, d_{i}, s_{i}\right)$ satisfying conditions (1)-(4) above is called a simplicial module 18 (e.g. $Z$-module/abelian group). If we replace (4) by a weaker condition $d_{i} s_{i}=d_{i+1} s_{i}$ we deal with a weak simplicial module, the concept useful in the theory of homology of distributive structures (spindles or quandles).

As we already mentioned before Hochschild homology (and presimplicial module) can be defined for any algebra $A$ and two-sided $A$ module $M$. We put $C_{n}(A ; M)=M \otimes A^{\otimes n}$ and $d_{i}\left(m, x_{1}, \ldots, x_{n}\right)$ is given by:

$$
\begin{gathered}
d_{0}\left(m, x_{1}, \ldots, x_{n}\right)=\left(m x_{1}, x_{2}, \ldots, x_{n}\right) . \\
d_{i}\left(m, x_{1}, \ldots, x_{n}\right)=\left(m, x_{1}, \ldots, x_{i-1}, x_{i} x_{i+1}, x_{i+2}, \ldots, x_{n}\right) \text { for } 0<i<n, \text { and } \\
d_{n}\left(m, x_{1}, \ldots, x_{n}\right)=\left(x_{n} m, x_{1}, \ldots, x_{n-1}\right) .
\end{gathered}
$$

From associativity of $A$ and our action of $A$ on $M$ follows that $\left(C_{n}, d_{i}\right)$ is a presimplicial module. Furthermore, if $A$ is unitary we can define a simplicial module structure $\left(C_{n}, d_{i}, s_{i}\right)$, by putting $s_{i}\left(m, x_{1}, \ldots, x_{n}\right)=$ $\left(m, x_{1}, \ldots, x_{i-1}, 1, x_{i}, \ldots, x_{n}\right)$.

\section{Homology of Distributive Structures}

Recall that a shelf (or right distributive system $(\operatorname{RDS}))(X ; *)$ is a set $X$ with a right self-distributive binary operation $*: X \times X \rightarrow X$ (i.e. $(a * b) * c=(a * c) *(b * c))$.

We work, for simplicity, with chain complexes and homology over $\mathbb{Z}$ but we can replace $\mathbb{Z}$ by any commutative ring $k$ in our considerations.

We start from atomic definition, one term distributive homology, introduced in 2010 just before Knots in Poland III conference [Prz-5].

\footnotetext{
${ }^{18}$ The concept of a simplicial set was introduced by Eilenberg and Zilber who called it complete semi-simplicial complex; their semi-simplicial complex is now usually called presimplicial set [E-Z, May].
} 


\subsection{One-term distributive homology.}

Definition 6.1. We define a (one-term) distributive chain complex $\mathcal{C}^{(*)}$ as follows: $C_{n}=\mathbb{Z} X^{n+1}$ and the boundary operation $\partial_{n}^{(*)}: C_{n} \rightarrow C_{n-1}$ is given by:

$$
\begin{gathered}
\partial_{n}^{(*)}\left(x_{0}, \ldots, x_{n}\right)=\left(x_{1}, \ldots, x_{n}\right)+ \\
\sum_{i=1}^{n}(-1)^{i}\left(x_{0} * x_{i}, \ldots, x_{i-1} * x_{i}, x_{i+1}, \ldots, x_{n}\right) .
\end{gathered}
$$

The homology of this chain complex is called a one-term distributive homology of $(X ; *)$ (denoted by $H_{n}^{(*)}(X)$ ).

We directly check that $\partial^{(*)} \partial^{(*)}=0$.

We can put $C_{-1}=\mathbb{Z}$ and $\partial_{0}(x)=1$. We have $\partial_{0} \partial_{1}^{(*)}=0$, so we obtain an augmented distributive chain complex and an augmented (one-term) distributive homology, $\tilde{H}_{n}^{(*)}$. As in the classical case we get:

Proposition 6.2. $H_{n}^{(*)}(X)= \begin{cases}\mathbb{Z} \oplus \tilde{H}_{n}^{(*)}(X) & n=0 \\ \tilde{H}_{n}^{(*)}(X) & \text { otherwise }\end{cases}$

If $(X ; *)$ is a rack then the complex $\left(C_{n}^{(*)}, \partial^{(*)}\right)$ is acyclic, but in the general case of a shelf or spindle homology can be nontrivial with nontrivial free and torsion parts (joint work with A.Crans, K.Putyra and A.Sikora [CPP, $[\mathrm{Pr}-\mathrm{Pu}-1,[\mathrm{P}-\mathrm{S}])$.

If we define $d_{i}: C_{n} \rightarrow C_{n-1}, 0 \leq i \leq n$, by $d_{i}\left(x_{0}, \ldots, x_{n}\right)=$ $\left(x_{0} * x_{i}, \ldots, x_{i-1} * x_{i}, x_{i+1}, \ldots, x_{n}\right)$, then $\left(C_{n}, d_{i}\right)$ is a presimplicial module and $\left.X^{n+1}, d_{i}\right)$ is a presimplicial set. If we define degeneracy maps $s_{i}\left(x_{0}, \ldots, x_{n}\right)=\left(x_{0}, . ., x_{i-1}, x_{i}, x_{i}, x_{i+1}, \ldots x_{n}\right)$ then one checks that $\left(C_{n}, d_{i}, s_{i}\right)$ is a very weak simplicial module. If we assume idempotency, that is $(X ; *)$ is a spindle, then $\left(C_{n}, d_{i}, s_{i}\right)$ is a weak simplicial module and the degenerate part $\left(C_{n}^{D}, \partial_{n}\right)$ is a subchain complex which splits from $C_{n}, \partial_{n}$ ) (see Prz-5]. This split is analogous to the one conjectured in [CJKS] and proved in [L-N] for classical quandle homology. In [N-P-2] we gave very short, easy to visualize and to generalize, proof using the split map $C_{n}^{N} \rightarrow C_{n}$ given by $\left(x_{0}, x_{1}, \ldots, x_{n}\right) \rightarrow$ $\left(x_{0}, x_{1}-x_{0}, \ldots, x_{n}-x_{n-1}\right)$.

We can repeat our definitions if $(X ; *)$ is a shelf and $Y$ is a shelf-set $\left(*: Y \times X \rightarrow Y\right.$ with $\left(y * x_{1}\right) * x_{2}=\left(y * x_{2}\right) *\left(x_{1} * x_{2}\right)$ see Figure 6.1 for visualization). The presimplicial set $\left(Y \times X^{n+1}, d_{i}\right)$ has face maps $d_{i}$ defined by $d_{i}\left(y, x_{0}, \ldots, x_{n}\right)=\left(y * x_{i}, x_{0} * x_{i}, \ldots, x_{i-1} * x_{i}, x_{i+1}, . ., x_{n}\right)$. The face map $d_{i}$ is visualized in Figure 6.2; this visualization will play an important role when distributive homology will be generalized to Yang-Baxter homology. 


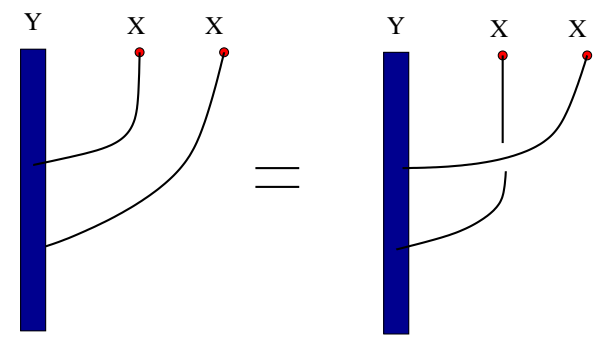

Figure 6.1; Graphical interpretation of the axiom for $X$-shelf-set $Y$ $\left(y * x_{1}\right) * x_{2}=\left(y * x_{2}\right) *\left(x_{1} * x_{2}\right)$

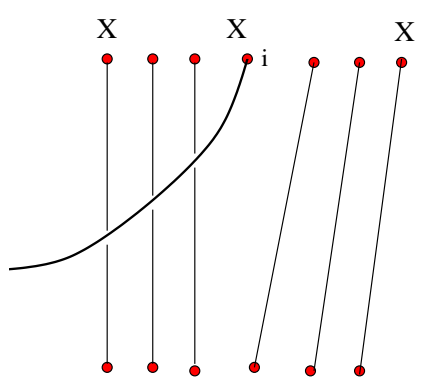

$\mathrm{d}_{\mathrm{i}}^{(*)}$

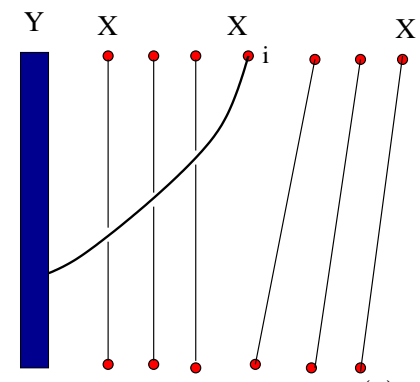

Figure 6.2; Graphical interpretation of the face map $d_{i}^{(*)}$

6.2. Multi-term distributive homology. The first homology theory related to a self-distributive structure was constructed in early 1990s by Fenn, Rourke, and Sanderson [FRS-2 and motivated by (higher dimensional) knot theory 19 . For a rack $(X, *)$, they defined rack homology $H_{n}^{R}(X)$ by taking $C_{n}^{R}=\mathbb{Z} X^{n}$ and $\partial_{n}^{R}: C_{n} \rightarrow C_{n-1}$ is given by $\partial_{n}^{R}=\partial_{n-1}^{(*)}-\partial_{n-1}^{\left(*_{0}\right)}$. Our notation has grading shifted by 1 , that is, $C_{n}(X)=C_{n+1}^{R}=\mathbb{Z} X^{n+1}$. It is routine to check that $\partial_{n-1}^{R} \partial_{n}^{R}=0$. However, it is an interesting question what properties of $*_{0}$ and $*$ are really used. With relation to the paper [N-P-3] we noticed that it is distributivity again which makes $\left(C^{R}(X), \partial_{n}^{R}\right)$ a chain complex. More generally we observed that if $*_{1}$ and $*_{2}$ are right self-distributive and distributive with respect to each other, then $\partial^{\left(a_{1}, a_{2}\right)}=a_{1} \partial^{\left(*_{1}\right)}+a_{2} \partial^{\left(*_{2}\right)}$ leads to a chain complex (i.e. $\left.\partial^{\left(a_{1}, a_{2}\right)} \partial^{\left(a_{1}, a_{2}\right)}=0\right)$. Below I answer a more general question: for a finite set $\left\{*_{1}, \ldots, *_{k}\right\} \subset \operatorname{Bin}(X)$ and integers $a_{1}, \ldots, a_{k} \in$ $\mathbb{Z}$, when is $\left(C_{n}, \partial^{\left(a_{1}, \ldots, a_{k}\right)}\right)$ with $\partial^{\left(a_{1}, \ldots, a_{k}\right)}=a_{1} \partial^{\left(*_{1}\right)}+\ldots+a_{k} \partial^{\left(*_{k}\right)}$ a chain complex? When is $\left(C_{n}, d_{i}^{\left(a_{1}, \ldots, a_{k}\right)}\right)$ a presimplicial set? We answer these

\footnotetext{
${ }^{19}$ The recent paper by Roger Fenn, [Fenn] states: "Unusually in the history of mathematics, the discovery of the homology and classifying space of a rack can be precisely dated to 2 April 1990."
} 
questions in Lemma 6.3. In particular, for a distributive set $\left\{*_{1}, \ldots, *_{k}\right\}$ the answer is affirmative.

\section{Lemma 6.3.}

(i) If $*_{1}$ and $*_{2}$ are right self-distributive operations, then $\left(C_{n}, \partial^{\left(a_{1}, a_{2}\right)}\right)$ is a chain complex if and only if the operations $*_{1}$ and $*_{2}$ satisfy:

\section{4 .}

$\left(a *_{1} b\right) *_{2} c+\left(a *_{2} b\right) *_{1} c=\left(a *_{2} c\right) *_{1}\left(b *_{2} c\right)+\left(a *_{1} c\right) *_{2}\left(b *_{1} c\right)$ in $\mathbb{Z} X$.

We call this condition weak distributivity. If Condition 6.4 does not hold we can take $C_{0}(X)$ to be the quotient by Equation 6.4: $C_{0}(X)=\mathbb{Z} X / \mathbf{6 . 4}$ and then we take $C_{n}=C_{0}^{\otimes n+1}$.

(ii) We say that a set $\left\{*_{1}, \ldots, *_{k}\right\} \subset \operatorname{Bin}(X)$ is weakly distributive if each operation is right self-distributive and each pair of operations is weakly distributive (with two main cases: distributivity $\left(a *_{1} b\right) *_{2} c=\left(a *_{2} c\right) *_{1}\left(b *_{2} c\right)$ and chronological distributivity 20 $\left.\left(a *_{1} b\right) *_{2} c=\left(a *_{1} c\right) *_{2}\left(b *_{1} c\right)\right)$. We have: $\left(C_{n}, d_{i}^{\left(a_{1}, \ldots, a_{k}\right)}\right)$ is a presimplicial set if and only if the set $\left\{*_{1}, \ldots, *_{k}\right\} \subset \operatorname{Bin}(X)$ is weakly distributive.

(iii) $\left(C_{n}, \partial_{n}^{\left(a_{1}, \ldots, a_{k}\right)}\right)$ is a chain complex if and only if the set $\left\{*_{1}, \ldots, *_{k}\right\} \subset$ $\operatorname{Bin}(X)$ is weakly distributive.

We complete this section by showing that for a rack homology of a quandle or spindle, $H_{n}^{R}$ embeds in $H_{n+1}^{R}$; we construct monomorphic "homology operation" of degree on ${ }^{21}$. We place it in a more general context of weak simplicial modules.

Lemma 6.5. Let $\left(C_{n}, d_{i}, s_{i}\right)$ be a weak simplicial module then $\partial s_{0}+$ $s_{0} \partial=s_{0} d_{0}$; in effect $s_{0} d_{0}$ induces a trivial map on homology. In particular:

(i) if the map $s_{0} d_{0}$ is the identity then $s_{0} d_{0} s_{0}=s_{0}$ (as in the case of a simplicial module) and then the chain complex $\left(s_{0}\left(C_{n-1}\right), \partial_{n}\right)$ is acyclic,

(ii) if $d_{0}=0$ then $s_{0}$ is a chain map (e.g. this hold for 2-term rack homology),

(iii) In the case of one term distributive homology, we conclude that the map replacing $x_{0}$ by $x_{1}$ in $\left(x_{0}, x_{1}, \ldots, x_{n}\right)$ is a chain map, chain homotopic to zero map.

\footnotetext{
${ }^{20} \mathrm{I}$ did not see this concept considered in literature, but it seems to be important in K.Putyra's work on odd Khovanov homology Put].

${ }^{21}$ For a quandle it is a well know fact that rack homology in dimension $n$ is isomorphic to "early degenerate" homology in dimension $n+1$.
} 
Proof. We have $\partial s_{0}+s_{0} \partial=$

$$
\begin{gathered}
d_{0} s_{0}-d_{1} s_{0}+\sum_{i=2}^{n+1}(-1)^{i} d_{i} s_{0}+s_{0} d_{0}+\sum_{i=1}^{n}(-1)^{i} s_{0} d_{i}= \\
d_{0} s_{0}-d_{1} s_{0}+\sum_{i=2}^{n+1}(-1)^{i} s_{0} d_{i-1}+s_{0} d_{0}+\sum_{i=1}^{n}(-1)^{i} s_{0} d_{i}=s_{0} d_{0} .
\end{gathered}
$$

If $s_{0}$ has a left inverse map, say $p_{n}: C_{n+1} \rightarrow C_{n}, p_{n} s_{0}=I d_{C_{n}}$, as is the case for a weak simplicial module in (multi) spindle case, we can say more.

Lemma 6.6. Let $\left(C_{n}, d_{i}, s_{i}\right)$ be a weak simplicial module $p_{n}: C_{n+1} \rightarrow$ $C_{n}$ is a left inverse of $s_{0}$ and additionally $p_{n} d_{i}=d_{i-1} p_{n}$ for $i>0$ then $p \partial+\partial p=p d_{0}$; in effect $p d_{0}$ induces a trivial map on homology. In particular:

(i) If we deal with (multi)term distributive homology, $p$ may be taken to be the map deleting the first coordinate of $\left(x_{0}, x_{1}, \ldots, x_{n}\right)$ (in one term distributive homology $p=d_{0}$, so $d_{0} d_{0}$ is a chain map trivial on homology).

(ii) if $d_{0}=0$ then $p$ is a chain map ((e.g. this hold for 2-term rack homology),

(iii) if $d_{0}=0$ then $p$ induces an epimorphism on homology and $s_{0}$ induces a monomorphism on homology; in particular $s_{0}$ induces monomorphic "homology operation" of degree one $\left(s_{0}\left(C_{n-1}, \partial_{n}\right)\right.$ is called an early degenerate chain complex).

Proof. We have $p \partial+\partial p=$

$$
p d_{0}+\sum_{i=1}^{n}(-1)^{i} d_{i-1} p+\sum_{i=0}^{n-1}(-1)^{i} d_{i} p=p d_{0}
$$

Part (iii) follow from the fact that $p_{n} s_{0}=I d_{C_{n}}$ and $p$ and $s_{0}$ are chain maps.

Thus we proved that rack homology of quandles (or spindles) cannot decrease with $n\left(H_{n}^{R} \subset H_{n+1}^{R}\right)$.

We computed with K.Putyra [Pr-Pu-1] various multi-term homology, including that for finite distributive lattices (including Boolean algebras). 


\section{Bloh-Leibniz-Loday Algebra}

Lie algebra was probably the first nonassociative structure for which homology was defined Ch-E. The idea of Chevalley and Eilenberg was to translate homology of a (Lie) group to homology of its Lie algebra 22 . We should stress, in particular the role of conjugacy in Lie algebra, as conjugacy was the motivation for wracks (racks) and quandles.

We discuss here homology theory of Bloh-Loday-Leibniz algebras introduced by Bloh an Loday [Blo-1, Blo-2, Lod-2], and which can be informally thought to be a linearization of distributive homology $23 \mathrm{We}$ follow Loday and Lebed here [Lod-1, Lod-2, Leb-1, Leb-2]. Because BLL (Bloh-Leibniz-Loday) algebra is a generalization of a Lie algebra we use a bracket $[-,-]$ for a bilinear map:

Definition 7.1. (1) Let $V$ be a $k$-module equipped with a bilinear map $[-,-]: V \times V \rightarrow V$ satisfying the relation (Leibniz version of the Jacobi identity):

$$
[x,[y, z]]=[[x, y], z]-[[x, z], y], \text { for all } x, y, z \in V
$$

I see the linearization of distributivity as

$$
(x * y) * z=(x * z) *(y * z) \Longrightarrow(x * y) * z=(x * z) * y+x *(y * z) \text { BLL condition. }
$$

\footnotetext{
${ }^{22}$ The paper starts from: The present paper lays no claim to deep originality. Its main purpose is to give a systematic treatment of the methods by which topological questions concerning compact Lie groups maybe reduced to algebraic questions concerning Lie algebras.

${ }^{23}$ BLL algebras are often call Leibniz algebras as the version of Jacobi identity they satisfy can be treated as a Leibniz rule. The history of the discovery is described by Loday as follows [Lod-1]:

"In the definition of the Chevalley-Eilenberg complex of a Lie algebra $\mathcal{G}$ the module of chains is the exterior module. The non-commutative analog of the exterior module $\Lambda \mathcal{G}$ is the tensor module $T \mathcal{G}$. If one replaces $\Lambda$ by $\bigotimes$ in the classical formula for the boundary map $d$ of the $C E$-complex, then one gets a well defined map $T \mathcal{G}$ but the relation $d^{2}$ is not valid anymore. However I discovered that, if one writes $d$ so as as to put the commutator $\left[x_{i}, x_{j}\right]$ at the place $i$ when $i<j, \ldots$, then the relation $d^{2}=0$ is satisfied in the tensor (i.e. non-commutative) context. So, this give rise to a new complex $T \mathcal{G}, d$ ) for the Lie algebra $\mathcal{G}$. The homology groups of this complex are denoted are denoted $H L_{*}(\mathcal{G})$ and called the non-commutative homology groups of $\mathcal{G}$. In the proof of the relation $d^{2}=0$ in the tensor module case, I noticed that the only property of the Lie bracket, which is needed, is the Leibniz relation $[x,[y, z]]=[[x, y], z]-[[x, z], y]$. So the complex $(T \mathcal{G}, d)$ and its homology are defined for more general objects than Lie algebras, for the Leibniz algebras."
} 
V.Lebed formalized this "linearization" in the case $V$ has a central element 1 that is $[x, 1]=0[1, x]$ we color crossing as follows:<smiles>CC(C)(I)[10I-]</smiles>

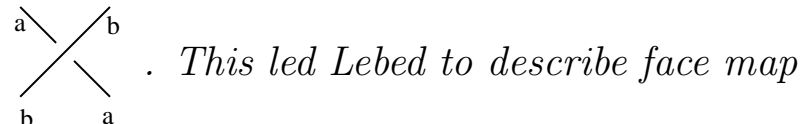
for chain complex of BLL algebras as in Figure 7.1; compare the figure with Definition 7.2.

(2) A BLL module $M$ over $V$ is a $k$-module with a bilinear action (still denoted $[-,-]: M \times V \rightarrow M$, satisfying the formula from (1) for any $x \in M$ and $y, z \in V$.

In a special case of $M=k$ ( $k$ a ring with identity), the map $[-,-]: M \times V \rightarrow M$ is replaced by map $\epsilon: V \rightarrow k$ which is zero on commutators (Lie character), [Leb-2]).
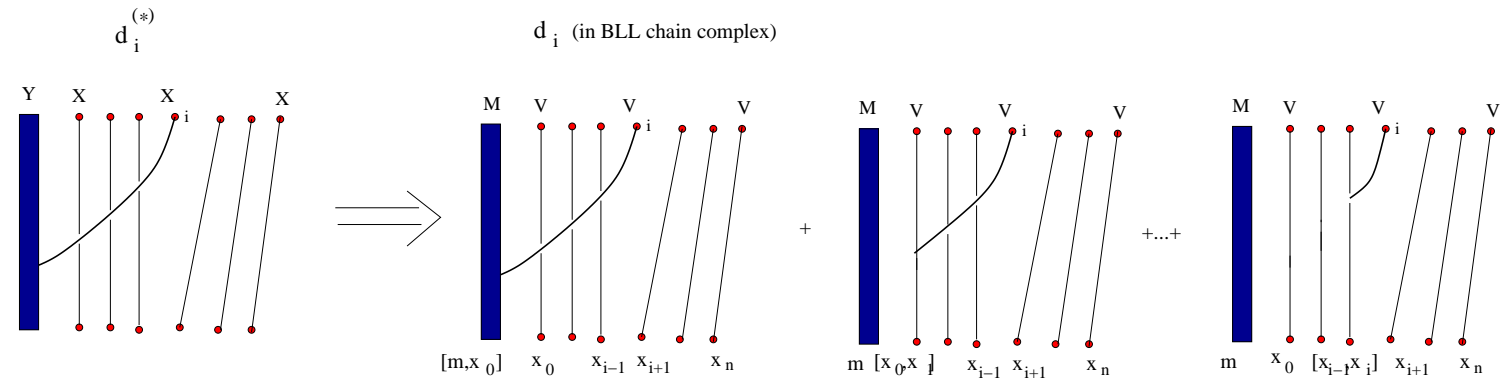

Figure 7.1; Comparing face map $d_{i}$ in distributive and unital BLL algebra

Homology of $(V, M)$ was constructed by Loday based partially on the work of C.Cuvier [Cuv, Lod-1, they were unaware of the earlier work by Bloh [Blo-2.

Definition 7.2. Let $V$ be a BLL algebra, and $M$ a BLL module over $V$.

(1) We define $C_{n}=M \otimes V^{\otimes n+1}$, that is $C_{*}=T V$ (tensor algebra). For $0 \leq i \leq n, d_{i}: C_{n} \rightarrow C_{n-1}$ is given by:

$d_{i}\left(x_{-1}, x_{0}, \ldots, x_{n}\right)=\sum_{j:-1 \leq j<i}\left(x_{-1}, x_{0}, \ldots, x_{j-1},\left[x_{j}, x_{i}\right], x_{j+1}, x_{i-1}, x_{i+1}, \ldots, x_{n}\right)$.

If we take $\Lambda V$, the exterior algebra in place of $T V$. We will be in the classical case of homology developed for Lie algebras by Chevalley and Eilenberg [Ch-E].

(2) Assume that $V$ is a split unital BLL algebra (that is $V=V^{\prime} \oplus k 1$, and $M=k,[1, x]=\epsilon(x)(1 \in k x \in V)$, then the "primitive" 
degenerate maps $s_{i}^{p}: C_{n} \rightarrow C_{n+1}$ is defined by

$s_{i}^{p}\left(x_{-1}, x_{0}, \ldots, x_{n}\right)=\left\{\begin{aligned}\left(x_{-1}, x_{0}, \ldots, x_{i-1},\left(1, x_{i}\right)+\left(x_{i}, 1\right), x_{i+1}, \ldots, x_{n}\right) & \text { if } x_{i} \in V^{\prime} \\ \left(x_{-1}, x_{0}, \ldots, x_{i-1}, 1,1, x_{i+1}, \ldots, x_{n}\right) & \text { if } x_{i}=1\end{aligned}\right.$

(3) Assume $V$ is a free $k$-module with basis $X$ and define degenerate maps $s_{i}^{g}: C_{n} \rightarrow C_{n+1}$ on $X$ by doubling ith coordinate:

$$
s_{i}^{g}\left(m, x_{0}, . ., x_{n}\right)=\left(m, x_{0}, . ., x_{i-1}, x_{i}, x_{i}, x_{i+1}, \ldots, x_{n}\right) .
$$

Lemma 7.3. (1) $\left(C_{n}(V, M), d_{i}\right)$ is a presimplicial module, that is $d_{i} d_{j}=d_{j-1} d_{i}$ for $0 \leq i<j \leq n$.

(2) $\left(C_{n}(X, M), d_{i}, s_{i}^{p}\right)$ is a weak simplicial module, and its homology and degenerated homology are annihilated by $\epsilon(1)$.

(3) $\left(C_{n}(X, M), d_{i}, s_{i}^{g}\right)$ satisfies conditions (1) (2) and partially (3) $\left(d_{i} s_{j}=s_{j-1} d_{i}\right.$ for $\left.i<j\right)$ of a simplicial module. Condition (4'), $d_{i} s_{i}-d_{i+1} s_{i}=0$ holds iff $[x, x]$ for all $x \in X$. The condition (3) of a simplicial module for $i>j+1$ requires the following equality:

$$
\left[x_{j}, x_{i}\right] \otimes\left[x_{j}, x_{i}\right]=\left[x_{j}, x_{i}\right] \otimes x_{j}+x_{j} \otimes\left[x_{j}, x_{i}\right]
$$

for basic elements $\left(x_{j}, x_{i}\right) \in X^{2}$ for $i>j+1$. Thus if we take $C_{*}=V T / \mathcal{I}$ divided by an ideal containing the above equation, then $\left(C_{n}(X, M), d_{i}, s_{i}^{g}\right)$ is a very weak simplicial module. It is a weak simplicial module iff additionally $[x, x]=0$, for all $x \in$ $X 24$

We leave the proof as an exercise for the reader however we make the calculation in two small but typical cases which show that our axioms are needed:

(i) Comparison of $d_{0} d_{1}$ with $d_{0} d_{0}$ (they should be equal):

$d_{0} d_{1}\left(m ; x_{0}, x_{1}\right)=d_{0}\left(\left(\left[m, x_{1}\right] ; x_{0}\right)+\left(m ;\left[x_{0}, x_{1}\right]\right)\right)=\left(\left[m, x_{1}\right], x_{0}\right)+\left[m,\left[x_{0}, x_{1}\right]\right]$,

$\left.\left.d_{0} d_{0}\left(m ; x_{0}, x_{1}\right)=d_{0}\left(\left[m, x_{0}\right] ; x_{1}\right)\right)=\left[\left[m, x_{0}\right)\right], x_{1}\right]$,

Thus $d_{0} d_{1}=d_{0} d_{0}$ if and only if $\left(\left[m, x_{1}\right], x_{0}\right]+\left[m,\left[x_{0}, x_{1}\right]=\left[\left[m, x_{0}\right], x_{1}\right]\right.$

which is the axiom of BLL-module.

(ii) Comparison of $d_{1} d_{2}$ with $d_{1} d_{1}$ (they should be equal):

$d_{1} d_{2}\left(m ; x_{0}, x_{1}, x_{2}\right)=d_{1}\left(\left(\left[m, x_{2}\right] ; x_{0}, x_{1}\right)+\left(m ;\left[x_{0}, x_{2}\right], x_{1}\right)+\left(m ; x_{0},\left[x_{1}, x_{2}\right]\right)\right)=$

$\left(\left[\left[m, x_{2}\right], x_{1}\right] ; x_{0}\right)+\left(\left(\left[m, x_{2}\right] ;\left[x_{0}, x_{1}\right]\right)+\right.$

$\left(\left[m, x_{1}\right] ;\left[x_{0}, x_{2}\right]\right)+\left(m ;\left[\left[x_{0}, x_{2}\right], x_{1}\right]\right)+$

$\left(\left[m,\left[x_{1}, x_{2}\right]\right] ; x_{0}\right)+\left(m ;\left[x_{0},\left[x_{1}, x_{2}\right]\right]\right)$

and

$\left.d_{1} d_{1}\left(m ; x_{0}, x_{1}, x_{2}\right)=d_{1}\left(\left[m, x_{1}\right] ; x_{0}, x_{2}\right)+\left(m ;\left[x_{0}, x_{1}\right], x_{2}\right)\right)=$

\footnotetext{
${ }^{24}$ Notice that in exterior algebra $\Lambda V$, the equation $\left[x_{j}, x_{i}\right] \otimes\left[x_{j}, x_{i}\right]=\left[x_{j}, x_{i}\right] \otimes$ $x_{j}+x_{j} \otimes\left[x_{j}, x_{i}\right]$ holds $(0=0)$, but in that case our degenerate map $s_{i}^{g}$ would be a zero map.
} 
$\left(\left[\left[m, x_{1}\right], x_{2}\right] ; x_{0}\right)+\left(\left[m, x_{1}\right] ;\left[x_{0}, x_{2}\right]\right)+$ $\left(\left[m, x_{2}\right] ;\left[x_{0}, x_{1}\right]\right)+\left(m ;\left[\left[x_{0}, x_{1}\right], x_{2}\right]\right)$.

Thus $d_{1} d_{2}=d_{1} d_{1}$ if and only if the following sum is equal to zero:

$$
\begin{gathered}
\left(\left[\left[m, x_{2}\right], x_{1}\right] ; x_{0}\right)+\left(\left[m,\left[x_{1}, x_{2}\right]\right] ; x_{0}\right)-\left(\left[\left[m, x_{1}\right], x_{2}\right] ; x_{0}\right)+ \\
\left(m ;\left[\left[x_{0}, x_{2}\right], x_{1}\right]\right)+\left(m ;\left[x_{0},\left[x_{1}, x_{2}\right]\right]\right)-\left(m ;\left[\left[x_{0}, x_{1}\right], x_{2}\right]\right) .
\end{gathered}
$$

The first part is equal to zero iff $M$ is BLL-module and the second part is equal to zero iff $V$ is BLL-algebra.

Remark 7.4. Lie algebra homology, as proved by Cartan and Eilenberg [Ca-E] can be obtained from homology of the universal enveloping algebra $U V=T V /(a \otimes b-b \otimes a=[a, b])$ of the Lie algebra $V$. One hopes for a similar connection between distributive homology and homology of the group associated to a wrack or quandle. One hint in this direction is that in every group the following "distributivity" holds:

$$
\left[\left[x, y^{-1}\right], z\right]^{y}\left[\left[y, z^{-1}\right], x\right]^{z}\left[\left[z, x^{-1}\right], y\right]^{x}=1 \text {, }
$$

where $[x, y]=x^{-1} y^{-1} x y$ and $x^{y}=y^{-1} x y$. This leads to the graded Lie algebra associated to the group, via lower central series of the group [Va].

\section{Semigroup EXTENSIONS AND SHELF EXTENSIONS}

The theory of extension of structures and related cocycles started from two important examples from group theory:

(i) The extension of $P S L_{n}(C)$ by $S L_{n}(C)$ by I.Schur (1904), with related short exact sequence of groups [B-T]

$$
0 \rightarrow \mathbb{Z}_{2} \rightarrow S L_{n}(C) \rightarrow P S L_{n}(C) \rightarrow 1 \text { and }
$$

the study of crystallographic groups $\Gamma$ where we consider a short exact sequence

$$
0 \rightarrow \mathbb{Z}^{n} \rightarrow \Gamma \rightarrow \Gamma / \mathbb{Z}^{n} \rightarrow 1
$$

An extension of a group $X$ by a group $N$ is a short exact sequence of groups

$$
1 \rightarrow N \stackrel{i}{\rightarrow} E \stackrel{\pi}{\rightarrow} X \rightarrow 1
$$

(some people call this an extension of $N$ by $X$ [B-T, Bro, Ma-Bi]). Consider a set-theoretic section $s: X \rightarrow E$ (that is $\left.\pi s=I d_{X}\right)$. Every element of $E$ is a unique product $a s(x)$ for $a \in N$ and $x \in X$ (coset decomposition), thus we have $E=N \times X$ as sets (here $e \rightarrow$ $\left(e s\left(\pi\left(e^{-1}\right)\right), \pi(e)\right)$ and $e s\left(\pi\left(e^{-1}\right)\right)(s \pi(e))=e$ as needed. The inverse map is $(a, x) \rightarrow a s(x)$.

This motivates study of extension of magmas as study of projections $\pi: A \times X \rightarrow X$ with various structures preserved. In particular, 
we compare semigroup extension of a semigroup by an abelian group with the shelf extension of a shelf by an Alexander quandle. We start from a general concept of a dynamic cocycle in a magma case and then in associative and distributive cases and in both we relate to the (co)homology of our structures. Extension of modules, groups and Lie algebras is described in the classical book by Cartan and Eilenberg Ca-E], distributive case was developed in [CES-2, A-G, CKS].

Definition 8.1. Let $(X ; *)$ be a magma, $A$ a set, and $\pi: A \times X \rightarrow X$ the projection to the second coordinate. Any magma structure on $A \times X$ for which $\pi$ is an epimorphism, can be given by a system of functions $\phi_{a_{1}, a_{2}}\left(x_{1}, x_{2}\right): X \times X \rightarrow A$ by:

$$
\left(a_{1}, x_{1}\right) *\left(a_{2}, x_{2}\right)=\left(\phi_{a_{1}, a_{2}}\left(x_{1}, x_{2}\right), x_{1} * x_{2}\right) .
$$

Functions $\phi_{a_{1}, a_{2}}\left(x_{1}, x_{2}\right)$ are uniquely defined by the multiplication on $A \times X$, thus binary operations on $A \times X$ agreeing with $\pi$ are in bijection with choices of functions $\phi_{a_{1}, a_{2}}$. If we require some special structure on $(X ; *)$ (e.g. associativity or right-distributivity) we obtain some property of $\phi_{a_{1}, a_{2}}\left(x_{1}, x_{2}\right)$ which we call a dynamical co-cycle property for the structure.

(1) Let $(X ; *)$ be a semigroup; in order that an action on $A \times X$ is associative we need:

$$
\begin{gathered}
\left(\left(a_{1}, x_{1}\right) *\left(a_{2}, x_{2}\right)\right) *\left(a_{3}, x_{3}\right)=\left(\phi_{a_{1}, a_{2}}\left(x_{1}, x_{2}\right), x_{1} * x_{2}\right) *\left(a_{3}, x_{3}\right)= \\
\left(\phi_{\phi_{a_{1}, a_{2}}\left(x_{1}, x_{2}\right), a_{3}}\left(x_{1} * x_{2}, x_{3}\right),\left(x_{1} * x_{2}\right) * x_{3}\right)
\end{gathered}
$$

to be equal to

$$
\begin{gathered}
\left.\left(a_{1}, x_{1}\right) *\left(\left(a_{2}, x_{2}\right)\right) *\left(a_{2}, x_{3}\right)\right)=\left(a_{1}, x_{1}\right) *\left(\phi_{a_{2}, a_{3}}\left(x_{2}, x_{3}\right), x_{2} * x_{3}\right)= \\
\left.\left(\phi_{a_{1}, \phi_{a_{2}, a_{3}}}\left(x_{2}, x_{3}\right)\right)\left(x_{1}, x_{2} * x_{3}\right), x_{1} *\left(x_{2} * x_{3}\right)\right) .
\end{gathered}
$$

Thus the dynamical cocycle condition in the associative case has a form:

$$
\left(\phi_{\phi_{a_{1}, a_{2}}\left(x_{1}, x_{2}\right), a_{3}}\left(x_{1} * x_{2}, x_{3}\right)=\phi_{a_{1}, \phi_{a_{2}, a_{3}}\left(x_{2}, x_{3}\right)}\left(x_{1}, x_{2} * x_{3}\right)\right) .
$$

(2) Let $(X ; *)$ be a shelf; in order that an action on $A \times X$ is right self-distributive we need:

$$
\begin{gathered}
\left(\left(a_{1}, x_{1}\right) *\left(a_{2}, x_{2}\right)\right) *\left(a_{2}, x_{3}\right)=\left(\phi_{a_{1}, a_{2}}\left(x_{1}, x_{2}\right), x_{1} * x_{2}\right) *\left(a_{3}, x_{3}\right)= \\
\left(\phi_{\left.\phi_{a_{1}, a_{2}}\left(x_{1}, x_{2}\right), a_{3}\right)}\left(x_{1} * x_{2}, x_{3}\right),\left(x_{1} * x_{2}\right) * x_{3}\right)
\end{gathered}
$$

to be equal to

$$
\begin{gathered}
\left(\left(a_{1}, x_{1}\right) *\left(a_{3}, x_{3}\right)\right) *\left(\left(a_{2}, x_{2}\right) *\left(a_{3}, x_{3}\right)\right)= \\
\left(\phi_{a_{1}, a_{3}}\left(x_{1}, x_{3}\right), x_{1} * x_{3}\right) *\left(\phi_{a_{2}, a_{3}}\left(x_{2}, x_{3}\right), x_{2} * x_{3}\right)= \\
\left(\phi_{\phi_{a_{1}, a_{3}}\left(x_{1}, x_{3}\right), \phi_{a_{2}, a_{3}}\left(x_{2}, x_{3}\right)}\left(x_{1} * x_{3}, x_{2} * x_{3}\right),\left(x_{1} * x_{3}\right) *\left(x_{2} * x_{3}\right)\right) .
\end{gathered}
$$


Thus the dynamical cocycle condition in right-distributive case has a form:

$\phi_{\phi_{a_{1}, a_{2}}\left(x_{1}, x_{2}\right), a_{3}}\left(x_{1} * x_{2}, x_{3}\right)=\phi_{\phi_{a_{1}, a_{3}}\left(x_{1}, x_{3}\right), \phi_{a_{2}, a_{3}}\left(x_{2}, x_{3}\right)}\left(x_{1} * x_{3}, x_{2} * x_{3}\right)$.

(3) We assume now that $(X ; *)$ is an entropic (inner turn) magma, that is $(a * b) *(c * d)=(a * c) *(b * d)$ for any $a, b, c, d \in X$. $W e$ look for condition on the dynamical co-cycle so that $A \times X$ is entropic. We need

$$
\begin{gathered}
\left(\left(a_{1}, x_{1}\right) *\left(a_{2}, x_{2}\right)\right) *\left(\left(a_{3}, x_{3}\right) *\left(a_{4}, x_{4}\right)\right)= \\
\left(\phi_{a_{1}, a_{2}}\left(x_{1}, x_{2}\right), x_{1} * x_{2}\right) *\left(\phi_{a_{3}, a_{4}}\left(x_{3}, x_{4}\right), x_{3} * x_{4}\right)= \\
\left(\phi_{\phi_{a_{1}, a_{2}}\left(x_{1}, x_{2}\right), \phi_{a_{3}, a_{4}}\left(x_{3}, x_{4}\right)}\left(x_{1} * x_{2}, x_{3} * x_{4}\right),\left(x_{1} * x_{2}\right) *\left(x_{3} * x_{4}\right)\right)
\end{gathered}
$$

to be equal to

$$
\begin{gathered}
\left(\left(a_{1}, x_{1}\right) *\left(a_{3}, x_{3}\right)\right) *\left(\left(a_{2}, x_{2}\right) *\left(a_{3}, x_{3}\right)\right)= \\
\left(\phi_{a_{1}, a_{3}}\left(x_{1}, x_{3}\right), x_{1} * x_{3}\right) *\left(\phi_{a_{2}, a_{4}}\left(x_{2}, x_{4}\right), x_{2} * x_{4}\right)= \\
\left(\phi_{\phi_{a_{1}, a_{3}}\left(x_{1}, x_{3}\right), \phi_{a_{2}, a_{4}}\left(x_{2}, x_{4}\right)}\left(x_{1} * x_{3}, x_{2} * x_{4}\right),\left(x_{1} * x_{3}\right) *\left(x_{2} * x_{4}\right)\right) .
\end{gathered}
$$

Thus the dynamic cocycle condition in entropic case has the form:

$\phi_{\phi_{a_{1}, a_{2}}\left(x_{1}, x_{2}\right), \phi_{a_{3}, a_{4}}\left(x_{3}, x_{4}\right)}\left(x_{1} * x_{2}, x_{3} * x_{4}\right)=\phi_{\phi_{a_{1}, a_{3}}\left(x_{1}, x_{3}\right), \phi_{a_{2}, a_{4}}\left(x_{2}, x_{4}\right)}\left(x_{1} * x_{3}, x_{2} * x_{4}\right)$.

We illustrate the above by several examples, starting from a classical group extension by an abelian group. Consider the extension $E$ of a group $X$ by an abelian group $A$; this is described by a short exact sequence of groups:

$$
0 \rightarrow A \rightarrow E \stackrel{\pi}{\rightarrow} X \rightarrow 1
$$

As noted before, $E=A \times X$ as a set and bijection depends on a section $s: X \rightarrow E$. Furthermore $X$ acts on $A$ (we have $X \times A \rightarrow A$ ) and the action is given by conjugation: $x(a)=s(x) a\left(s(x)^{-1}\right.$ and does not depend on the choice of $s$ as $A$ is commutative).

For a semigroup this is the starting point.

Let $X$ be a semigroup, $\pi: A \times X$ a projection and a semigroup $X$ act on a set $A$. We define a product on $A \times X$ by the formula:

$$
\left(a_{1}, x_{1}\right)\left(a_{2}, x_{2}\right)=\left(a_{1}+x_{1}\left(a_{2}\right)+f\left(x_{1}, x_{2}\right), x_{1} x_{2}\right) .
$$

The function $f: X \times X \rightarrow A$, as in the group case, arise by comparing section of a multiplication with multiplication of sections, that is $s\left(x_{1} x_{2}\right)=f\left(x_{1}, x_{2}\right) s\left(x_{1}\right) s\left(x_{2}\right)$. We assume that the action $x$ : $A \rightarrow A$ is a group homomorphism for any $x$ and it is associative $\left(x_{1}\left(x_{2}(a)=\left(x_{1} x_{2}\right)(a)\right)\right.$. The associativity of the product on $A \times X$ 
is equivalent to the condition on $f: X \times X \rightarrow A$ of the form 25: $x_{1}\left(f\left(x_{2}, x_{3}\right)\right)-f\left(x_{1} x_{2}, x_{3}\right)+f\left(x_{1}, x_{2} x_{3}\right)-f\left(x_{1}, x_{2}\right)=0$ which we call a second cocycle condition (relation to homology of groups defined before, will be explained). Thus $\phi_{a_{1}, a_{2}}\left(x_{1}, x_{2}\right)=a_{1}+x_{1}\left(a_{2}\right)+f\left(x_{1}, x_{2}\right)$, is an example of a dynamical cocycle for an associative structure. We should stress that for a semigroup there may be choice for a dynamical cocycle but for a group it is unique (see e.g. [Bro]). $f: X \times X \rightarrow A$ is a cocycle for a chain complex introduced in Definition 4.1 for the trivial action (and generally Definition 4.2); we have:

$$
\begin{gathered}
\partial^{2}(f)\left(x_{1}, x_{2}, x_{3}\right)= \\
f\left(\partial_{2}\left(\left(x_{1}, x_{2}, x_{3}\right)\right)=f\left(\left(x_{2}, x_{3}\right)-\left(x_{1} x_{2}, x_{3}\right)+\left(x_{1}, x_{2} x_{3}\right)-\left(x_{1}, x_{2}\right)\right)=\right. \\
f\left(x_{2}, x_{3}\right)-\left(x_{1} x_{2}, x_{3}\right)+f\left(x_{1}, x_{2} x_{3}\right)-f\left(x_{1}, x_{2}\right)=0 .
\end{gathered}
$$

If action of $X$ on $A$ is not necessarily trivial, we define cohomology $H^{n}(G, C)$ with a cochain complex $C_{n}=\operatorname{Hom}\left(Z G^{n} \rightarrow A\right)$ and $\partial^{n}: C^{n} \rightarrow C^{n+1}$ is given by $\partial^{n}(f)\left(x_{1}, \ldots, x_{n}, x_{n+1}\right)=$ $x_{1} f\left(x_{2}, \ldots, x_{n+1}\right)+\sum_{i=1}^{n}(-1)^{i} f\left(x_{1}, \ldots, x_{i} x_{i+1}, \ldots x_{n+1}\right)+(-1)^{n+1}\left(x_{1}, \ldots, x_{n}\right)$.

8.1. Extensions in right distributive case. We give here two examples of extension in right distributive case:

I. Let $(X ; *)$ be a shelf and $A$ an abelian group with a given homomorphism $t: A \rightarrow A$ (equivalently, $A$ is a $\mathbb{Z}[t]$ module). We define a shelf structure (called Alexander extension [CES-2, CKS]) on $A \times X$ by the formula:

$$
\left(a_{1}, x_{1}\right)\left(a_{2}, x_{2}\right)=\left(t a_{1}+(1-t) a_{2}+f\left(x_{1}, x_{2}\right), x_{1} * x_{2}\right)
$$

and right distributivity is equivalent to the condition on $f: X \times X \rightarrow A$ which satisfies twisted cocycle condition:

$t\left(f\left(x_{2}, x_{3}\right)-f\left(x_{1}, x_{3}\right)+f\left(x_{1}, x_{2}\right)\right)-f\left(x_{2}, x_{3}\right)+f\left(x_{1} * x_{2}, x_{3}\right)-f\left(x_{1} * x_{3}, x_{2} * x_{3}\right)=0$.

\footnotetext{
${ }^{25}$ Calculation is as follows: Associativity,

$\left.\left(\left(a_{1}, x_{1}\right)\left(a_{2}, x_{2}\right)\right)\left(a_{3}, x_{3}\right)=\left(a_{1}, x_{1}\right)\left(\left(a_{2}, x_{2}\right)\right)\left(a_{3}, x_{3}\right)\right)$ gives, after expanding each side:$$
\left(\left(a_{1}, x_{1}\right)\left(a_{2}, x_{2}\right)\right)\left(a_{3}, x_{3}\right)=\left(a_{1}+x_{1}\left(a_{2}\right)+f\left(x_{1}, x_{2}\right), x_{1} x_{2}\right)\left(a_{3}, x_{3}\right)=
$$$$
\left(a_{1}+x_{1}\left(a_{2}\right)+f\left(x_{1}, x_{2}\right)+\left(x_{1} x_{2}\right)\left(a_{3}\right)+f\left(x_{1} x_{2}, x_{3}\right),\left(x_{1} x_{2}\right) x_{3}\right) \text { and }
$$$$
\left.\left(a_{1}, x_{1}\right)\left(\left(a_{2}, x_{2}\right)\right)\left(a_{3}, x_{3}\right)\right)=\left(a_{1}, x_{1}\right)\left(a_{2}+x_{2}\left(a_{3}\right)+f\left(x_{2}, x_{3}\right), x_{2} x_{3}\right)=
$$$$
\left(a_{1}+x_{1}\left(a_{2}+x_{2}\left(a_{3}\right)+f\left(x_{2}, x_{3}\right)+f\left(x_{1}, x_{2} x_{3}\right), x_{1}\left(x_{2} x_{3}\right)\right)\right.
$$

thus the associativity reduces to:

$f\left(x_{1}, x_{2}\right)+f\left(x_{1} x_{2}, x_{3}\right)=x_{1}\left(f\left(x_{2}, x_{3}\right)\right)+f\left(x_{1}, x_{2} x_{3}\right)$ which is our 2-cocycle condition. 
The calculation is as follows: Right self-distributivity

$$
\left(\left(a_{1}, x_{1}\right) *\left(a_{2}, x_{2}\right)\right) *\left(a_{3}, x_{3}\right)=\left(\left(a_{1}, x_{1}\right) *\left(a_{3}, x_{3}\right)\right) *\left(\left(a_{2}, x_{2}\right) *\left(a_{3}, x_{3}\right)\right)
$$

gives, after expanding each side:

$$
\begin{gathered}
\left(\left(a_{1}, x_{1}\right) *\left(a_{2}, x_{2}\right)\right) *\left(a_{3}, x_{3}\right)=\left(t a_{1}+(1-t) a_{2}+f\left(x_{1}, x_{2}\right), x_{1} * x_{2}\right) *\left(a_{3}, x_{3}\right)= \\
\left(t\left(t a_{1}+(1-t) a_{2}+f\left(x_{1}, x_{2}\right)\right)+(1-t) a_{3}+f\left(x_{1} * x_{2}, x_{3}\right),\left(x_{1} * x_{2}\right) * x_{3}\right) \text { and } \\
\left(\left(a_{1}, x_{1}\right) *\left(a_{3}, x_{3}\right)\right) *\left(\left(a_{2}, x_{2}\right) *\left(a_{3}, x_{3}\right)\right)= \\
\left(t a_{1}+(1-t) a_{3}+f\left(x_{1}, x_{3}\right), x_{1} * x_{3}\right) *\left(t a_{2}+(1-t) a_{3}+f\left(x_{2}, x_{3}\right), x_{2} * x_{3}\right)= \\
\left(t\left(t a_{1}+(1-t) a_{3}+f\left(x_{1}, x_{3}\right)\right)+(1-t)\left(t a_{2}+(1-t) a_{3}+\right.\right. \\
\left.\left.f\left(x_{2}, x_{3}\right)\right)+f\left(x_{1} * x_{3}, x_{2} * x_{3}\right),\left(x_{1} * x_{3}\right) *\left(x_{2} * x_{3}\right)\right)
\end{gathered}
$$

This is equivalent to:

$\left.t f\left(x_{1}, x_{2}\right)+f\left(x_{1} * x_{2}, x_{3}\right)=t f\left(x_{1}, x_{3}\right)+(1-t) f\left(x_{2}, x_{3}\right)\right)+f\left(x_{1} * x_{3}, x_{2} * x_{3}\right)$, and further to a cocycle in a (twisted) rack homology:

$$
\begin{gathered}
\left(\partial^{R} f\right)\left(x_{1}, x_{2}, x_{3}\right)= \\
\left.-t\left(f\left(x_{2}, x_{3}\right)-f\left(x_{1}, x_{3}\right)\right)+f\left(x_{1}, x_{2}\right)\right)+ \\
f\left(x_{2}, x_{3}\right)-f\left(x_{1} * x_{2}, x_{3}\right)+f\left(x_{1} * x_{3}, x_{2} * x_{3}\right)=0 .
\end{gathered}
$$

If there are two right self-distributive binary operations, $*_{1}$ and $*_{2}$ on $A \times X$ represented by $f_{1}$ and $f_{2}$ respectively (that is $\left(a_{1}, x_{1}\right) *_{i}\left(a_{2}, x_{2}\right)=$ $\left(a_{1} * a_{2}+f_{i}\left(x_{1}, x_{2}\right), x_{1} * x_{2}, i=1,2\right)$, and there is a homomorphism $H: A \times X \rightarrow A \times X$ given by $H(a, x)=(a+c(x), x)$ for some $c: X \rightarrow A$ then the homomorphism condition

$H\left(\left(a_{1}, x_{1}\right) *_{1}\left(a_{2}, x_{2}\right)\right)=H\left(a_{1}, x_{1}\right) *_{2} H\left(a_{2}, x_{2}\right)$ is equivalent to

$$
\left(a_{1} * a_{2}+f_{1}\left(x_{1}, x_{2}\right)+c\left(x_{1} * x_{2}\right), x_{1} * x_{2}\right)=
$$

$$
\left(\left(a_{1}+c\left(x_{1}\right)\right) *\left(a_{2}+c\left(x_{2}\right)\right)+f_{2}\left(x_{1}, x_{2}\right), x_{1} * x_{2}\right) \text { thus }
$$

$t a_{1}+(1-t) a_{2}+c\left(x_{1} * x_{2}\right)+f_{1}\left(x_{1}, x_{2}\right)=t\left(a_{1}+c\left(x_{1}\right)+(1-t)\left(a_{2}+c\left(x_{2}\right)\right)+\right.$ $f_{2}\left(x_{1}, x_{2}\right)$ so $f_{1}\left(x_{1}, x_{2}\right)-f_{2}\left(x_{1}, x_{2}\right)=t c\left(x_{1}\right)+(1-t) c\left(x_{2}\right)-c\left(x_{1} x_{2}\right)=$ $(\partial c)\left(x_{1}, x_{2}\right)$. We can say that the second cohomology (here (twisted) rack cohomology) $H^{2}(X, A)$ describes (shelf) extensions of $X$ by $A$ of type described above, modulo described above equivalence, for an abelian group $A$.

The dynamical cocycle is given by $\phi_{a_{1}, a_{2}}\left(x_{1}, x_{2}\right)=t a_{1}+(1-t) a_{2}+$ $f\left(x_{1}, x_{2}\right)$, CKS.

II. Another family of extensions is given by the hull construction for a multi-shelf (multi-RD-system) of Patrick Dehornoy and David Larue Deh-2, Lar], and its "G-group" generalization (which we call a twisted hull) by Ishii, Iwakiri, Jang, and Oshiro [IIJO].

For a hull construction we need a distributive set of binary operations 
on $A$ indexed by elements of $X$, that is $\left(a *_{x} b\right) *_{y} c=\left(a *_{y} c\right) *_{x}\left(b *_{y} c\right)$, and the "hull" shelf structure on $A \times X$ is given by:

$$
\left(a_{1}, x_{1}\right) *\left(a_{2}, x_{2}\right)=\left(a_{1} *_{x_{2}} a_{2}, x_{1}\right)
$$

To see our construction as obtained from a dynamical cocycle we put trivial operation on $X(x * y=x)$, and the dynamical cocycle is given by $\phi_{a_{1}, a_{2}}\left(x_{1}, x_{2}\right)=a_{1} *_{x_{2}} a_{2}$.

Remark 8.2. If $A=F(X)$ is a fee group on free generators $X$, then the hull $A \times X$ related to the distributive set of operations $*_{x}$ on $A$ given by $a_{1} *_{x} a_{2}=a_{1} a_{2}^{-1} x a_{2}$ is a free rack generated by $X$ (denoted by $F R(X))$ as defined by Fenn and Rourke [F-R] (see also [CKS]). To summarize, we have then $F R(X)=F(X) \times X$ with $\left(a_{1}, x_{1}\right) *\left(a_{2}, x_{2}\right)=$ $\left(a_{1} *_{x_{2}} a_{2}, x_{1}\right)=\left(a_{1} a_{2}^{-1} x_{2} a_{2}, x_{1}\right)$.

The $G$-group generalization of hull to twisted hull, relaxes condition that $X$ is indexing distributive set of operation and we allow "twisted distributivity". That is: $\left(a *_{x} b\right) *_{y} c=\left(a *_{y} c\right) *_{x * y}\left(b *_{y} c\right)$. Thus $X$ indexes operations on $A$ satisfying "twisted distributivity". In this case the shelf structure on $A \times X$ is given by:

$$
\left(a_{1}, x_{1}\right) *\left(a_{2}, x_{2}\right)=\left(a_{1} *_{x_{2}} a_{2}, x_{1} * x_{2}\right) .
$$

The fundamental example leading to "twisted distributivity" was already given by Joyce: Let $G$ be a group and $X$ be a subgroup of $\operatorname{hom}(G, G)$. Then we define $g_{1} *_{x} g_{2}=x\left(g_{1} g_{2}^{-1}\right) g_{2}$ and we get:

$$
\left(g_{1} *_{x_{2}} g_{2}\right) *_{x_{3}} g_{3}=\left(g_{1} *_{x_{3}} g_{3}\right) *_{x_{2} * x_{3}}\left(g_{2} *_{x_{3}} g_{3}\right)
$$

where $x_{2} * x_{3}=x_{3} x_{2} x_{3}^{-1}$.

Twisted distributivity is illustrated in Figure 8.1 below: 


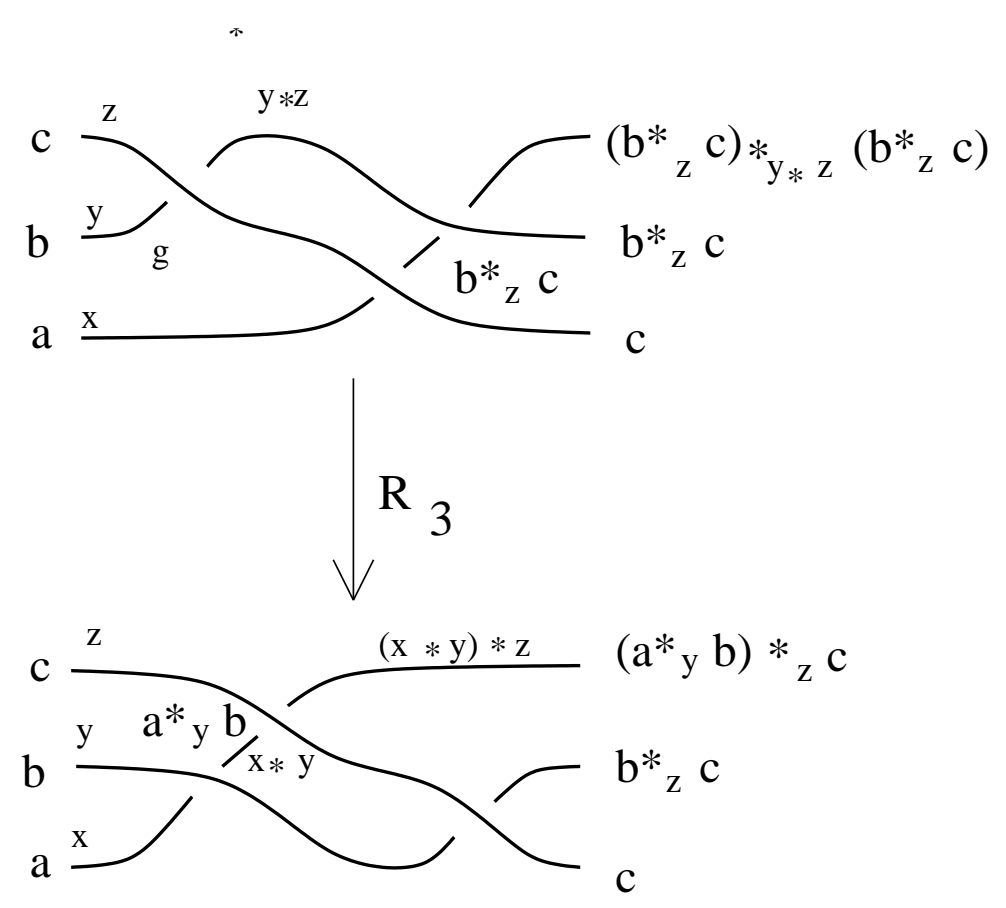

Figure 8.1; Twisted distributivity

8.2. Extensions in entropic case. Let $(X ; *)$ be an entropic magma, that is $*$ satisfies the entropic identity: $(a * b) *(c * d)=(a * c) *(b *$ $d)$. Let also $A$ be an abelian group with a given pair of commuting homomorphisms $t, s: A \rightarrow A$ and a constant $a_{0} \in A$; we consider $(A ; *)$ as an entropic magma with an affine action $a * b=t a+s b+a_{0}$. Then we define a binary operation on $A \times X$ by $\left(a_{1} * x_{1}\right) *\left(a_{2} * x_{2}\right)=\left(a_{1} *\right.$ $\left.a_{2}+f\left(x_{1}, x_{2}\right), x_{1} * x_{2}\right)$. In order for $A \times X$ to be entropic magma we need entropic condition, or equivalently $\phi_{a_{1}, a_{2}}\left(x_{1}, x_{2}\right)=a_{1} * a_{2}+f\left(x_{1}, x_{2}\right)$ should be and entropic dynamic cocycle. This leads to entropic cocycle condition:

$t f\left(x_{1}, x_{2}\right)-t f\left(x_{1}, x_{3}\right)+s f\left(x_{3}, x_{4}\right)-s f\left(x_{2}, x_{4}\right)+f\left(x_{1} * x_{2}, x_{3} * x_{4}\right)-f\left(x_{1} * x_{3}, x_{2} * x_{4}\right)=026$

\footnotetext{
${ }^{26}$ Calculation is as follows: Entropic condition, $\left(\left(a_{1}, x_{1}\right) *\left(a_{2}, x_{2}\right)\right) *\left(\left(a_{3}, x_{3}\right) *\right.$ $\left.\left(a_{4}, x_{4}\right)\right)=\left(\left(a_{1}, x_{1}\right) *\left(a_{3}, x_{3}\right)\right) *\left(\left(a_{2}, x_{2}\right) *\left(a_{4}, x_{4}\right)\right)$ gives, after expanding each side: $\left(\left(a_{1}, x_{1}\right) *\left(a_{2}, x_{2}\right)\right) *\left(\left(a_{3}, x_{3}\right) *\left(a_{4}, x_{4}\right)\right)=\left(a_{1} * a_{2}+f\left(x_{1}, x_{2}\right), x_{1} * x_{2}\right) *\left(a_{3} * a_{4}+\right.$ $\left.f\left(x_{3}, x_{4}\right), x_{3} * x_{4}\right)=\left(\left(a_{1} * a_{2}\right) *\left(a_{3} * a_{4}\right)+t f\left(x_{1}, x_{2}\right)+s f\left(x_{3}, x_{4}\right)+f\left(x_{1} * x_{2}, x_{3} *\right.\right.$ $\left.\left.x_{4}\right),\left(x_{1} * x_{2}\right) *\left(x_{3} * x_{4}\right)\right)$

Similarly $\left(\left(a_{1}, x_{1}\right) *\left(a_{3}, x_{3}\right)\right) *\left(\left(a_{2}, x_{2}\right) *\left(a_{4}, x_{4}\right)\right)=\left(\left(a_{1} * a_{3}\right) *\left(a_{2} * a_{4}\right)+t f\left(x_{1}, x_{3}\right)+\right.$ $\left.s f\left(x_{2}, x_{4}\right)+f\left(x_{1} * x_{3}, x_{2} * x_{4}\right),\left(x_{1} * x_{3}\right) *\left(x_{2} * x_{4}\right)\right)$, which reduces to (entropic) cocycle condition $t f\left(x_{1}, x_{2}\right)+s f\left(x_{3}, x_{4}\right)+f\left(x_{1} * x_{2}, x_{3} * x_{4}\right)=t f\left(x_{1}, x_{3}\right)+s f\left(x_{2}, x_{4}\right)+$ $\left(x_{1} * x_{3}, x_{2} * x_{4}\right)$.
} 
The above formula may serve as a hint how to define (co)homology in entropic case [N-P-4].

In particular $\partial: R X^{4} \rightarrow R X^{2}$ may be given by:

$$
\partial\left(x_{1}, x_{2}, x_{3}, x_{4}\right)=
$$

$t\left(x_{1}, x_{2}\right)-t\left(x_{1}, x_{3}\right)+s\left(x_{3}, x_{4}\right)-s\left(x_{2}, x_{4}\right)+\left(x_{1} * x_{2}, x_{3} * x_{4}\right)-\left(x_{1} * x_{3}, x_{2} * x_{4}\right)$

and $\partial: R X^{2} \rightarrow R X$ may be given by: $\partial\left(x_{1}, x_{2}\right)=t x_{1}-x_{1} * x_{2}+s x_{2}$ (here it agrees with the rack case for $s=1-t$ ).

Remark 8.3. An important, but not yet fully utilized, observation from [N-P-4], is that we can consider atomic boundary functions

$\partial^{(*)}\left(\left(x_{1}, x_{2}, x_{3}, x_{4}\right)=\left(x_{1} * x_{2}, x_{3} * x_{4}\right)-\left(x_{1} * x_{3}, x_{2} * x_{4}\right)\right.$ and $\partial^{(*)}\left(x_{1}, x_{2}\right)=$ $-x_{1} * x_{2}$, and consider also the left trivial binary operations $x *_{0} y=x$, and the right trivial binary operations $x * \sim y=y$ and then to recover $\partial$ as a three term entropic boundary function, for the multi-entropic system $\left(*, *_{0}, *_{\sim}\right)$, by the formula $\partial=\partial^{(*)}-t \partial^{\left(*_{0}\right)}-s \partial^{\left(*_{\sim}\right)}$.

\section{Degeneracy For a WEAK AND VERY WEAK SIMPLICIAL MODULE}

We expand here on Subsections 3.2 and 3.3 and discuss degenerate part of distributive homology in the general context of weak and very weak simplicial modules.

Quandle homology is build in analogy to group homology or Hochschild homology of associate structures. In the unital associative case we deal with simplicial sets (or modules) and it is a classical result of Eilenberg and Mac Lane that the degenerate part of a chain complex is acyclic so homology and normalized homology are isomorphic (see Subsection 3.2). It is not the case for distributive structures, e.g. for quandles or spindles. Quandle homology or even one term distributive homology of spindles may have nontrivial degenerate part. The underlining homological algebra structure is a weak simplicial module and in this case the degenerate part is not necessarily acyclic and the best one can say is that the degenerate part has a natural filtration so yields a spectral sequence which can be used to study degenerate homology. In the concrete case of quandle homology (motivated by and applicable to knot theory) it is proven that the homology (called the rack homology) splits into degenerate and normalized (called the quandle homology) parts [L-N]. Otherwise no clear general connection between degenerate and quandle part were observed. We prove in the joint paper with Krzysztof Putyra $\mathrm{Pr}-\mathrm{Pu}-2$ that the degenerated homology of a quandle is fully determined by quandle homology via a Künneth type formula. 


\section{DEGENERACY FOR A WEAK SIMPLICIAL MODUlE}

Here we give a few general observations about degenerate part of a weak simplicial module. They are related to concrete work in the distributive case done in [Pr-Pu-2].

Consider a weak simplicial module $\left(C_{n}, d_{i}, s_{i}\right)$ (see Subsection 3.2 and Prz-5]). As checked in Corollary 3.4, the filtration by degenerate elements $F_{n}^{p}=\operatorname{span}\left(s_{0}\left(C_{n-1}\right), \ldots, s_{p}\left(C_{n-1}\right)\right)$ is preserved by the boundary operation $\partial_{n}=\sum_{i=0}^{n}(-1)^{i} d_{i}$. In Subsection 3.3 we constructed a degenerate bicomplex $\left(E_{i, j}^{0}, d^{v}, d^{h}\right)$

We discuss here the fact that a weak simplicial complex has also dual filtration (or better to say it has left and write filtrations). We define the dual (or opposite) filtration $\hat{F}_{n}^{p}=\operatorname{span}\left(s_{n-1}\left(C_{n-1}\right), \ldots, s_{n-p}\left(C_{n-1}\right)\right)$.

We start our dual description from a presimplicial module:

If $\left(C_{n} ; d_{i}\right)$ is a presimplicial module then we define $\hat{d}_{i}=d_{n-i}$ and notice that $\left(C_{n} ; \hat{d}_{i}\right)$ is also a presimplicial module with $\hat{\partial}_{n}=(-1)^{n} \partial_{n}$ and unchanged homology. More generally we have:

\section{Proposition 10.1.}

(i) If $\left(C_{n} ; d_{i}\right)$ is a presimplicial module then $\left(C_{n} ; \hat{d}_{i}\right)$ is also a presimplicial module.

(ii) If $s_{i}: C_{n} \rightarrow C_{n+1}, 0 \leq i \leq n$ are degenerate map, define $\hat{s}_{i}=s_{n-i}$. Then if $\left(C_{n} ; d_{i}, s_{i}\right)$ is a (weak, or very weak) simplicial module then $\left(C_{n} ; \hat{d}_{i}, \hat{s}_{i}\right)$ is also a a (weak or very weak) simplicial module.

Proof. (i) For $i<j$ we have $n-j<n-i$, so:

$$
\hat{d}_{i} \hat{d}_{j}=\hat{d}_{i} d_{n-j}=d_{n-1-i} d_{n-j} \stackrel{(1)}{=} d_{n-j} d_{n-i}=\hat{d}_{j-1} \hat{d}_{i} .
$$

(ii) For a better presentation let us list conditions of a simplicial module for $\left(C_{n}, \hat{d}_{i}, \hat{s}_{i}\right)$, one by one:

$$
\begin{gathered}
(\hat{1}) \quad \hat{d}_{i} \hat{d}_{j}=\hat{d}_{j-1} \hat{d}_{i} \text { for } i<j . \\
(\hat{2}) \quad \hat{s}_{i} \hat{s}_{j}=\hat{s}_{j+1} \hat{s}_{i}, \quad 0 \leq i \leq j \leq n, \\
(\hat{3}) \quad \hat{d}_{i} \hat{s}_{j}= \begin{cases}\hat{s}_{j-1} \hat{d}_{i} & \text { if } i<j \\
\hat{s}_{j} \hat{d}_{i-1} & \text { if } i>j+1\end{cases} \\
\left(\hat{4}^{\prime}\right) \quad \hat{d}_{i} \hat{s}_{i}=\hat{d}_{i+1} \hat{s}_{i} . \\
(\hat{4}) \quad \hat{d}_{i} \hat{s}_{i}=\hat{d}_{i+1} \hat{s}_{i}=I d_{M_{n} .} .
\end{gathered}
$$

Proposition 10.1 follows from the following lemma.

Lemma 10.2. Consider $\left(C_{n} ; d_{i}, s_{i}\right)$ and its complementary (dual) $\left(C_{n} ; \hat{d}_{i}, \hat{s}_{i}\right)$, then conditions $(x)$ and $(\hat{x})$ are equivalent 
Proof. Equivalence of (1) and ( $\hat{1})$ was already established. Other parts are equally simple but we prove them for completeness:

$(2) \Leftrightarrow(\hat{2})$ (we assume $i \leq j$ or equivalently $n-j \leq n-i$ ):

$$
\hat{s}_{i} \hat{s}_{j}=\hat{s}_{i} s_{n-j}=s_{n+1-i} s_{n-j} \stackrel{(2)}{=} s_{n-j} s_{n-i}=\hat{s}_{j+1} \hat{s}_{i} .
$$

$(3) \Leftrightarrow(\hat{3})$ First assume that $i<j$ (i.e. $n+1-i>n-j+1)$ then:

$$
\hat{d}_{i} \hat{s}_{j}=d_{n+1-i} s_{n-j} \stackrel{(3)}{=} s_{n-j} d_{n-i}=\hat{s}_{j-1} \hat{d}_{i} .
$$

Second assume that $i>j+1$ (i.e. $n+1-i<n-j$ ), then:

$$
\hat{d}_{i} \hat{s}_{j}=d_{n+1-i} s_{n-j} \stackrel{(3)}{=} s_{n-j-1} d_{n-i+1}=\hat{s}_{j} \hat{d}_{i-1} .
$$

$\left(4^{\prime}\right) \Leftrightarrow\left(\hat{4}^{\prime}\right)$. We have:

$$
\hat{d}_{i} \hat{s}_{i}=d_{n+1-i} s_{n-i} \stackrel{\left(4^{\prime}\right)}{=} d_{n-i} s_{n-i}=\hat{d}_{i+1} \hat{s}_{i} .
$$

$(4) \Leftrightarrow(\hat{4})$. We have:

$$
\hat{d}_{i} \hat{s}_{i}=d_{n+1-i} s_{n-i} \stackrel{(4)}{=} I d
$$

Remark 10.3. If $C_{n}=Z X^{n+1}$ then we can consider the map $\hat{I}$ : $\left(C_{n} ; d_{i}\right) \rightarrow\left(C_{n} ; \hat{d}_{i}\right)$ given by $\hat{I}\left(x_{0}, x_{1}, \ldots, x_{n}\right)=\left(x_{n}, \ldots, x_{1}, x_{0}\right)$ (or succinctly $\hat{I}(\mathbf{x})=\hat{\mathbf{x}})$ ).

Our results ( Proposition 10.1 and Lemma 10.2 hold for very weak simplicial modules, weak simplicial modules, and simplicial modules. In particular, for a weak simplicial module the dual filtration of $C_{n}^{D}$, $\hat{F}_{n}^{p}=\operatorname{span}\left(\hat{s}_{0}\left(C_{n-1}\right), \hat{s}_{1}\left(C_{n-1}\right), \ldots, \hat{s}_{p}\left(C_{n-1}\right)\right.$ leads to a spectral sequence and a bicomplex. Here we give a few general remarks to summarize basic facts:

A weak simplicial module yields two filtrations: $F_{n}^{p}$ and the dual (complementary) one $\hat{F}_{n}^{p}$. By the definition we have

$$
\partial_{n}=\sum_{i=0}^{n}(-1)^{i} d_{i}=(-1)^{n} \sum_{i=0}^{n}(-1)^{i} \hat{d}_{i}=(-1)^{n} \hat{\partial}_{n}
$$

Furthermore, on $s_{p}\left(C_{n-1}\right)$ we have:

$$
\partial_{n} s_{p}=\sum_{i=0}^{n}(-1)^{i} d_{i} s_{p}=\sum_{i=0}^{p-1}(-1)^{i} d_{i} s_{p}+(-1)^{p} d_{p} s_{p}+(-1)^{p+1} d_{p+1} s_{p}+\sum_{i=p+2}^{n}(-1)^{i} d_{i} s_{p} \stackrel{\left(4^{\prime}\right)}{=}
$$




$$
\begin{aligned}
& \sum_{i=0}^{p-1}(-1)^{i} d_{i} s_{p}+\sum_{i=p+2}^{n}(-1)^{i} d_{i} s_{p} \stackrel{(3)}{=} \\
& \sum_{i=0}^{p-1}(-1)^{i} s_{p-1} d_{i}+\sum_{i=p+2}^{n}(-1)^{i} s_{p} d_{i-1} .
\end{aligned}
$$

Clearly $\sum_{i=0}^{p-1}(-1)^{i} s_{p-1} d_{i}\left(C_{n-1}\right)$ belongs to $F_{n}^{p-1}$.

The formulas above lead to the bicomplex with $E_{p, q}^{0}=M_{p, q}=$ $F_{n}^{p} / F_{n}^{p-1}$, where $n=p+q, d^{h}=\sum_{i=0}^{p-1}(-1)^{i} d_{i} s_{p}$ and $d^{v}=\sum_{i=p+2}^{n}(-1)^{i} d_{i} s_{p}$. The equality $d^{h} d^{h}=0=d^{v} d^{v}$ and $d^{h} d^{v}=-d^{v} d^{h}$ follows directly from the weak simplicial module structure.

If we replace the filtration $F_{n}^{p}$ by $\hat{F}_{n}^{p}$ we see that the spectral sequence is modified; it is analogous, but not the same, as when $d^{h}$ is replaced by $d^{v}$.

Remark 10.4. An acute observer will notice immediately that we deal not only with a bicomplex but also with pre-bisimplicial category (set or module). For completeness I recall definitions after [Lod-1], page 459:

We define a bisimplicial object but in a same vain we can define prebisimplicial category, and weak bisimplicial category: "By definition a bisimplicial object in a category $\mathcal{C}$ is a functor

$$
\mathcal{X}: \Delta^{o p} \times \Delta^{o p} \rightarrow \mathcal{C} .
$$

Such a bisimplicial object can be described equivalently by a family of objects $M_{p, q}, p \geq 0, q \geq 0$, together with horizontal and vertical faces and degeneracies:

$$
\begin{aligned}
& d_{i}^{h}: M_{p, q} \rightarrow M_{p-1, q}, \quad s_{i}^{h}: M_{p, q} \rightarrow M_{p+1, q}, \text { where } 0 \leq i \leq p \\
& d_{i}^{v}: M_{p, q} \rightarrow M_{p, q-1}, \quad s_{i}^{v}: M_{p, q} \rightarrow M_{p, q+1}, \text { where } 0 \leq i \leq q
\end{aligned}
$$

which satisfy the classical simplicial relations horizontally and vertically and such that horizontal and vertical operations commute. For any bisimplicial set $X$ there are three (homeomorphic) natural ways to make geometric realization, $|X|$ of $X$. Loday notes that any bisimplicial set $X$ gives rise to the bisimplicial module $R X$ and $H_{*}(|X|, R)=$ $H_{*}(\operatorname{Tot}(R X))$, [Lod-1].

Example 10.5. A natural example of a (pre)-bisimplicial set or module is obtained by a Cartesian (or tensor) product of (pre)-simplicial sets (or modules). Namely:

\footnotetext{
${ }^{27}$ Victoria Lebed studied this before me in context of her prebraided category.
} 
$(\times)$ Let $M_{p, q}=C_{p} \times C_{q}^{\prime}$ where $\left(C_{n}, d_{i}\right)$ and $\left(C_{n}^{\prime}, d_{i}^{\prime}\right)$ are (pre) simplicial sets. We define $d_{i}^{h}=d_{i} \times I d_{C_{q}^{\prime}}$ and $d_{i}^{v}=I d_{C_{p}} \times d_{i}^{\prime}$. In the case $\left(C_{n}, d_{i}, s_{i}\right)$ and $\left(C_{n}^{\prime}, d_{i}^{\prime}, s_{i}^{\prime}\right)$ are (weak)-simplicial sets we get $M_{p, q}$ a (weak)-simplicial set with $s_{i}^{h}=s_{i} \times I d_{C_{q}^{\prime}}$ and $s_{i}^{v}=I d_{C_{p}} \times s_{i}^{\prime}$.

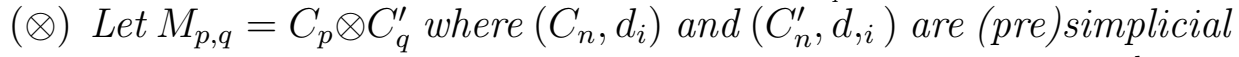
modules. Then $M_{p, q}$ is a (pre)simplicial modules with $d_{i}^{h}=$ $d_{i} \otimes I d_{C_{q}^{\prime}}$ and $d_{i}^{v}=I d_{C_{p}} \otimes d_{i}^{\prime}$. Similarly in the case $\left(C_{n}, d_{i}, s_{i}\right)$ and $\left(C_{n}^{\prime}, d_{i}^{\prime}, s_{i}^{\prime}\right)$ are (weak)-simplicial modules.

\subsection{Right filtration of degenerate distributive elements.}

We restrict ourselves here to a weak simplicial module yielded by a distributive structures.

Let $(X ; *)$ be a spindle that is a magma which is right distributive $((a * b) * c=(a * c) *(b * c))$ and idempotent $(a * a=a)$.

Definition 10.6. (i) Let $\hat{s}_{i}=s_{n-i}: C_{n} \rightarrow C_{n+1}$ is given by $\hat{s}_{i}\left(x_{0}, x_{1}, \ldots, x_{n}\right)=\left(x_{0}, . ., x_{n-i-1}, x_{n-i}, x_{n-i}, x_{n-i+1}, \ldots, x_{n}\right)$,

that is we double the letter on the position $n-i$ (or $i$ from the end) if we count from zero.

(ii) We define $\hat{F}_{n}^{p}=\operatorname{span}\left(\hat{s}_{0}\left(C_{n-1}\right), \hat{s}_{1}\left(C_{n-1}\right), \ldots, \hat{s}_{n-1}\left(C_{n-1}\right)\right)$ in $C_{n}(X)$. $\hat{F}_{n}^{p}$ form a boundary coherent filtration of $C_{n}(X)$ :

$$
0 \subset \hat{F}_{n}^{0} \subset \hat{F}_{n}^{1} \subset \hat{F}_{n}^{n-1}=C_{n}^{D} .
$$

(iii) Let $\hat{G} r_{p}^{n}$ be the associated graded group: $\hat{G} r_{p}^{n}=\hat{F}_{n}^{p} / \hat{F}_{p-1}^{n}$.

If, as before, we define face maps $\hat{d}_{i}=d_{n-i}$ then $\left(C_{n}, \hat{d}_{i}, \hat{s}_{i}\right)$ is a weak simplicial module. Thus $\hat{F}_{n}^{p}$ is a graded filtration ripe for the spectral sequence. (We already noticed that $\hat{\partial}_{n}=\sum_{i=0}^{n}(-1)^{i} \hat{d}_{i}=(-1)^{n} \partial_{n}$.)

We consider the spectral sequence of the filtration starting from the initial page $\hat{E}_{p, q}^{0}=\hat{G} r_{p+q}^{p}$. It is the first main observation of [ $\left.\mathrm{Pr}-\mathrm{Pu}-2\right]$ that the spectral sequence $\left(\hat{E}_{p, q}^{r}, \hat{\partial}_{p, q}^{r}\right)$ stabilizes on the first page, and eventually one term spindle homology can be computed easily from the normalized part.

10.2. Integration maps $\hat{u}_{i}: \hat{F}_{n}^{p} \rightarrow \hat{F}_{n}^{p-1}$.

The main tools to show that the spectral sequence $\hat{E}_{p, q}^{r}$ stabilizes on the first page are the maps (which we can call integration) $\hat{u}_{i}: \hat{F}_{n}^{p} \rightarrow \hat{F}_{n}^{p-1}$, illustrated in Figure 10.1 below 28 , they serve to show that the right

\footnotetext{
${ }^{28}$ In braid notation, $\hat{u}_{i}$ can be expressed as $\sigma_{p} \sigma_{p-1} \ldots \sigma_{i+1} \sigma_{i}$.
} 
degenerated filtration spectral sequence has all $d^{r}(r>0)$ trivial and that homology splits.

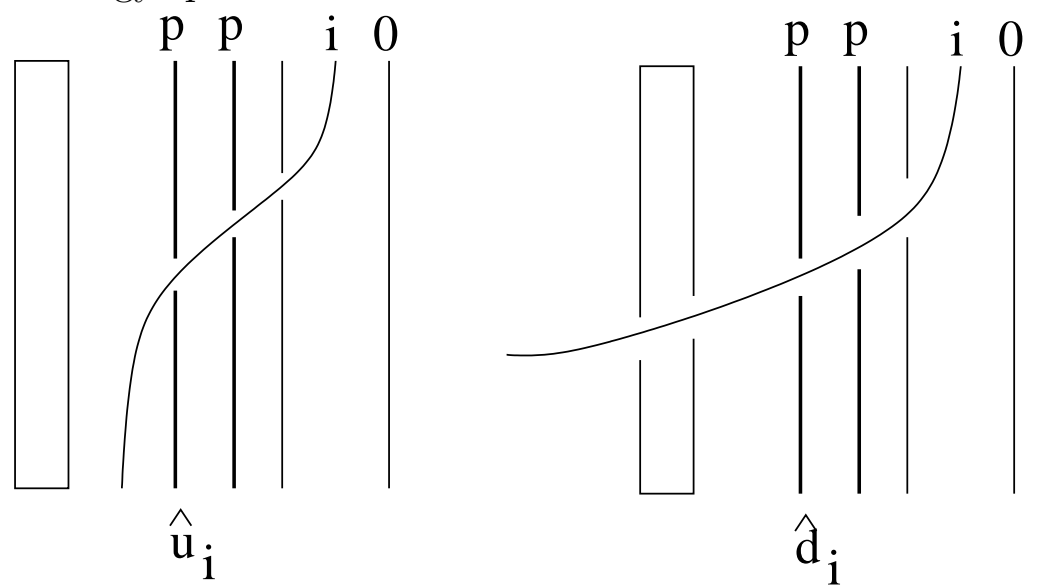

Figure 10.1; The maps $\hat{u}_{i}$ and $\hat{d}_{i}$

We check that the maps $\hat{u}_{i}$ satisfy:

(1) for $i<p$ : $\hat{d}_{i}(y)=\hat{d}_{p+1} \hat{u}_{i}(y)$, where $y \in \hat{s}_{p}\left(C_{n-1}\right)$.

(2) For $p>i_{2}>i_{1}: \hat{d}_{p+1} \hat{u}_{i_{2}-1} \hat{u}_{i_{1}}=\hat{d}_{i_{1}} \hat{u}_{i_{2}}$.

from this follows

(2') For $p>i_{2}>i_{1}: \hat{d}_{p} \hat{u}_{i_{2}-1} \hat{u}_{i_{1}}=\hat{d}_{i_{2}-1} \hat{u}_{i_{1}}$.

(2) and (2') are illustrated in Figure 10.2 and 10.3 below.

(j) For $p>i_{j}>i_{j-1}>\ldots>i_{1} \geq 0$ one has $\hat{d}_{p+1} \hat{u}_{i_{j}-j+1} \ldots \hat{u}_{i_{2}-1} \hat{u}_{i_{1}}=\hat{d}_{i_{1}} \hat{u}_{i_{j}-j+2} \ldots \hat{u}_{i_{3}-1} \hat{u}_{i_{2}}$.

From this follows:

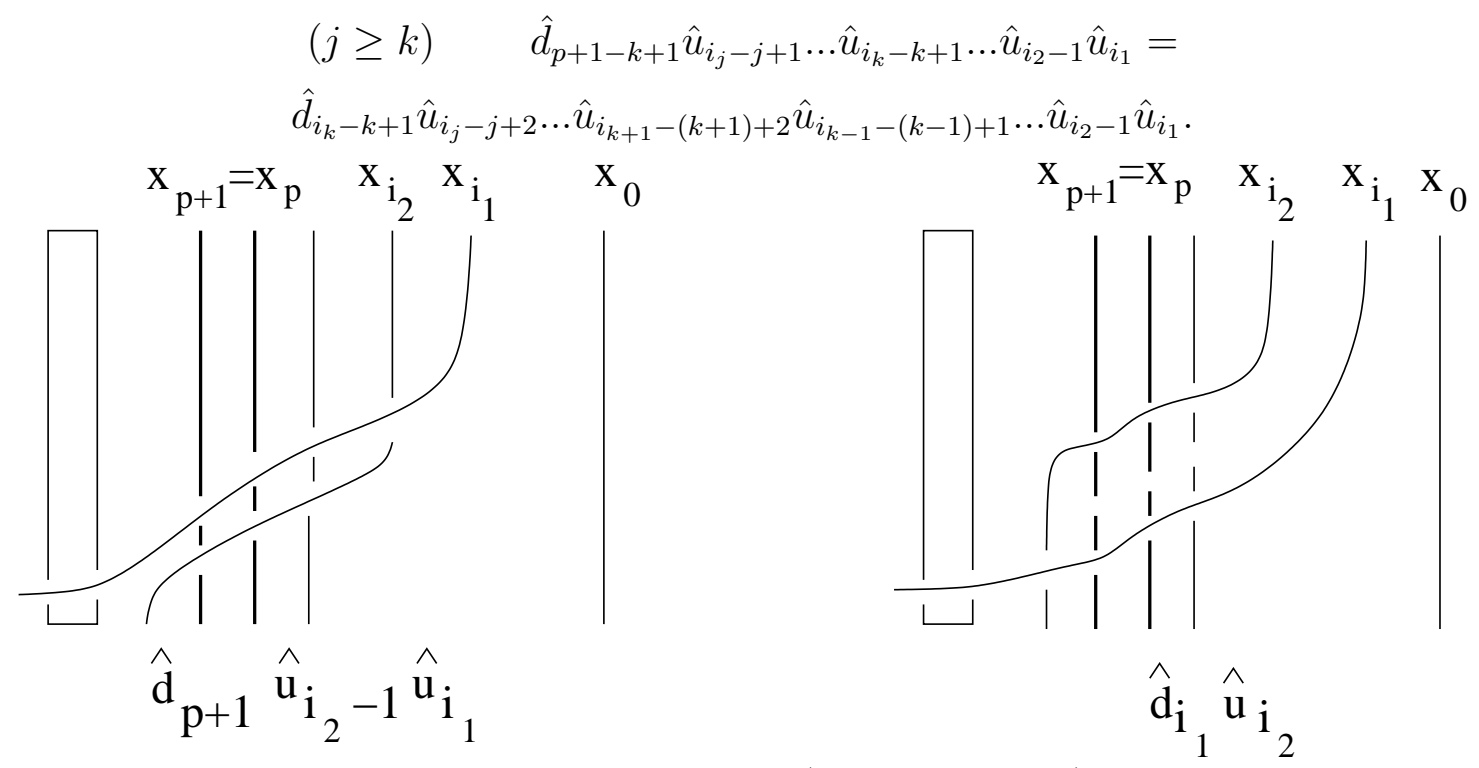

Figure 10.2; Property (2): $\hat{d}_{p+1} \hat{u}_{i_{2}-1} \hat{u}_{i_{1}}=\hat{d}_{i_{1}} \hat{u}_{i_{2}}$ 

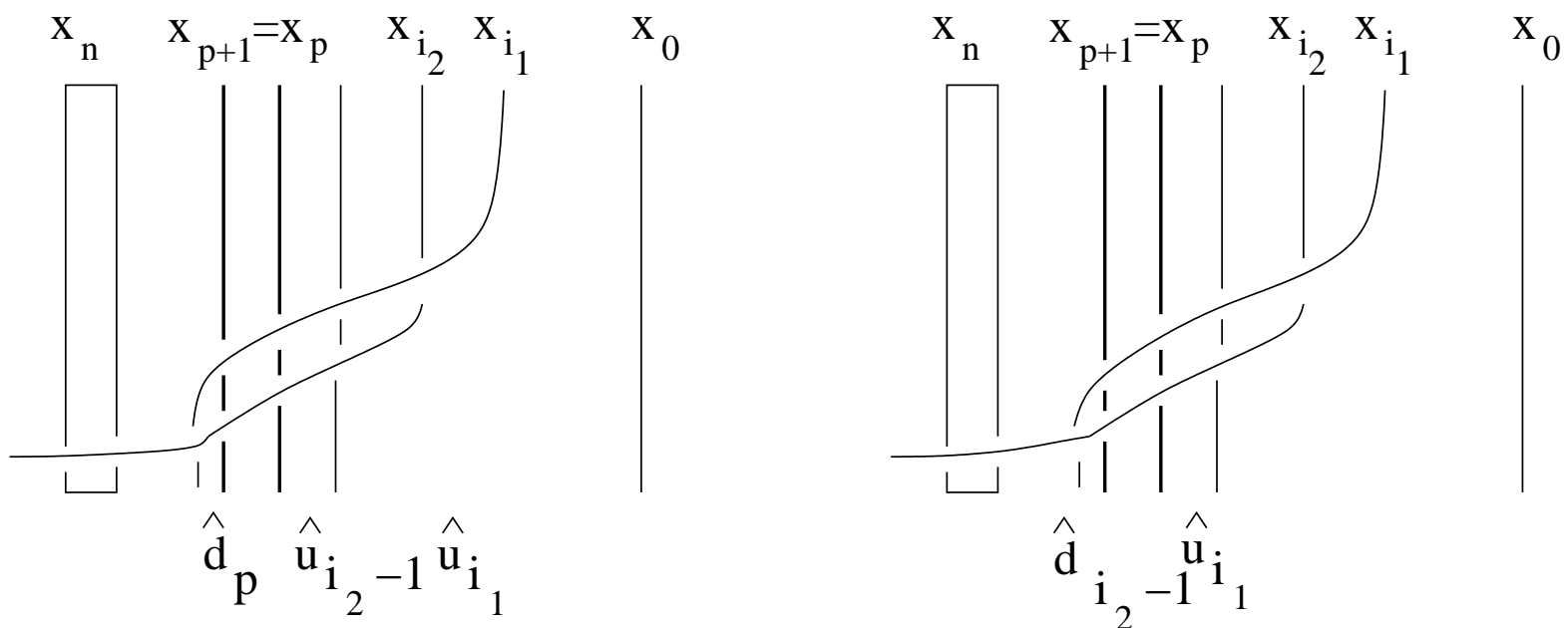

Figure 10.3; Property (2): $\hat{d}_{p} \hat{u}_{i_{2}-1} \hat{u}_{i_{1}}=\hat{d}_{i_{2}-1} \hat{u}_{i_{1}}$

Remark 10.7. We wrote formulas for $\hat{d}_{s} \hat{u}_{i_{k}-k+1} \ldots \hat{u}_{i_{1}-1} \hat{u}_{i_{1}}$ only in the case of $p+1-k \leq s \leq p+1$ as it is needed to compute the degenerate part of one term distributive homology of a spindle.

10.3. Weak simplicial modules with integration. Here we formalize above equations to define weak simplicial module with integration for which spectral sequence stabilizes on $E_{p, q}^{1}$ and homology splits.

We consider a weak simplicial module $\left(C_{n}, d_{i}, s_{i}\right)$ with an additional structure, $u_{i}: s_{p}\left(C_{n-1}\right) \rightarrow s_{p-1}\left(C_{n-1}\right)$ for $i<p$, where maps $u_{i}$ satisfy condition $(\mathrm{j})$ for any $j$. This additional structure allows us to split degenerate part.

Definition 10.8. We say that $\left(C_{n}, d_{i}, s_{i}, u_{i}\right)$ is a weak simplicial module with integration if $\left(C_{n}, d_{i}, s_{i}\right)$ is a weak simplicial module, $u_{i}: F_{n}^{p} \rightarrow$ $F_{n}^{p-1}(0 \leq i<p)$ and the following hold:

(1) $d_{p+1} u_{i}=d_{i}(i<p)$.

(j) $d_{p+1} u_{i_{j}-j+1} \ldots u_{i_{2}-1} u_{i_{1}}=d_{i_{1}} u_{i_{j}-j+2} \ldots u_{i_{3}-1} u_{i_{2}}$, where $p>i_{j}>\ldots>i_{1} \geq 0$.

A weak simplicial module with integration leads to bicomplex which stabilizes on $E_{p, q}^{1}$ and eventually splits using the maps $f_{n}^{p}: F_{n}^{p} / F_{n}^{p-1} \rightarrow$ $\bigoplus_{i=0}^{p} F_{n}^{i} / F_{n}^{i-1}$.

\section{Degeneracy FOR A VERY WEAK SIMPliCIAL MODUle}

We have considered, previously, the degenerate subcomplex in the case of a weak simplicial module, however if $\left(C_{n}, d_{i}, s_{i}\right)$ is only a very 
weak simplicial module, that is $d_{i} s_{i}$ is not necessarily equal to $d_{i+1} s_{i}$, we can still construct the analogue of a degenerate subcomplex (and degenerate filtration (compare Remark. 3.4 in Prz-5]).

Let $\mathcal{C}=\left(C_{n}, d_{i}, s_{i}\right)$ be a very weak simplicial module, that is axioms (1)-(3) of Definition 3.3 hold. We do not necessarily have the condition $d_{i} s_{i}-d_{i+1} s_{i}$ is equal to zero so it is of interest to study an obstruction to zero: $t_{i}=d_{i} s_{i}-d_{i+1} s_{i}$. We have;

Lemma 11.1. Let $t_{i}: C_{n} \rightarrow C_{n}$ where $t_{i}=d_{i} s_{i}-d_{i+1} s_{i}$ in a very weak simplicial module, then:

(i)

$$
d_{i} t_{j}=\left\{\begin{aligned}
t_{j-1} d_{i} & \text { if } i<j \\
0 & \text { if } i=j \\
t_{j} d_{i} & \text { if } i>j
\end{aligned}\right.
$$

(ii) $t_{i} t_{j}=t_{j} t_{i}$.

(iii) It follows from (i) that we have boundary preserving filtrations:

$F_{0}^{t}=t_{0}\left(C_{n}\right) \subset F_{1}^{t}=\operatorname{span}\left(t_{0}\left(C_{n}\right), t_{1}\left(C_{n}\right)\right) \subset \ldots \subset F_{n}^{t}=\operatorname{span}\left(t_{0}\left(C_{n}\right), \ldots, t_{n}\left(C_{n}\right)\right)=F^{t}$

(iii) We have also boundary preserving filtrations:

$$
\begin{gathered}
F_{0}^{t D}=\operatorname{span}\left(t_{0}\left(C_{n}\right), s_{0}\left(C_{n-1}\right) \subset \ldots \subset\right. \\
F_{n-1}^{t D} C_{n}=\operatorname{span}\left(t_{1}\left(C_{n}\right), s_{1}\left(C_{n-1}\right), \ldots, t_{n-1}\left(C_{n}\right), s_{n-1}\left(C_{n-1}\right) \subset\right. \\
F_{n}^{t D} C_{n}=\operatorname{span}\left(t_{1}\left(C_{n}\right), s_{1}\left(C_{n-1}\right), \ldots, t_{n-1}\left(C_{n}\right), s_{n-1}\left(C_{n-1}\right), t_{n}\left(C_{n}\right)\right)=F^{t D} . \\
\text { Or the filtration } \\
0 \subset\left(F_{0}^{D}+F^{t}\right) / F^{t} \subset \ldots \subset\left(F_{n-1}^{D}+F^{t}\right) / F^{t}=F^{t D} / F^{t}
\end{gathered}
$$

(iv) $\left(\partial_{n} t_{p}-t_{p} \partial_{n}\right)\left(t_{p}\left(C_{n}\right)\right) \subset t_{p-1}\left(C_{n}\right)$. In particular, $t_{p}$ is a chain map on $F_{p}^{t}\left(C_{n}^{t}\right) / F_{p-1}^{t}\left(C_{n}^{t}\right)$.

$F^{t D}$ is likely the best proxy of degenerated subchain complex so I call it the generalized degenerated subchain complex of a very weak simplicial module. The quotient $C_{n} / F_{n}^{t D} C_{n}$ is an analogue of a normalized chain complex (in quandle theory the quandle chain complex for any distributive structure, not necessarily spindle or quandle).

Proof. (i) $(i<j$ case $)$ : we have here, $d_{i} t_{j}=d_{i} d_{j} s_{j}-d_{i} d_{j+1} s_{j}=$ $d_{j-1} d_{i} s_{j}-d_{j} d_{i} s_{j}=d_{j-1} s_{j-1} d_{i}-d_{j} s_{j-1} d_{i}=t_{j-1} d_{i}$,

$(i=j$ case $)$ a we have, $: d_{j} t_{j}=d_{j} d_{j} s_{j}-d_{j} d_{j+1} s_{j}=0$,

$\left(i>j\right.$ case): we have, $d_{i} t_{j}=d_{i} d_{j} s_{j}-d_{i} d_{j+1} s_{j}=\mathrm{DO}$

(ii) First we show that:

(ii') $t_{i} s_{j}=s_{j} t_{i}$ for $i<j$ we have, stressing which property is used:

$$
t_{i} s_{j}=\left(d_{i} s_{i}-d_{i+1} s_{i}\right) s_{j}=\left(d_{i}-d_{i+1}\right) s_{i} s_{j} \stackrel{(2) s_{i} s_{j}=s_{j+1} s_{i}}{=}\left(d_{i}-d_{i+1}\right) s_{j+1} s_{i}=
$$




$$
d_{i} s_{j+1} s_{i}-d_{i+1} s_{j+1} s_{i} \stackrel{(3)}{=} s_{j} d_{i} s_{i}-s_{j} d_{i+1} s_{i}=s_{j} t_{i} .
$$

(Similarly we prove that $t_{i} s_{j}=s_{j} t_{i}$ for $i>j$. Now we complete the proof that $t_{i} t_{j}=t_{j} t_{i}$. We have, assuming $i<j$ :

$$
\begin{aligned}
t_{i} t_{j} & =t_{i}\left(d_{j} s_{j}-d_{j+1} s_{j}\right) \stackrel{(i)}{=} d_{j} t_{i} s_{j}-d_{j+1} t_{i} s_{j} \stackrel{\left(i i^{\prime}\right)}{=} \\
& =d_{j} s_{j} t_{i}-d_{j+1} s_{j} t_{i}=t_{j} t_{i} \text { as needed. }
\end{aligned}
$$

(iii) We start from computing $\partial_{n} t_{p}$ stressing each time which property is used:

$$
\begin{gathered}
\partial_{n} t_{p}=\sum_{i=1}^{n}(-1)^{i} d_{i} t_{p}=\sum_{i=0}^{p-1}(-1)^{i} d_{i} t_{p}+0+\sum_{i=p+1}^{n}(-1)^{i} d_{i} t_{p}= \\
\sum_{i=0}^{p-1}(-1)^{i} t_{p-1} d_{i}+\sum_{i=p+1}^{n}(-1)^{i} t_{p} d_{i}
\end{gathered}
$$

This is proving that filtration $F_{p}^{n}$ is boundary preserving and also that we deal with bicomplex. Thus we can construct the spectral sequence from the bicomplex.

Again if we work with a shelf and filtration from the right then it seems to stabilize at $E_{p, q}^{1}$ and homology splits, like in the case of right degenerate filtration of a spindle, $\hat{F}_{n}^{p}$. See Figure 11.1 below for graphical interpretation of $\hat{t}_{i}$. $\hat{u}_{i}^{t}$ will be defined by analogy to $\hat{u}_{i}$.

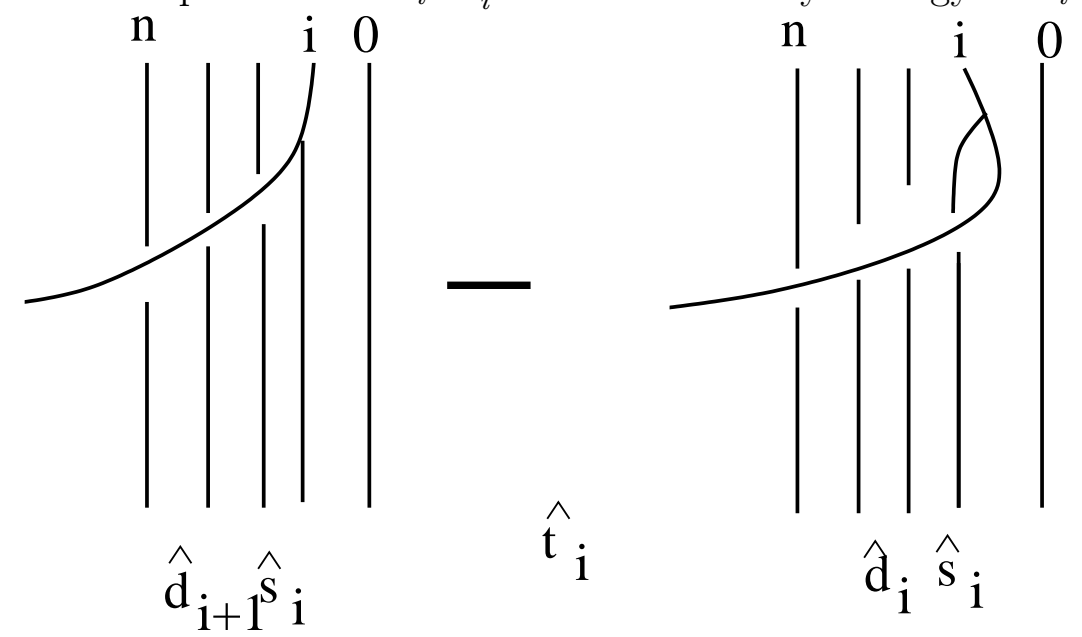

Figure 11.1; The maps $\hat{t}_{i}$ as the difference of $\hat{d}_{i+1} \hat{s}_{i}$ and $\hat{d}_{i} \hat{s}_{i}$

We leave to a reader the development of these ideas, specially in the case of multiterm distributive homology (compare [Pr-Pu-2]). 
11.1. Introduction to t-simplicial objects. We can extract properties of maps $t_{i}$ to obtain a new version of a simplicial object which we will call $t$-simplicial object.

Definition 11.2. Let $\mathcal{C}$ be a category and $\left(X_{n}, d_{i}, t_{i}\right)$ the sequence of objects $X_{n}, n \geq 0$, and morphisms $d_{i}: X_{n} \rightarrow X_{n-1}, t_{i}: X_{n} \rightarrow X_{n}, 0 \leq$ $i \leq n$. We say that $\left(X_{n}, d_{i}, t_{i}\right)$ is a t-simplicial object if the following four conditions hold (the first is the condition of a presimplicial object):

$\left(1^{t}\right) d_{i} d_{j}=d_{j-1} d_{i}$ for $i<j$.

$\left(2^{t}\right) t_{i} t_{j}=t_{j} t_{i}$.

$$
d_{i} t_{j}=\left\{\begin{aligned}
t_{j-1} d_{i} & \text { if } i<j \\
t_{j} d_{i} & \text { if } i>j
\end{aligned}\right.
$$

$\left(4^{t}\right) d_{i} t_{i}=0$.

Let $\left(C_{n}, d_{i}, t_{i}\right)$ be a t-simplicial module, with $\partial_{n}=\sum_{i=0}^{n}(-1)^{i} d_{i}$ then $\left(C_{n}, d_{i}, t_{i}\right)$ leads to a bicomplex $\left(C_{p, q}, d^{h}, d^{v}\right)$ where $d^{h}$ and $d^{v}$ are defined up sign/shift by:

$$
d^{h}=\sum_{i=0}^{p-1}(-1)^{i} d_{i} \text { and } d^{v}=\sum_{i=p+1}^{n}(-1)^{i} d_{i} .
$$

We also can define a $t$ analogue of a bisimplicial object, which we call a $t$-bisimplicial object, $\left(C_{p, q}, d_{i}^{h}, d_{j}^{v}\right), 0 \leq i \leq p, 0 \leq j \leq q$, with $d_{i}^{h}=d_{i}$ and $d_{j}^{v}=d_{p+1+i}$ (however some adjustment is needed to have $p+q=n$ (or just $p+q=n-1)$.

Problem 11.3. The relation $d_{i} t_{i}=0$ is crucial. Can one find for it general setting (say, $t_{i}$ as the marker for horizontal and vertical parts of a bicomplex)? Other applications?

\section{From Distributive homology to YAng-Baxter HOMOLOGY}

We can extend the basic construction from the introduction, still using very naive point of view, as follows: Fix a finite set $X$ and color semi-arcs of $D$ (parts of $D$ from a crossing to a crossing) by elements of $X$ allowing different weights from some ring $k$ for every crossing (following statistical mechanics terminology we call these weights Boltzmann weights). We allow also differentiating between a negative and a positive crossing; see Figure 12.1. 


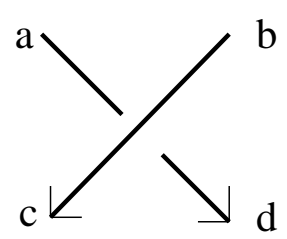

$R_{c d}^{a b}$

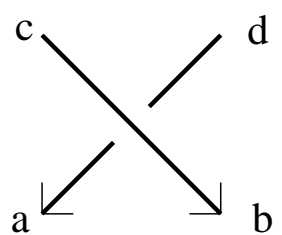

$\overline{\mathrm{R}}_{\mathrm{a} \mathrm{b}}^{\mathrm{c}}$

Figure 12.1; Boltzmann weights $R_{c, d}^{a, b}$ and $\bar{R}_{a, b}^{c, d}$ for positive and negative crossings

We can now generalize the number of colorings to state sum (basic notion of statistical physics) by multiplying Boltzmann weight over all crossings and adding over all colorings:

$$
\operatorname{col}_{(X ; B W)}(X)=\sum_{\phi \in \operatorname{col}_{X}(D)} \prod_{p \in\{\text { crossings }\}} \hat{R}_{c, d}^{a, b}(p)
$$

where $\hat{R}_{c, d}^{a, b}$ is $R_{c, d}^{a, b}$ or $\bar{R}_{c, d}^{a, b}$ depending on whether $p$ is a positive or negative crossing (see [Jones]). Our state sum is an invariant of a diagram but to get a link invariant we should test it on Reidemeister moves. To get analogue of a shelf invariant we start from the third Reidemeister move with all positive crossings. Here we notice that, in analogy to distributivity, where passing through a positive crossing was coded by a map $R: X \times X \rightarrow X \times X$ with $R(a, b)=(b, a * b)$. Thus in the general case passing through a positive crossing is coded by a linear map $R: k X \otimes k X \rightarrow k X \otimes k X$ and in basis $X$ the map $R$ is given by the $|X|^{2} \times|X|^{2}$ matrix with entries $\left(R_{c, d}^{a, b}\right)$. The third Reidemeister move leads to the equality of the following maps $V \otimes V \otimes V \rightarrow V \otimes V \otimes V$ where $V=k X$ :

$$
(R \otimes I d)(I d \otimes R)(R \otimes I d)=(I d \otimes R)(R \otimes I d) I d \otimes R)
$$

This is called the Yang-Baxter equation and $R$ is called a pre-YangBaxter operator. If $R$ is additionally invertible it is called a YangBaxter operator. If entries of $R^{-1}$ are equal to $\bar{R}_{c, d}^{a, b}$ then the state sum is invariant under "parallel" (directly oriented) second Reidemeister move 29

For a given pre-Yang-Baxter operator we attempt to find presimplicial module, from which homology will be derived.

\footnotetext{
${ }^{29}$ We should stress that to find link invariants it suffices to use directly oriented second and third Reidemeister moves in addition to both first Reidemeister moves, as we can restrict ourselves to braids and use the Markov theorem. This point of view was used in Tur.
} 
Figure 12.2 below illustrate various graphical interpretation of the generating morphism $d_{i}$ of the presimplicial category $\Delta_{\text {pre }}^{o p}$. They are related to homology of a set-theoretic Yang-Baxter equation of CarterKamada-Saito [CES-2 and Fenn [FIKM], and to homology of YangBaxter equation of Eisermann [Eis-1, Eis-2]. We should also acknowledge stimulating observations by Ivan Dynnikov.
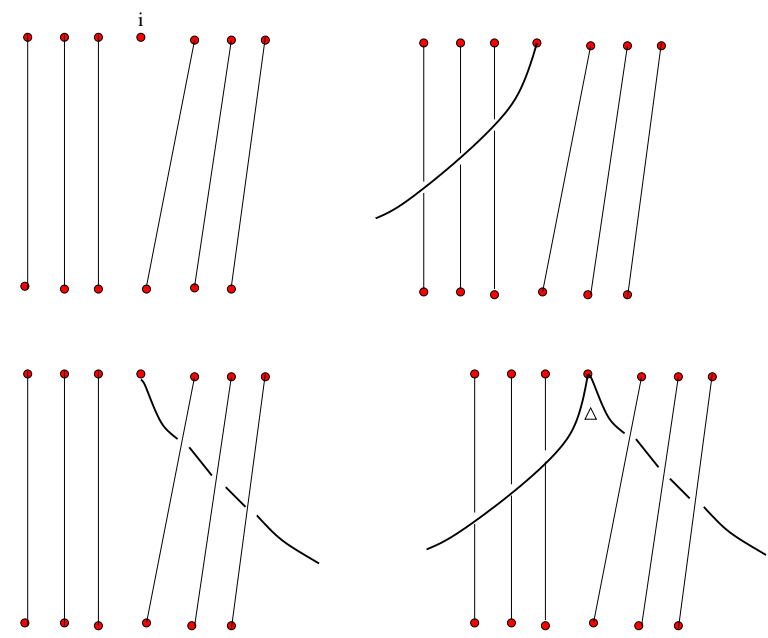

Figure 12.2; Various interpretation of the graphical face map $d_{i}$

12.1. Graphical visualization of Yang-Baxter face maps. The presimplicial set corresponding to (two term) Yang-Baxter homology has the following visualization. In the case of a set-theoretic YangBaxter equation we recover the homology of [CES-2].
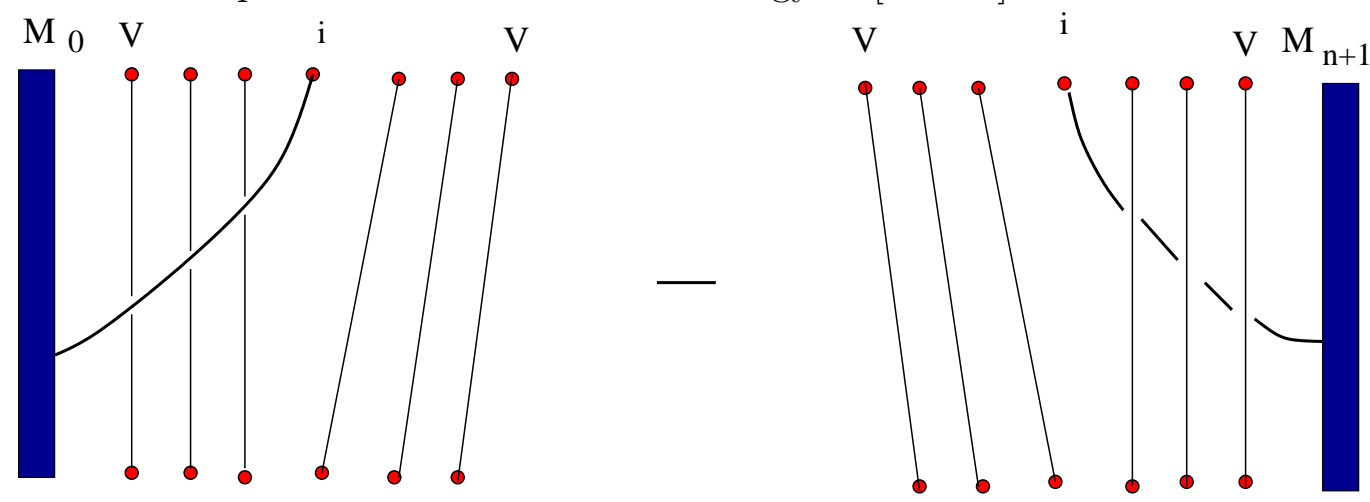

Diagramatic interpretation of a face map d $\underset{i}{\mathrm{Y}-\mathrm{B}}$

Figure 12.3; Graphical interpretation of the face map $d_{i}$ 
Our graphical model allows easy calculation:

Example 12.1. Assume $R: X \times X \rightarrow X \times X$ generates set-theoretic

Yang-Baxter operator with $R(x, y)=\left(R_{1}(x, y), R_{2}(x, y)\right)$. Then

$$
\begin{gathered}
\partial^{Y B}\left(x_{1}, x_{2}, x_{3}, x_{4}\right)=\partial^{\ell}-\partial^{r} \\
\partial^{\ell}\left(x_{1}, x_{2}, x_{3}, x_{4}\right)=\left(\left(x_{2}, x_{3}, x_{4}\right)-\left(R_{2}\left(x_{1}, x_{2}\right), x_{3}, x_{4}\right)+\right. \\
\left(R_{2}\left(x_{1}, R_{1}\left(x_{2}, x_{3}\right), R_{2}\left(x_{2}, x_{3}\right), x_{4}\right)-\left(R _ { 2 } \left(x_{1}, R_{1}\left(x_{2}, R_{1}\left(x_{3}, x_{4}\right), R_{2}\left(x_{2}, R_{1}\left(x_{3}, x_{4}\right), R_{2}\left(x_{3}, x_{4}\right)\right)\right.\right.\right.\right. \\
\partial^{r}\left(x_{1}, x_{2}, x_{3}, x_{4}\right)=\left(R_{1}\left(x_{1}, x_{2}\right), R_{1}\left(R_{2}\left(x_{1}, x_{2}\right), x_{3}\right), R_{1}\left(R_{2}\left(R_{2}\left(x_{1}, x_{2}\right), x_{3}\right), x_{4}\right)-\right. \\
\left(x_{1}, R_{1}\left(x_{2}, x_{3}\right), R_{1}\left(R_{2}\left(x_{2}, x_{3}\right), x_{4}\right)+\left(x_{1}, x_{2}, R_{1}\left(x_{3}, x_{4}\right)\right)-\left(x_{1}, x_{2}, x_{3}\right) .\right.
\end{gathered}
$$

We have generally for any $n$ :

$$
\begin{gathered}
\partial_{n}^{\ell}=\sum_{i=1}^{n}(-1)^{i-1} d_{i}^{\ell} \text { with } \\
d_{i}^{\ell}\left(x_{1}, \ldots, x_{n}\right)= \\
\left.\left(R_{2}\left(x_{1}, R_{1}\left(x_{2}, R_{1}\left(x_{3}, \ldots, R_{1}\left(x_{i-1}, x_{i}\right)\right)\right)\right), \ldots, R_{1}\left(x_{i-1}, x_{i}\right)\right), x_{i+1}, \ldots, x_{n}\right) .
\end{gathered}
$$

Similarly we have, directly from Figure 12.3, for any $n$ :

$$
\begin{gathered}
\partial_{n}^{r}=\sum_{i=1}^{n}(-1)^{i-1} d_{i}^{r} \text { with } \\
d_{i}^{r}\left(x_{1}, \ldots, x_{n}\right)= \\
\left(x_{1}, \ldots, x_{i-1}, R_{1}\left(x_{i}, x_{i+1}\right), \ldots, R_{1}\left(R_{2}\left(R_{2}\left(\ldots\left(R_{2}\left(x_{i}, x_{i+1}\right), x_{i+2}\right), \ldots, x_{n-1}\right) x_{n}\right)\right)\right) .
\end{gathered}
$$

\section{Geometric Realization of Simplicial and CUbiC Sets}

A simplicial (or presimplicial) set (or space, more generally) can be a treated as an instruction of how to glue a topological space from pieces (simplexes in the most natural case). the result is $\mathrm{CW}$ complex or more precisely a $\Delta$-complex in the terminology used in [Hat]. That is an object in $X_{n}$ is a name/label for a an $n$ dimensional simplex, and maps $d_{i}$ (and $s_{i}$ in a weak simplicial case) are giving glueing instruction. Precise description (following [Lod-1]) is given below. The similar construction for a cubic (or pre-cubic) set is described at the end of the section 30. We speculate also what should be a natural generalization of (pre)simplicial and (pre)cubic categories.

\footnotetext{
${ }^{30}$ The definition comes under the general scheme of a co-end, p. 371 of [BHS].
} 
Let $\mathcal{X}$ be a simplicial space (e.g. simplicial set with discrete topology), and $\mathcal{Y}$ a cosimplicial space (e.g. $\mathbf{\Delta}$ ). We define their product over $\Delta$ similar to the tensor product as follows:

$$
\mathcal{X} \times_{\Delta} \mathcal{Y}=\bigcup_{n \geq 0}\left(X_{n} \times Y_{n}\right) / \sim_{r e l}
$$

where $\sim_{r e l}$ is an equivalence relation generated by $\left(x, f_{Y}(y)\right) \sim_{r e l}\left(f_{X}(x), y\right)$, $f:[m] \rightarrow[n], f_{X}$ is the image of $f$ under contravariant functor $\Delta \rightarrow \mathcal{X}$ and $f_{Y}$ is the image of $f$ under the covariant functor $\Delta \rightarrow \mathcal{Y}$.

Definition 13.1. The geometric realization of a simplicial space $\mathcal{X}$ is, by definition, the space

$$
|\mathcal{X}|=\mathcal{X} \times_{\Delta} \boldsymbol{\Delta}=\bigcup_{n \geq 0}\left(X_{n} \times \Delta^{n}\right) / \sim_{r e l}
$$

We restrict our topological spaces in order to have $|\mathcal{X} \times \mathcal{Z}|=|\mathcal{X}| \times|\mathcal{Z}|$.

We can perform our construction also for a presimplicial set (or space). The gluing maps are then limited to that induced by $d_{i}$.

If $\mathcal{X}$ is a simplicial set we may consider only nondegenerate elements in $X_{n}$ (that is elements which are not images under degeneracy maps) and build $|\mathcal{X}|$ as a $C W$ complex: We start from the union of $n$-cells $\Delta^{n}$ indexed by nondegenerate elements in $X_{n}$; the face operations tells us how these cells are glued together to form $|\mathcal{X}|$.

Example 13.2. Let $\mathcal{X}$ be an abstract simplicial complex $X=(V, P)$. If we order its vertices then $\mathcal{X}$ is a presimplicial set with $X_{n}$ being the set of $n$ simplexes of $\mathcal{X}$ and face are defined on each simplex in a standard way $d_{i}\left(x_{0}, \ldots, x_{n}\right)=\left(x_{0}, \ldots, x_{i-1}, x_{i+1}, \ldots, x_{n}\right.$ A copresimplicial space is here the category $\boldsymbol{\Delta}$ with objects $Y_{n}=\Delta^{n}=\left\{\left(y_{0}, \ldots, y_{n}\right) \in\right.$ $\left.\mathbb{R}^{n+1} \mid \sum_{i=0}^{n} y_{i}=1, y_{i} \geq 0\right\}$, one object for any $n \geq 0$. The coface maps $d^{i}: Y_{n} \rightarrow Y_{n+1}$ are given by $d^{i}\left(y_{0}, . ., y_{n}\right)=\left(y_{0}, \ldots, y_{i-1}, 0, y_{i}, \ldots, y_{n}\right)$ (of course $d^{j} d^{i}=d^{i} d^{j-1}$ for $\left.i<j\right)$. Then the topological realization $|\mathcal{X}|$ is a standard geometric simplicial complex associated to $\mathcal{X}$, that is

$$
|X|=\bigcup_{n \geq 0}\left(X_{n} \times \Delta^{n}\right) /\left(x, d^{i}(y)=\left(d_{i}(x), y\right) \text { for } x \in X_{n} \text { and } y \in \Delta^{n-1},\right.
$$

$X_{n}$ with discrete topology and $|X|$ with quotient topology.

13.1. Geometric realization of a (pre)cubic set. First we define a precubic category $\square_{\text {pre }}$.

Definition 13.3. the precubic category $\square_{\text {pre }}$ has as objects non-negative integers $[n]$ interpreted as $n$ points ([0] has the empty object), see Figure 13.1. Thus the objects are the same as in presimplicial $\Delta_{\text {pre }}$ category except the grading shift (now $[n]$ is in grading $n$ ). 


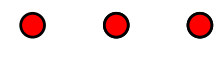

[3]
[4])

Figure 13.1; interpreting objects [1], [2], [3] and [4] in $\square_{\text {pre }}$ category

Morphisms are strictly increasing maps that is $f \in \operatorname{Mor}([m],[n])$ if $f:(1,2, \ldots, m) \rightarrow(1,2, \ldots, n)$ and $i$ j implies $f(i)<f(j)$ with an additional data that points which are not in the image of $f$ have 0 or 1 associated to them. Morphisms are generated by maps $d_{\epsilon}^{i}(1,2, \ldots, n)=$ $(1, \ldots, i-1, i+1, \ldots, n)$ and the point $i$ has marker $\epsilon$ (on the picture marker 0 is denoted by $\leftarrow$ ) (here $1 \leq i \leq n+1, \epsilon=0$ or 1 ); see Figure 13.2 .

The presimplicial category $\Delta_{\text {pre }}$ is the quotient of the pre-cubic category $\square_{\text {pre }}$, but this functor is not that interesting in applications. The proper functor is related with triangulation of a cube [Cla].

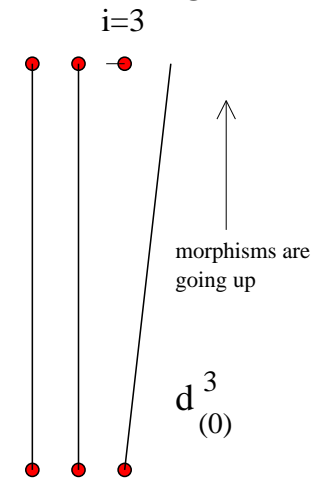

Figure 13.2; morphism (co-face map) $d_{(0)}^{3}$ from [3] to [4]

Definition 13.4. (i) A pre-cubic category is a contravariant functor from a pre-cubic category $\square_{\text {pre }}$ to a given category, $\mathcal{C}$, or, equivalently, a covariant functor $\mathcal{F}: \square_{\text {pre }}^{o p} \rightarrow \mathcal{C}$. We can also say that a pre-cubic category is a sequence of objects $X_{n}$ in $O b(\mathcal{C})$, and morphisms $d_{i}^{\epsilon}: X_{n} \rightarrow X_{n-1}$ satisfying $d_{i}^{\alpha} d_{j}^{\beta}=d_{j-1}^{\beta} d_{i}^{\alpha}$ for $i<j$. The category $\square_{\text {pre }}^{o p}$ is visualized by the same diagrams as the category $\square_{\text {pre }}$ except morphisms will be read from the top to the bottom. 
(ii) A co-pre-cubic category is a covariant functor from a pre-cubic category $\square_{\text {pre }}$ to a given category, $\mathcal{C}$. A basic example of a copre-cubic space $(\mathcal{C}=T O P)$ is given by choosing $X^{n}=I^{n}=$ $\left\{\left(x_{1}, \ldots, x_{n}\right) \in \mathbb{R}^{n} \mid 0 \leq x_{i} \leq 1\right\}$ and morphisms: $d_{\epsilon}^{i}\left(x_{1}, . ., x_{n}\right)=$ $\left(x_{1}, \ldots, x_{i-1}, \epsilon, x_{i+1}, \ldots, x_{n}\right)$. Let us denote this co-pre-cubic space by $\boldsymbol{\mathbf { a }}_{\text {pre }}$.

The basic co-pre-cubic space, $\mathbf{\square}_{\text {pre }}$, can be enriched by degenerate (projection) morphisms $s^{i}:[n] \rightarrow[n-1]$ for $1 \leq i \leq n$, given by $s^{i}\left(x_{1}, \ldots, x_{n}\right)=\left(x_{1}, \ldots, x_{i-1}, x_{i+1}, \ldots, x_{n}\right)$. We denote the new category by $\square$. Then the morphisms $d_{\epsilon}^{i}$ and $s^{i}$ (with the domain $X_{n}=I^{n}$ ) satisfy:

(1) $d_{\beta}^{j} d_{\alpha}^{i}=d_{\alpha}^{i} d_{\beta}^{j-1}$ for $i<j$,

(2) $s^{j} s^{i}=s^{i} s^{j+1}$ for $i \leq j$,

(3) $s^{j} d_{\epsilon}^{i}= \begin{cases}d_{\epsilon}^{i} s^{j-1} & \text { if } i<j \\ d_{\epsilon}^{i-1} s^{j} & \text { if } i>j\end{cases}$

(4) $s^{i} d_{\epsilon}^{i}=I d_{I^{n}}$.

The axioms of the cubic category are modeled on axioms (1)-(4) more precisely:

Definition 13.5. (i) The cubic category $\square$ is composed of objects $[n]$, face maps $d_{\epsilon}^{i}$ (as in pre-cubic category $\square_{\text {pre }}$ ) and degeneracy maps $s_{i}:[n-1] \rightarrow[n] \quad(1 \leq i \leq n$ and relations between morphisms in the category are given by axioms (1)-(4).

(ii) The cubic category is a functor $\mathcal{F}: \square^{o p} \rightarrow \mathcal{C}$. The classical example (of a cubic space) is giving by an approach to singular cubic homology. Here, for a topological space $T, \mathcal{F}([n])$ is the set of all continuous maps $f: I^{n} \rightarrow T . d_{i}^{\epsilon}: \mathcal{F}([n]) \rightarrow \mathcal{F}([n-1])$ is given by $d_{i}^{\epsilon}(f)=f d_{\epsilon}^{i}: I^{n-1} \stackrel{d_{\epsilon}^{i}}{\rightarrow} I^{n} \stackrel{f}{\rightarrow} T$.

(iii) The co-cubic category is a functor $\mathcal{F}: \square \rightarrow \mathcal{C}$. The classical example $\left(I^{n}, d_{\epsilon}^{i}, s^{i}\right)$ was described before as a motivation for a cubic category, $\mathbf{\square}$. This example is used as a building block of a geometric realization of a pre-cubic and cubic set (Definitions 13.6. 13.8).

As we observed already, the pre-cubic category leads to two presimplicial categories $\left(X_{n}, d_{i}^{(0)}\right)$ and $\left(X_{n}, d_{i}^{1)}\right.$ (with shifted grading). Conversely, if we have two presimplicial categories so that $d_{i}^{\alpha} d_{j}^{\beta}=d_{j-1}^{\beta} d_{i}^{\alpha}$ then we combine them to in pre-cubic category (with grade shift).

With degenerate maps situation is not that clear as a condition (3)(4) of a simplicial category only partially agree with the analogous conditions of a cubic category. 
The geometrical realization of a cubical and precubical set (or space) is analogous to that for simplicial or presimplicial sets (space). We write below a formal definition only in the case of a pre-cubic set (space) as degeneracies of a cubic set are not necessarily the one used in knot theory.

Definition 13.6. The geometric realization of a precubic set is a $C W$ complex defined as follows (notice that $X_{n}$ is indexing cubes and precubic structure gives an instruction how to glue the cubes together):

$$
|\mathcal{X}|=\mathcal{X} \times_{\square_{p r e}} \mathbf{\square}=\bigcup_{n \geq 0}\left(X_{n} \times I^{n}\right) / \sim_{r e l}
$$

where $\sim_{\text {rel }}$ is an equivalence relation generated by $\left(x, d_{\epsilon}^{i}(y)\right) \sim_{\text {rel }}\left(d_{i}^{\epsilon}(x), y\right)$, and, as before $d_{\epsilon}^{i}: I^{n-1} \rightarrow I^{n}$ and $d_{i}^{\epsilon}: X_{i} \rightarrow X_{i-1}, x \in X_{n}$, and $y \in I^{n-1}$.

More generally:

Definition 13.7. Let $\mathcal{X}$ be a pre-cubic space (e.g. pre-cubic set with discrete topology), and $\mathcal{Y}$ a co-pre-cubic space (e.g. $\left.\mathbf{\square}_{\text {pre }}\right)$. The we define

$$
\mathcal{X} \times_{\square_{p r e}} \mathcal{Y}=\bigcup_{n \geq 0}\left(X_{n} \times Y_{n}\right) / \sim_{r e l}
$$

where $\sim_{\text {rel }}$ is an equivalence relation generated by $\left(x, d_{\epsilon}^{i}(y)\right) \sim_{\text {rel }}\left(d_{i}^{\epsilon}(x), y\right)$.

If $\left(X_{n}, d_{i}^{\epsilon}, s_{i}\right)$ is a cubic set (or space) we define its geometric realization similarly to Definitions 13.6] and 13.7, but including also $s_{i}, s^{i}$ as gluing morphisms (effectively dividing by degenerate part, which is not necessary acyclic).

Definition 13.8. Let $\mathcal{X}$ be a cubic set and $\mathcal{Y}$ the co-cubic space $\left(I^{n}, d_{\epsilon}^{i}, s^{i}\right)$ then we define the geometric realization $|\mathcal{X}|$ of $\mathcal{X}$ as

$$
|\mathcal{X}|=\mathcal{X} \times_{\square} \mathbf{\square}=\bigcup_{n \geq 0}\left(X_{n} \times I^{n}\right) / \sim_{r e l}
$$

where $\sim_{\text {rel }}$ is an equivalence relation generated by $\left(x, d_{\epsilon}^{i}(y)\right) \sim_{r e l}\left(d_{i}^{\epsilon}(x), y\right)$, and analogously with $s_{i}$ and $s^{i}$.

\section{Higher DiMENSIONAL KNOT THEORY $M^{n} \rightarrow \mathbb{R}^{n+2}$}

Many ideas described in this paper can be applied to higher dimensional knot theory, were we study embeddings of $n$ dimensional (mostly orientable) manifolds in $\mathbb{R}^{n+2}$. For this we direct readers to CKS and [Pr-Ro-2]. 


\section{ACKNOWLEDGEMENTS}

J. H. Przytycki was partially supported by the NSA-AMS 091111 grant, by the GWU-REF grant, and Simons Collaboration Grant316446 .

\section{REFERENCES}

[A-G] M. Andruskiewitsch, M. Grana, From racks to pointed Hopf algebras, Adv. in Math., 178 (2), 2002, 177-243;

e-print: http://front.math.ucdavis.edu/0202.5084

[B-T] F. R. Beyl, J. Tappe, Group Extensions, Representations, and the Schur Multiplicator (Lecture Notes in Mathematics), Springer 1982.

[Blo-1] A. Bloh, On a generalization of the concept of Lie algebra, Dokl. Akad. Nauk SSSR 165, 1965, 471473.

[Blo-2] A. Bloh, Cartan-Eilenberg homology theory for a generalized class of Lie algebras, Dokl. Akad. Nauk SSSR, 175(8), 1967, 824826.

[Bou] N. Bourbaki, Elements of mathematics, Algebra (Algebraic Structures),

Hermann, Paris; Addison-Wesley Publishing Co., Reading Mass., 1974, xxiii+709 pp.

[Bro] K. S. Brown, Cohomology of groups, Graduate Texts in Mathematics, 87, Springer-Verlag, 1982.

[BHS] R. Brown, P. J. Higgins, R. Silvera, Nonabelian Algebraic Topology, Tracts in Mathematics 15, EMS, 2011.

[Bru] R. H. Bruck, A survey of binary systems, Springer, Berlin 1958.

[Bur-1] Celestin Burstin, Belorusskij "Memorial" hhtp://nekropole.info/en/Celestin-Burstin, accessed November 29, 2013.

[Bur-2] C. Burstin, Letter to A.Einstein, Minsk 1933.

[Bur-M] Burstin's Matura, Nowa Reforma (afternoon edition), 285 Krakow, Monday, July 24, 1907. (Tarnopol, 22 czerwca. Egzamin dojrzałości w gimnazyum I w Tarnopolu, ...Burstin Celestyn (ekst.))

[B-M] C. Burstin and W. Mayer, Distributive Gruppen von endlicher Ordnung, $J$. Reine Angew. Math., vol. 160, 1929, pp. 111-130;

Translation "Finite Distributive Groups" by Ansgar Wenzel is available at e-print: arXiv:1403.6326 [math.GR]

[Ca-E] H. Cartan, S. Eilenberg, Homological Algebra, Princeton University Press, 1956.

[Car] J. S. Carter, A Survey of Quandle Ideas, the chapter in the book Introductory Lectures on Knot Theory: Selected Lectures presented at the Advanced School and Conference on Knot Theory and its Applications to Physics and Biology, ICTP, Trieste, Italy, 11 - 29 May 2009, World Scientific, Series on Knots and Everything - Vol. 46, 2012, 22-53; e-print: http://arxiv.org/abs/1002.4429

[CES-1] J. S. Carter, M. Elhamdadi, M. Saito, Twisted quandle homology theory and cocycle knot invariants, Algebraic 83 Geometric Topology, Volume 2, 2002, 95-135.

[CES-2] J. S. Carter, M. Elhamdadi, M. Saito, Homology Theory for the SetTheoretic Yang-Baxter Equation and Knot Invariants from Generalizations 
of Quandles, Fund. Math. 184, 2004, 31-54;

e-print: http://front.math.ucdavis.edu/0206.5255

[CJKS] S. Carter, D. Jelsovsky, S. Kamada, M. Saito, Quandle homology groups, their Betti numbers, and virtual knots, J. Pure Appl. Algebra 157, 2001, 135155; e-print: http://front.math.ucdavis.edu/math.GT/9909161

[CKS] S. Carter, S. Kamada, M. Saito, Surfaces in 4-space, Encyclopaedia of Mathematical Sciences, Low-Dimensional Topology III, R.V.Gamkrelidze, V.A.Vassiliev, Eds., Springer-Verlag, 2004, 213pp.

[Ch-E] C. Chevalley, S. Eilenberg, Cohomology theory of Lie groups and Lie algebras, Trans. Amer. Math. Soc., 63, 1948, 85-124.

[Cla] F. J. B. J. Clauwens, The algebra of rack and quandle cohomology, Journal of Knot Theory and Its Ramifications, 20(11), 2011, 1487-1535; e-print: http://front.math.ucdavis . edu/1004.4423

[C-W] J. H. Conway, G. Wraith, Correspondence 1959.

[Cr] A. S. Crans, Lie 2-algebras, Ph.D. Dissertation, 2004, UC Riverside, available at arXiv:math.QA/0409602.

[C-N] A. Crans, S. Nelson, Hom Quandles; Journal of Knot Theory and Its Ramifications, 23(2), 2014, 1450010 1-18; e-print: http://arxiv.org/abs/1310.0852

[CPP] A. Crans, J. H. Przytycki, K. Putyra, Torsion in one term distributive homology, Fundamenta Mathematicae, 225, 2014, 75-94; e-print: arXiv:1306.1506 [math.GT]

[Cro] R. H. Crowell, Knots and Wheels, National Council of Teachers of Mathematics (N.C.T.M.) Yearbook, 1961.

[C-F] R. H. Crowell, R. H. Fox, An introduction to knot theory, Ginn and Co., 1963, 10+182 pp. (second edition Springer-Verlag, New York, 1977, third edition Dover publications, Inc. 2008).

[Cuv] Christian Cuvier, Homologie de Leibniz et homologie de Hochschild, C. $R$. Acad. Sci. Paris Sr. I Math., 313(9), 1991, 569572. (Christian Cuvier was a student of Loday, who gave up mathematics soon after his thesis; he was the first to publish a detailed study of Leibniz algebras and their homologies, in his thesis and in the above paper; but compare Blo-1, Blo-2]

[Da-Ki] J. F. Davis, P. Kirk, Lectures Notes in Algebraic Topology,Graduate Studies in Mathematics, Vol. 35, American Mathematical Society, Providence, Rhode Island, 367+xv pages, 2001.

[DPT] M. K. Dą̧bkowski, J. H. Przytycki, A. A. Togha, Non-left-orderable 3manifold groups, Canadian Mathematical Bulletin, 48(1), 2005, 32-40. e-print: http://front.math.ucdavis.edu/math.GT/0302098

[Dehe] R. Deheuvels, Homologies des ensembles ordonnés et des espaces topologiques, Bull. Soc. Math. de France, 90, 261-321, 1962.

[Deh-1] P. Dehornoy, Multiple left distributive systems, Comm. Math. Univ. Carol. 38,4 (1997) 615625.

[Deh-2] P. Dehornoy, Braids and self-distributivity, Progress in Mathematics Vol. 192, Birkhauser Verlag, Basel - Boston - Berlin, 2000.

[Deh-3] P. Dehornoy, Laver's results and low-dimensional topology, For Lavers's memorial book, to appear in Archive for Math Logic.

[E-M-1] S. Eilenberg, S. Mac Lane, Cohomology theory in abstract groups. I, Ann. of Math., 48, 51-78, 1947. 
[E-M-2] S. Eilenberg, S. Mac Lane, Acyclic models, Am. J. Math,, 75, 1953, 189199.

[E-Z] S. Eilenberg, J. Zilber, Semi-simplicial complexes and singular homology, Ann. of Math. (2) 51, 1950, 499-513.

[Eis-1] M. Eisermann, Yang-Baxter deformations of quandles and racks, Algebr. Geom. Topol. 5, 2005, 537-562; e-print: http://front.math.ucdavis.edu/0409.5202

[Eis-2] M. Eisermann, Yang-Baxter deformations and rack cohomology, Trans. Amer. Math. Soc., in press, 2014; e-print: http://front.math.ucdavis .edu/0808.0108

[Fenn] R. Fenn, Tackling the Trefoils, Journal of Knot Theory and its Ramifications, 21(13), 2012, 1240004; e-print: arXiv:1110.0582v1; also available at http://www.maths.sussex.ac.uk/Staff/RAF/Maths/

[FIKM] R. Fenn, D. P. Ilyutko, L. H. Kauffman, V. Manturow, Unsolved Problems in Virtual Knot Theory and Combinatorial Knot Theory, Banach Center Publications, 103, 2014, to appear; e-print: arXiv:1409.2823 [math.GT]

[F-R] R. Fenn, C. Rourke, Racks and links in codimension two, Journal of Knot Theory and its Ramifications 1(4), 1992, 343-406; e-print: http://www.maths.sussex.ac.uk/Staff/RAF/Maths/racks.ps

[FRS-1] R. Fenn, C. Rourke, B. Sanderson, An introduction to species and the rack space, in M.E.Bozhuyuk (ed), Topics in Knot Theory (Proceedings of the Topology Conference, Erzurum), NATO Adv. Sci. Inst. Ser. C. Math. Phys. Sci., 399, Kluver Academic Publishers, 33-35, 1993.

[FRS-2] R. Fenn, C. Rourke, B. Sanderson, Trunks and classifying spaces, Applied Categorical Structures, 3, 1995, 321-356.

[FRS-3] R. Fenn, C. Rourke, B. J. Sanderson, James bundles and applications, preprint 1995 http://www.maths.warwick.ac.uk/ cpr/ftp/james.ps Published in Proc. London Math. Soc. (3) 89, no. 1, 2004, 217-240.

[FRS-4] R. Fenn, C. Rourke, B. J. Sanderson, The rack space, Trans. Amer. Math. Soc., 2007.

[Fra] A. Frabetti, Dialgebra (co)homology with coefficients, in J.-L.Loday, A.Frabetti,F.Chapoton, F.Goichot, Dialgebras and Related Operads, Lectures Notes in Mathematics, 1763, Springer-Verlag, 2001, pp. 67-103.

[Fra1] A. Frabetti, Dialgebra homology of associative algebras, C.R. Acad. Sci. Paris, 325, 1997, 135-140.

[Gr] M. Greene, Some results in geometric topology and geometry, Ph.D. thesis, University of Warwick, advisor: Brian Sanderson, 1997.

[Hat] A. Hatcher, Algebraic Topology, Cambridge University Press, 2002. http://www.math.cornell.edu/ hatcher/AT/ATch3.pdf

[Hoch] G. Hochschild, On the cohomology groups of an associative algebra, Annals of Math., 46, 1945, 58-67.

[Hop] H. Hopf, Fundamentalgruppe und zweite Bettische Gruppe, Comment. Math. Helv., 14, 1942, 257-309.

[Hur] W. Hurewicz, Beiträge, zur Topologie der deformationen. IV Asphärische Räume, Nederl. Akad. Wetensch. Proc., 39, 1936, 215-224.

[Isa] W. Isaacson, Einstein: His Life and Universe, Simon and Shuster, 2007. 
[IIJO] A. Ishii, M. Iwakiri, Y. Jang, K.Oshiro, A G-family of quandles and handlebody-knots, preprint, April 2012;

e-print: http://front.math.ucdavis.edu/1205.1855

[Jones] V. F. R. Jones, On knot invariants related to some statistical mechanical models, Pacific J. Math., 137 (1989), no. 2, 311-334.

[Joy-1] D. Joyce, PhD thesis 1979 (adviser D.Freyd)

[Joy-2] D. Joyce, A classifying invariant of knots: the knot quandle, J. Pure Appl. Algebra 23, 1982, 37-65;

[Lar] D. Larue, Left-distributive idempotent algebras, Comm. in Algebra, 27-5, 1999, 2003-2029.

[Leb-1] V. Lebed, Braided objects: unifying algebraic structures and categorifying virtual braids December 2012, Thesis (Ph.D.), Université Paris 7;

[Leb-2] V. Lebed, Homologies of algebraic structures via braidings and quantum shuffles, J. Algebra, 391, 2013, 152192.

[L-N] R. A. Litherland and S. Nelson, The Betti numbers of some finite racks, $J$. Pure Appl. Algebra, 178, 2003, 187-202.

[Lod-1] J-L Loday, Cyclic Homology, Grund. Math. Wissen. Band 301, SpringerVerlag, Berlin, 1992 (second edition, 1998).

[Lod-2] J-L Loday, Une version non commutative des algbres de Lie: les algbres de Leibniz, 1993 Enseign. Math., (2) 39 (34), 1993, 269293.

[L-T] J-L Loday, Pirashvili Teimuraz, Universal enveloping algebras of Leibniz algebras and (co)homology, Mathematische Annalen, 296(1), 1993, 139158.

[Mac] S. Mac Lane, A Mathematical Autobiography, by Saunders Mac Lane, AK Peters, 2005. Hardcover, 358 pp.

[Ma-Bi] S. Mac Lane, G. Birkhoff, Algebra, Macmillan, New York (1967). Third edition, AMS Chelsea Publishing.

[Mal] L. Maligranda, Antoni Lomnicki (1881 - 1941), Wiadomosci Matematyczne, XLIV, 2008, 61-112.

[May] J. P. May, Simplicial objects in algebraic topology, The University of Chicago Press, 1967.

[Mez] G. T. Mezera, Embedding Groups Into Distributive Subsets of the Monoid of Binary Operations, Involve, accepted for publication; e-print: http://front.math.ucdavis.edu/1211.0031

[Mil] J. Milnor, The geometric realization of a semi-simplicial complex, Ann. of Math., 65, 1957, 357-362.

[Mio] J. Mioduszewski, Celestyn Burstin (1888-1938) - członek Polskiego Towarzystwa Matematycznego, in: Matematycy polskiego pochodzenia na obczyznie, XI Szkoła Historii Matematyki (Kołobrzeg, May 1997), red. S.Fudali, Wydawnictwo Naukowe Uniwersytetu Szczecińskiego, Szczecin 1998, 161-165.

[Mov] Yu. M. Movsisyan, The multiplicative group of field and hyperidentities, Izv. Akad. Nauk SSSR Ser. Mat., 53 (1989), 1040-1055. English transl. in Math. USSR Izvestiya, 35 (1990) 337-391)

[Nieb] M. Niebrzydowski, Knots, categories, and dynamics, presentation at Knots in Washington XXXV in December of 2012; http://at.yorku.ca/cgi-bin/abstract/cbfw-04 
[N-P-1] M. Niebrzydowski, J. H. Przytycki, Burnside Kei, Fundamenta Mathematicae 190, June, 2006, 211-229;

e-print: http://front.math.ucdavis.edu/math.GT/0601004

[N-P-2] M. Niebrzydowski, J. H. Przytycki, Homology of dihedral quandles, J. Pure Appl. Algebra, 213, 2009, 742-755;

e-print: http://front.math.ucdavis.edu/math.GT/0611803

[N-P-3] M. Niebrzydowski, J. H. Przytycki, Homology operations on homology of quandles, Journal of Algebra, 324, 2010, pp. 1529-1548; e-print: http://front.math.ucdavis.edu/0907.4732

[N-P-4] M. Niebrzydowski, J. H. Przytycki, Entropic magmas, their homology, and related invariants of links and graphs, Algebraic $\& 3$ Geometric Topology (AGT), 13(6), 2013, 3223-3243; e-print: arXiv:1211.2951 [math.GT]

[Nos] T. Nosaka, On quandle homology groups of Alexander quandles of prime order, Trans. Amer. Math. Soc., 365, 2013, 3413-3436.

[Pei] Benjamin Peirce, Linear Associative Algebra, Read before the National Academy of Sciences, Washington City, 1870; http://www.math.harvard.edu/history/peirce_algebra/index.html

[Peir] C. S. Peirce, On the algebra of logic, American J. of Math., 3(1), 1880, 15-57.

[Prz-1] J. H. Przytycki, Survey on recent invariants in classical knot theory, Warsaw University, Preprints 6,8,9; Warszawa, 1986;

e-print: http://front.math.ucdavis.edu/0810.4191

[Prz-2] J. H. Przytycki, Teoria węztów: podejście kombinatoryczne, (Knots: combinatorial approach to the knot theory), Script, Warsaw, August 1995, 240+XLVIIIpp.

[Prz-3] J. H. Przytycki, 3-coloring and other elementary invariants of knots, $B a$ nach Center Publications, Vol. 42, Knot Theory, 1998, 275-295; e-print: http://arxiv.org/abs/math.GT/0608172

[Prz-4] J. H. Przytycki, When the theories meet: Khovanov homology as Hochschild homology of links, Quantum Topology, 1(2), 2010, 93-109;

e-print: http://arxiv.org/abs/math.GT/0509334

[Prz-5] J. H. Przytycki, Distributivity versus associativity in the homology theory of algebraic structures, Demonstratio Math., 44(4), December 2011, 821-867; e-print: http://front.math.ucdavis.edu/1109.4850

[Prz-6] J. H. Przytycki, Distributivity versus associativity in the homology theory of algebraic structures, in Mathematisches Forschungsinstitut Oberwolfach - Invariants in Low-Dimensional Topology and Knot Theory, Report No. 28/2012, 33-36.

[Prz-7] J. H. Przytycki, From Goeritz matrices to quasi-alternating links;

A Book Chapter (Chapter 9) in The Mathematics of Knots: Theory and Applications, M.Banagl, D.Vogel, editors; Springer-Verlag, 2011, pp. 257-315.

e-print: http://front.math.ucdavis.edu/0909.1118

[Prz-8] J. H. Przytycki, KNOTS: From combinatorics of knot diagrams to the combinatorial topology based on knots, Cambridge University Press, accepted for publication, to appear 2016, pp. 650 .

Chapter II, e-print: http://arxiv.org/abs/math/0703096

Chapter III: e-print: arXiv:1209.1592v1 [math.GT]

Chapter IV, e-print: arXiv:0909.1118v1 [math.GT]

Chapter V, e-print: http://arxiv.org/abs/math.GT/0601227 
Chapter VI, e-print: http://front.math.ucdavis.edu/1105.2238

Chapter IX, e-print: http://arxiv.org/abs/math.GT/0602264

Chapter X, e-print: http://arxiv.org/abs/math.GT/0512630.

[Pr-Pu-1] J. H. Przytycki, K. Putyra, Homology of distributive lattices, the Journal of homotopy and related structures, Volume 8(1), 2013, pages 35-65;

e-print: http://arxiv.org/abs/1111.4772

[Pr-Pu-2] J. H. Przytycki, K. Putyra, Degenerate distributive complex is degenerate, in preparation.

[Pr-Ro-1] J. H. Przytycki, W. Rosicki, The topological interpretation of the core group of a surface in $S^{4}$, Canad. Math. Bull., 45(1), 2002, pp. 131-137; e-print: http://arxiv.org/abs/math.GT/0403475

[Pr-Ro-2] J. H. Przytycki, W. Rosicki, Cocycle invariants of codimension 2embeddings of manifolds, Banach Center Publications, 103, 2014, to appear; e-print: arXiv:1310.3030 [math.GT]

[P-S] J. H. Przytycki, A. S. Sikora, Distributive products and their homology, Communications in Algebra, 42(3), 2014, 1258-1269; e-print: http://front.math.ucdavis.edu/1105.3700

[P-T-1] J. H. Przytycki, P. Traczyk, Invariants of links of Conway type, Kobe J. Math. 4, 1987, 115-139.

[P-T-2] J. H. Przytycki, P. Traczyk, Conway algebras and skein equivalence of links, Proc. Amer. Math. Soc., 100(4), 1987, 744-748.

[Put] K. Putyra, Cobordisms with chronologies and a generalisation of the Khovanov complex, Master degree Thesis. Jagiellonian University, Cracow, Poland, 2008; e-print: http://front.math.ucdavis.edu/1004.0889;

(see also 2-category of chronological cobordism and odd Khovanov homology, Banach Center Publications, 103, 2014, to appear).

[R-S] A. B. Romanowska and J. D. H. Smith, Modal Theory, Heldermann, Berlin 1985.

[R-Sa-1] C. Rourke, B. Sanderson, $\Delta$-sets. I. Homotopy theory, Quart. J. Math. Oxford Ser. (2), 22, 1971, 321-338.

[R-Sa-2] C. Rourke, B. Sanderson, A new classification of links and some calculations using it; e-print: arXiv:math/0006062 [math.GT]

[Schr] E. Schröder Über Algorithmen und Calculn, Archive der Math. und Phys., 5, 1887, 225-278.

[Ser] J-P. Serre, Lie algebras and Lie groups: Lectures given at Harvard University, 1964, W. A. Benjamin, Inc. New York-Amsterdam 1965 vi+247 pp.

[Si] A. S. Sikora, On Conway algebras and the Homflypt polynomial, J. Knot Theory Ramifications 6(6), 1997, 879-893.

[Slo] J. Słomińska, Coloured posets, graphs and homological algebra in functor categories, Bull. Pol. Acad. Sci.. Math., 60(3), 2012, 219-239.

[Spa] E. H. Spanier, Algebraic Topology, Springer-Verlag, 1966.

[Tak] M. Takasaki, Abstraction of symmetric transformation, (in Japanese) Tohoku Math. J. 49, 1942/3, 145-207; English translation is being prepared by S. Kamada.

[Tur] V. G. Turaev, The Yang-Baxter equation and invariants of links, Invent. Math., 92,1988, 527-553. 
[Tu-Wa] P. Turner, E. Wagner, The homology of digraphs as a generalisation of Hochschild homology, Journal of Algebra and its Applications, 11(2), 2011, 1250031, 13 pp.

[Va] M. Vaughan-Lee, The restricted Burnside problem, Second edition. London Mathematical Society Monographs. New Series, 8. The Clarendon Press, Oxford University Press, New York, 1993. xiv+256 pp.

[Wat] C. E. Watts, A homology theory for small categories, In: Proc. of the Conf. on categorical algebra, La Jolla, Canada 1965. (possibly the first paper defining generally homology of a small category with coefficients in a functor).

[Wei] Charles A. Weibel, An introduction to homological algebra, Cambridge studies in advanced mathematics 38, Cambridge University Press, 1994.

Department of Mathematics, The George Washington University, Washington, DC 20052

e-mail: przytyck@gwu.edu, University of Maryland College Park, and University of Gdańsk, Poland 